Portland State University

PDXScholar

Spring 6-8-2018

\title{
Latinas and Sexual Health: Correlates of Sexual Satisfaction
}

Christine Marie Velez

Portland State University

Follow this and additional works at: https://pdxscholar.library.pdx.edu/open_access_etds

Part of the Gender and Sexuality Commons, Social Work Commons, and the Women's Studies Commons

Let us know how access to this document benefits you.

\section{Recommended Citation}

Velez, Christine Marie, "Latinas and Sexual Health: Correlates of Sexual Satisfaction" (2018). Dissertations and Theses. Paper 4408.

https://doi.org/10.15760/etd.6292

This Dissertation is brought to you for free and open access. It has been accepted for inclusion in Dissertations and Theses by an authorized administrator of PDXScholar. Please contact us if we can make this document more accessible: pdxscholar@pdx.edu. 
Latinas and Sexual Health: Correlates of Sexual Satisfaction

by

Christine Marie Velez

A dissertation submitted in partial fulfillment of the requirements for the degree of

Doctor of Philosophy

in

Social Work and Social Research

Dissertation Committee:

Roberto Orellana, Chair

Gita Mehrotra

Maria Talbott

Christina Sun

Charles Klein

Portland State University

2018 
(C) 2018 Christine Marie Velez 


\begin{abstract}
Latinas/os are one of the fastest growing and most heterogeneous minority ethnic groups in the US. One in 5 women in the US are Latina; by 2060, it is projected that Latinas will compose $1 / 3$ of the female population. Latinas continue to experience disparities in sexual and reproductive health outcomes compared to non-Hispanic whites. While factors impacting undesirable consequences of sexual activity for Latinas have been well documented, Latinas' experiences with sexual satisfaction in the broader context of sexual health remains understudied, despite sexual satisfaction having been identified as an integral component of sexual health. A focus on positive sexual health outcomes for Latinas has the potential to challenge known stereotypes about Latina sexuality; specifically, those related to cultural constructs such as acculturation, machísmo and Marianísmo. Conversations about the positive aspects of sexuality and sexual wellbeing are largely absent from current social work literature, education and practice. Often times, cultural stereotypes about acculturation, machismo and marianísmo are perpetuated through risk-based approaches to understanding Latina sexuality. This study seeks to provide insight into factors correlated with sexual satisfaction for Latinas and to increase understanding of differences and similarities amongst Latina subgroups with respect to sexual satisfaction. This study is informed by Intersectionality and Latina Critical Race Theory; these theoretical approaches inform the research methodology and interpretation of findings by centering Latina identities and challenging stereotypes about Latina sexuality through a focus on positive aspects of sexual well-being.
\end{abstract}


This is a cross-sectional, secondary analysis of Wave IV data from the 2008 National Longitudinal Study of Adolescent Health (Add Health) study. Wave IV includes a sample of 287 adult Latina women, who identified as either Mexican (56.9\%), Chicana (6.7\%), Cuban (4.2\%), Puerto Rican (13.8\%), and/or Central American (11.7\%) or "other" $(12.9 \%)$, with some identifying as multi-racial. The mean age of participants is 28 years. ANOVA analysis identified no significant group differences amongst Latina subgroups with respect to sexual satisfaction. Bivariate correlations indicated statistically significant associations between sexual satisfaction and relationship satisfaction. After controlling for income, education and religion, multiple regression analyses showed that relationship satisfaction, number of vaginal sex partners, and frequency of sexual relations were significantly correlated with sexual satisfaction. The more frequent engagement in sexual activity, and the more sexual partners one has is correlated with higher levels of sexual satisfaction.

This study contributes to our knowledge of Latina sexual health, especially our understanding of factors that impact sexual satisfaction. For Latina women, health promotion programs should be designed to enhance interpersonal relationships that are based on mutual respect and care, utilizing culturally relevant approaches. Findings of this study challenge stereotypical cultural constructs related to acculturation, machísmo and marianísmo. This study shows that quality relationships built on trust, communication and love are strongly correlated with sexual satisfaction, which in turn should impact overall health. These findings support the recognition of positive aspects of sexuality as a critical site of intersectionality as Latinas of all ethnic groups in this sample report high levels of sexual satisfaction, as well as relationship satisfaction and 
support health promotion and intervention intended to support the cultivation and maintenance of meaningful relationships for Latinas. 


\section{DEDICATION}

I dedicate this dissertation to my parents, my late father Augustine F. Velez and my mother, Armida Orozco and my brother, Johann S. Velez. I could not have come this far without your love and support. I am so proud to be your daughter and sister. Besos y abrazos fuertes para siempre, les quiero tanto. 


\section{ACKNOWLEDGEMENTS}

I would like to acknowledge Scarlet and Rafael, mis hjos bonitos, my beautiful children...mi vida, mis amores, mi mundo...my life, my loves, my world...thank you for your patience as I complete this PhD journey. You may not have realized it, but your love, hugs, laughs, kisses and snuggles, have helped me make it through so many difficult times during this process. I'm so lucky to have you both to come home to everyday. You are my inspiration and hope. Mijos...les quiero tanto.

I would also like to acknowledge my husband Robert, I realize this hasn't been easy, thank you for your patience, forgiveness and love, I hope I have made you proud and that we have learned from our hardships and are on to a better and brighter future together as a family. Thank you for coming along on this journey with me, and for supporting me along the way. You are an incredible father to Scarlet and Rafael, thank you for carrying the load when I couldn't be present... we all know you are our rock. Te quiero mi amor para siempre.

Thank you to Dr. Roberto Orellana; thank you for your guidance and support, for your humor and for keeping me on my toes, for reminding me to never take myself too seriously. I have always enjoyed our conversations and have looked forward to our many meetings. You hold a special place in my heart, and I hope to pay this all forward with students I will be working with in the future.

I would also like to thank other members of my committee: Dr. Maria Talbott, Dr. Charles Klein, Dr. Christina Sun and especially Dr. gita mehrotra. gita, you have modeled so much for me about being a professor...thank you for helping me not only in this 
process but with landing a faculty positon. I know I have you to thank for helping my dream of being a social work faculty member come to fruition. I would also like to thank Dr. Bowen McBeath, I have so enjoyed getting to learn from you, thank you for all the support.

I would like to end with a big shout out to all my friends who have cried with me, laughed with me, worked out with me, shared meals, new jobs, disappointments and everything else. Beckie, Andre, Jared, Kevin, Joseph, Thuan, Sophie, and Danica, thank you, I love you all so much. Together, we got this! Thank you for believing in me! I believe in you! I'm so grateful to have you as peers and colleagues! You inspire me! And lastly, I want to acknowledge the amazing Latina, Chixana, Spanish, Black, African American women and other women of color whose work, scholarship and activism have ultimately made a project like this possible. I am forever grateful to you. I am thankful to all the women of color who stand beside me and who have come before me and who will come after me... who have stated that there is no one way to be Latina, that we are all in this together, and to never ever forget our ancestors or where we have come from, and to listen to the voices of the generations which come after us. ¡Si se puede! 


\section{TABLE OF CONTENTS}

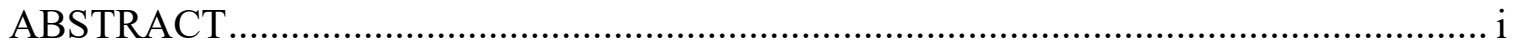

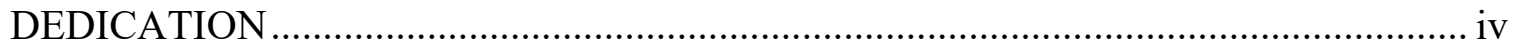

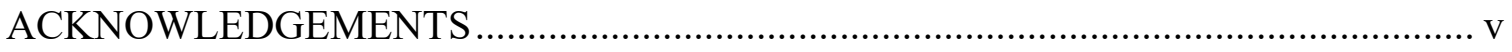

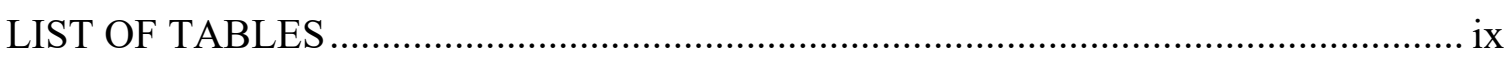

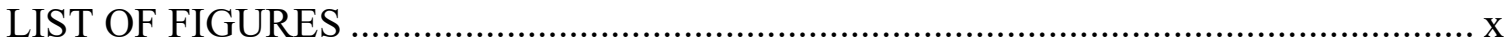

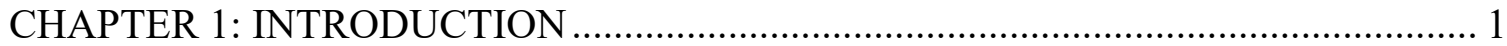

Goals and Objectives for Dissertation Research ......................................................... 6

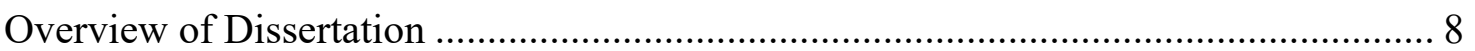

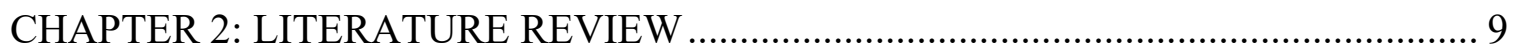

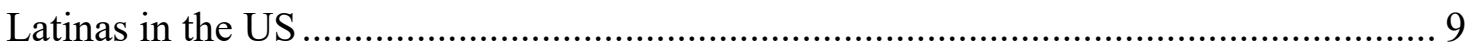

Historical Context: Social Work's Relationship with Immigrant Communities and

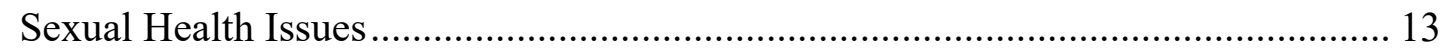

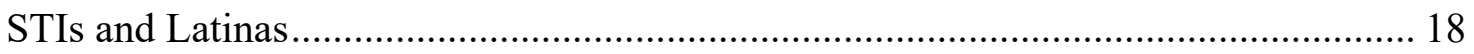

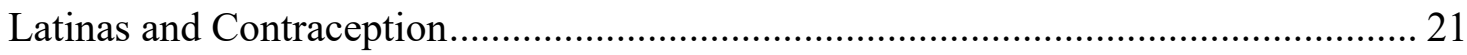

Sexual Health, Sexuality \& Sexual Well Being ........................................................ 23

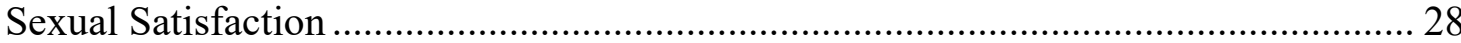

Unpacking Acculturation, Machísmo y Marianísmo …………………………………...... 31

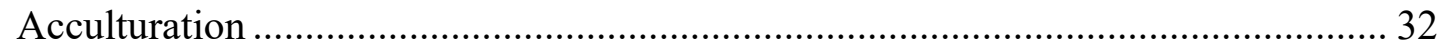

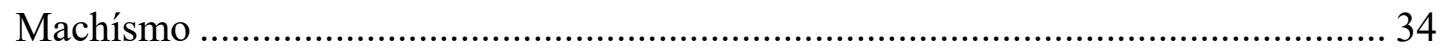

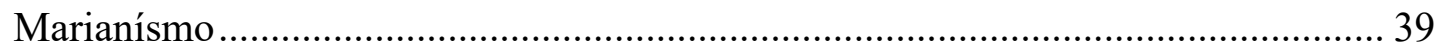

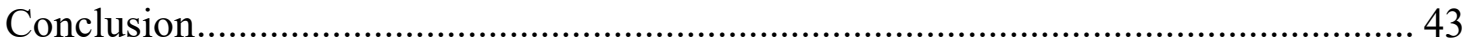

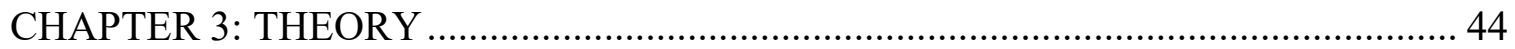

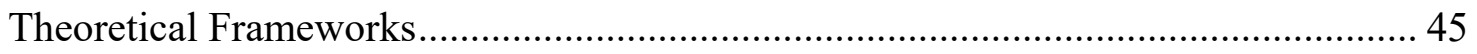

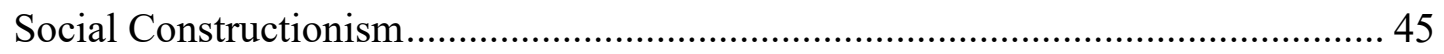

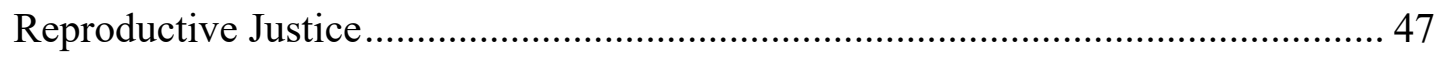

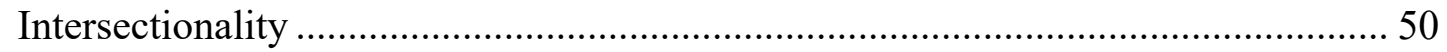

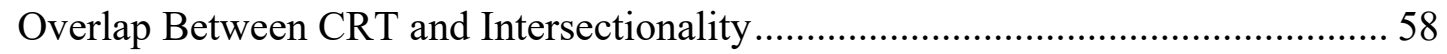

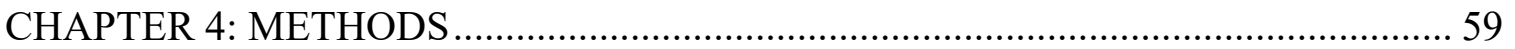

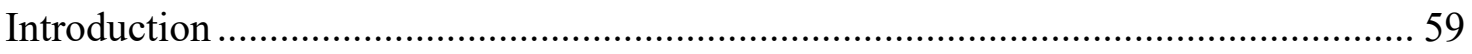

Study Design \& Add Health Background .................................................................. 59

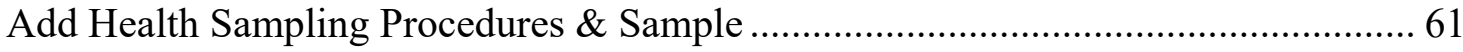




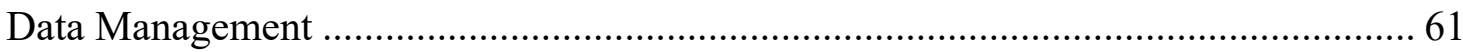

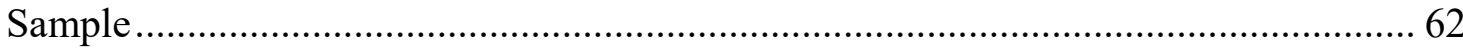

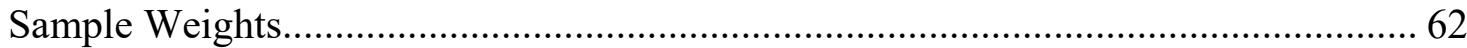

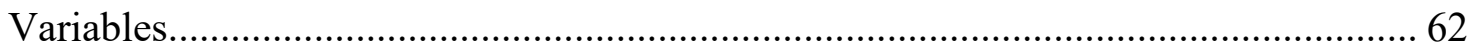

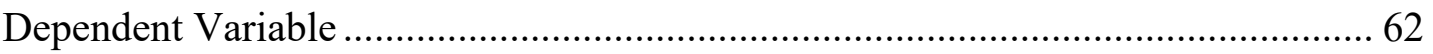

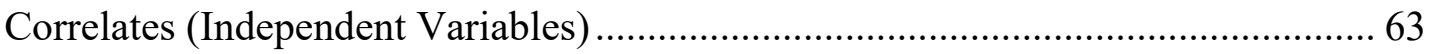

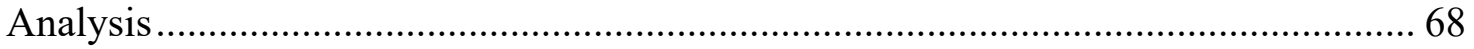

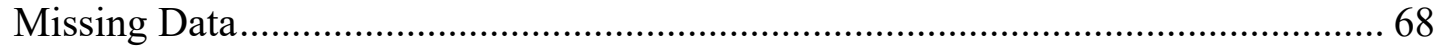

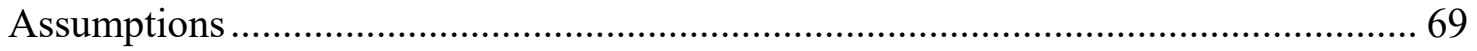

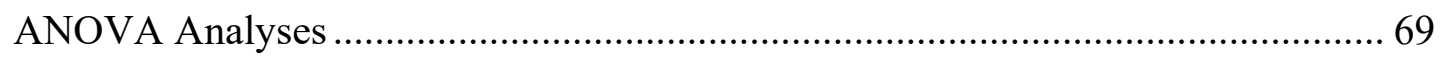

Bivariate Correlation Analyses............................................................................. 70

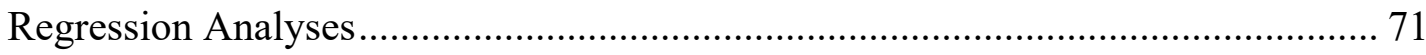

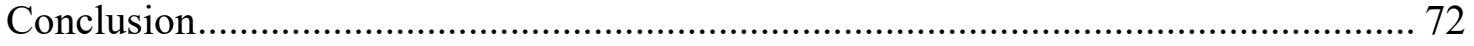

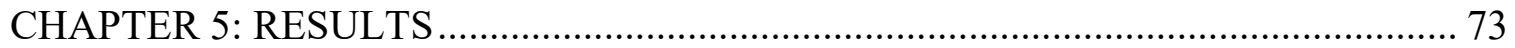

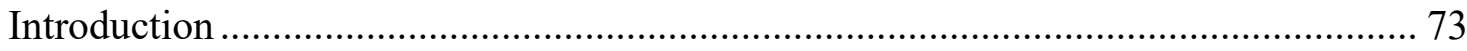

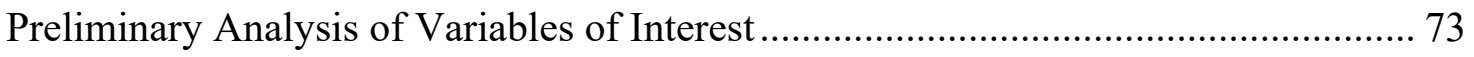

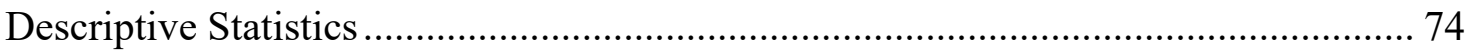

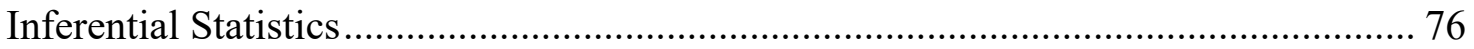

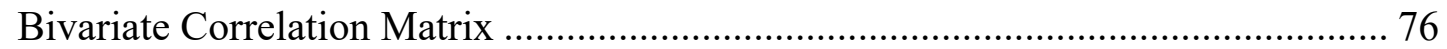

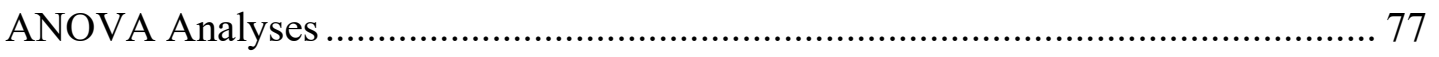

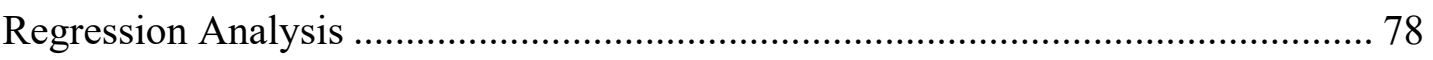

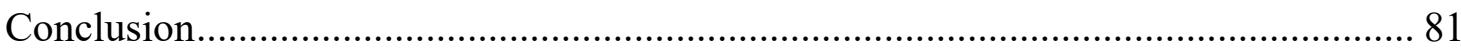

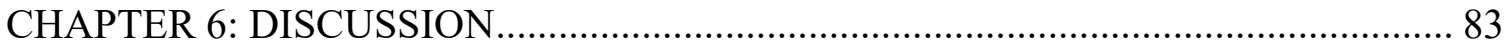

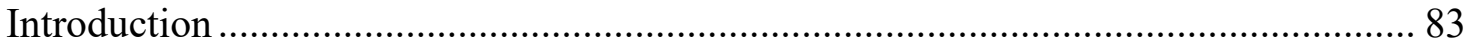

Meaningful Relationships, Sexual Satisfaction and Challenging Cultural Constructs . 83

Latinas, Sexual Satisfaction, Contraception and STIs ............................................... 88

Study Limitations and Directions for Future Research............................................... 90

Implications for Social Work Practice, Research and Education.................................. 92

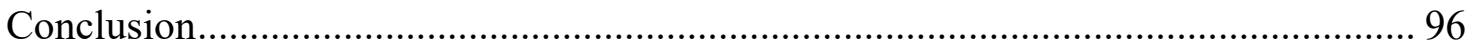

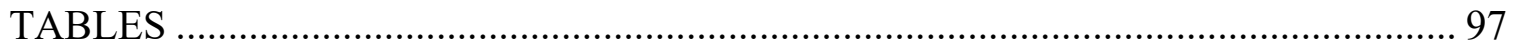

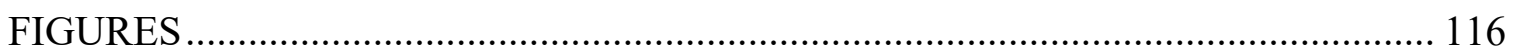

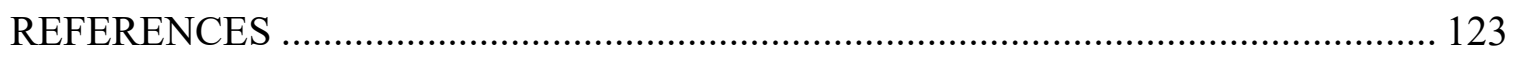

viii 


\section{LIST OF TABLES}

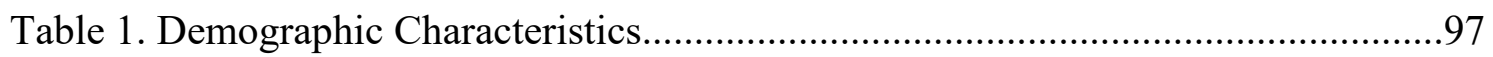

Table 2. Correlations between Sexual Satisfaction and Socioeconomic Factors.............105

Table 3. Correlations between Sexual Satisfaction and Sexual Behaviors.......................106

Table 4. Correlations between Sexual Satisfaction and Relationship Characteristics.....108

Table 5. One-way Analysis of Variance of Sexual Satisfaction in Latina Women by Subgroup.

Table 6. One-way Analysis of Variance of Number of Vaginal Sex Partners in Latina

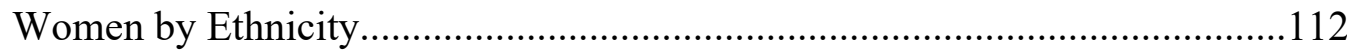

Table 7. Regression of Time Involved with Current Partner Controlling for Income and

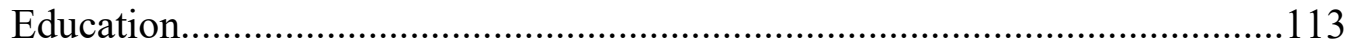

Table 8. Regression of Relationship Satisfaction Controlling for Income and

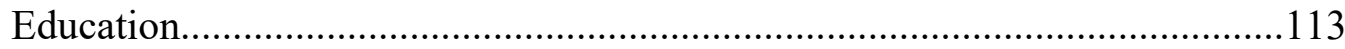

Table 9. Regression of Number of Sexual Partners Controlling for Income, Education, and Religion.

Table 10. Regression of Frequency of Sex Controlling for Income, Education, and Religion

Table 11. Regression of Sexual Satisfaction on Individual Relationship Variables on for all Latinas.

Table 12. Regression of Sexual Satisfaction on Number of Vaginal Sex Partners, Frequency of Sexual Activity and Time Involved with Current Partner for all Latinas. 


\section{LIST OF FIGURES}

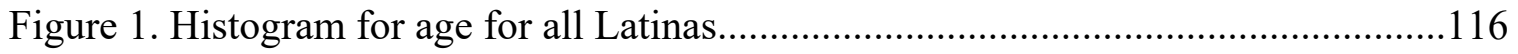

Figure 2. Histogram for household income for all Latinas.........................................117

Figure 3. Histogram for number of vaginal partners ever had for all Latina group........118

Figure 4. Pie chart for Education for Latinas.............................................................. 119

Figure 5. Means plot of sexual satisfaction and Latina subgroups..............................120

Figure 6. Means plot of number of vaginal sex partners among Latina subgroups.........121

Figure 7. Stem and Leaf Plot for Number of Vaginal Sex Partners for Puerto Rican Latina

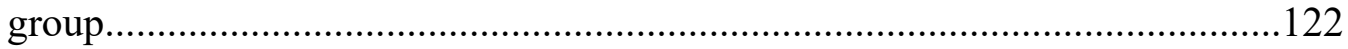




\section{CHAPTER 1: INTRODUCTION}

Discussion of Latina sexual health and sexuality remains highly politicized and controversial in our current political and social climate. Many dimensions of Latina sexual health remain unexplored in research; stereotypes and misconceptions about Latina sexuality and sexual health continue to permeate research and scholarship on this topic (Asencio \& Acosta, 2010; Arrevalo \& Amaro, 2010; Arrizón, 2008; Garcia, 2012; González-López \& Vidal-Ortiz, 2008). It has been well established that Latinas have poorer sexual health outcomes than their white peers with regards to HIV, sexually transmitted infections (STIs) and cervical cancer (CDC, 2016; Cashman et al., 2011). However, key domains of sexual health such as sexual pleasure, satisfaction and desire remain understudied in Latina populations. Furthermore, few studies have applied intersectional and critical theorizing to quantitative designs; perspectives which show promise in further illuminating issues with individual and structural level health disparities (Warner \& Brown, 2011). These theoretical perspectives align with social work's values and approaches to social justice focused research agendas. Intersectionality and critical theories are useful tools in sexual health research which have the potential to enhance our understanding of the micro, mezzo and macro underpinnings impacting sexual health in immigrant communities and communities of color.

In this introduction, I will briefly provide some context and background of Latinas living in the United States (US) and identify sexual health needs of this population. This research aims to investigate correlates of sexual satisfaction for Latina women and explore variables impacting sexual health outcomes. I will identify my goals 
and objectives for dissertation research and will provide an overview of this dissertation, including research questions guiding design and analysis.

I want to be clear that this study and the scholarship informing it builds upon the work of other researchers and academics, mostly women of color, who have paved the way for a critical, and sex positive approach to engaging with Latina sexuality and sexual health. Gloria Anzaldúa, Cherie Moraga, Marisol Asencio, Patricia Hill Collins, are just a few scholars whose work have made a project like this possible. Although these scholars are not explicitly situated within the field of social work; building off of their scholarship and those of others demonstrates the utility of interdisciplinary work in the field of sexuality and sexual health. These writers have offered a counter dialogue to essentialist conceptualizations of Latina sexuality and this project, nested in social work, continues this work.

According to the US Census Bureau (2015) and the Pew Research Center (2016), the Latina/o population reached 57 million in 2015, representing approximately $17.8 \%$ of the overall US population. The Latina/o population is $63.4 \%$ Mexican origin, $9.5 \%$ Puerto Rican, 3.7\% Cuban, 3.3\% Dominican, 2.4\% Guatemalan and the remainder are from other Central and South American countries, indicative of the rich diversity present among Latinas/os (Gandara et al., 2015; Krogstad, 2016; US Census, 2016). In 2015, the following states had 1 million or more Hispanic/Latina/o residents: Arizona, California, Colorado, Florida, Illinois, New Jersey, New Mexico, New York and Texas. The median household income for Hispanic/Latina/o households in 2015 was \$45,150. Approximately $21.4 \%$ of Hispanic/Latina/o households live at or below the federal poverty line and about 16.2\% lack health insurance (Gandara et al., 2015; Krogstad, 
2016; Planned Parenthood, 2013; US Census, 2016). In 2013 the US Census Bureau reported that among Hispanics, the national poverty rate ranged from $16.2 \%$ amongst Cubans to 26.3\% for Dominicans (Macartney, Bishaw \& Fontenot, 2013). It is clear from these statistics that attention to health issues impacting Latinas/os should be of pressing concern to researchers and academics, and especially social workers. The Latina/o condition in particular should be a focus for social workers across the US, because based on these aforementioned statistics alone, it is highly likely that social workers will engage with Latinas/os service users in some capacity.

One is 5 women in the US are Hispanic/Latina; in 2011, one quarter of all births in the US were born to Latina women (Gándara et al., 2015). Despite considerable educational gains in the past several years, Latinas continue to graduate high school at a lower rate than any other major ethnic subgroup and are the least likely of any other racial/ethnic group to complete a college degree (Gándara et al., 2015).

Regarding reproductive and sexual health outcomes, Latinas experience higher rates of the human papillomavirus (HPV) than white women and are twice as likely to die from HPV related cervical cancer than their white peers (CDC, 2016). Latina women represent $17 \%$ of new AIDS diagnoses among women, and experience unintended pregnancy at twice the rate of white women (Jackson, 2013; Gándara et al., 2015; Planned Parenthood, 2013).

Researchers have identified several individual risk factors and health behaviors associated with poorer health outcomes for Latina women. Much of this literature utilizes risk frameworks which often fail to acknowledge pleasure, desire, sexual orientation, sexual fluidity and the sensual desires of Latina women (Afable-Munsuz \& Brindis, 
2006) despite pockets of scholars engaging in sex positive scholarship. Many frameworks present in social work approach the Latina body as a site of "risk" and in need of intervention. Many studies focus on the potential harm associated with sexual activity (such as unintended pregnancy) and are focused on the ways in which Latinas can mitigate or avoid these harms altogether (Garcés-Palacio et al., 2008; Guerra-Reyes et al., 2017; Martinez et al., 2014). The presence of risk frameworks in addressing sexual health disparities is by no means specific to Latina women, as they are applied liberally to many other marginalized groups. For example, risk frameworks are often applied to research with men who have sex with men (MSM) and gay men; topics which are outside the scope of this particular project but nonetheless provide evidence of the wide application of risk frameworks to many marginalized groups. I contend that there is a place for risk frameworks, as much as there also needs to be space for addressing sex and sexuality from sex positive frameworks in marginalized communities.

Some scholarship on sexual health and Latinas has extended beyond focusing solely on STIs and HIV, and other undesirable consequences of sexual activity to focus on other dimensions of sexual well-being (Higgins et al. (2011); Sandfort \& Erhardt, 2004), an effort supported and catalyzed by the World Health Organization (WHO) and their 2002 definition of sexual health which I will explore in more detail later. Higgins et al. (2011) assert that the public health arena has recently been more focused on positive approaches to the study of sexual health and sexuality, although much of this work is not necessarily Latina specific. Cheng et al.'s (2014) study for example, acknowledge other dimensions of sexual health such as sexual self-efficacy and access to comprehensive sex education as critical components to healthy adult sexual functioning, although Cheng et 
al. did not focus exclusively on Latinas, Latinas were included in their sample. Lagana \& Maciel's (2010) qualitative investigation of sexual desire amongst older MexicanAmerican women found that the majority of participants reported their sexual needs as being unmet and demonstrated the need for normalized discussions of sexuality between patients and providers. In their quantitative study of the psychosocial factors impacting sexual satisfaction in a sample of Chilean women, Barrientos and Páez (2006) found that high SES was correlated with sexual satisfaction amongst participants. Although few, these studies demonstrate that researchers and academics are addressing positive dimensions of sexual health and behavior (Higgins et al., 2011), and set a precedent for exploring the research questions in this study.

This increased attention to dialogue and engagement with sexuality rights discourses are evidence of the growing attention on how to best leverage positive sexual health behaviors to promote sexual health and well-being (Higgins et al., 2011). It is urgently necessary to address issues impacting Latina sexual health in an effort to reduce health disparities impacting this heterogeneous group of women. Attention to health issues impacting Latinx communities is a pressing need not only because the Latinx community is a fast growing demographic, but also because they are targets of xenophobic policies from past and current presidential administrations.

I am currently aware of only one other study (Barrientos and Páez (2006)) which investigates sexual satisfaction in a sample of Latina women. However, Barrientos and Páez's (2006) study was conducted in Chile, with a sample of Latina women who all identified as Chilean. I am unaware of a nationally representative US study investigating sexual satisfaction in a sample of Latina women. Intersectional and critical theories have 
been widely embraced in social work education, research and practice and are compatible with social work values and ethics (NASW, 2015) and have the potential to inform health promotion work. These theoretical perspectives also provide useful guidance on challenging pervasive stereotypes about Latina sex and sexuality which can in turn inform culturally relevant and strengths based social work practice, education, and research.

Goals and Objectives for Dissertation Research

The overarching goal of this study is to advance understanding of sexual healthspecifically sexual satisfaction, amongst a nationally representative sample of Latinas in the US. I am applying intersectionality and critical race theory (CRT), specifically Latina/o Critical Race Theory (LatCrit) to formulation of the research questions as well as to the plan for analysis and interpretation of findings for this project. Using the National Longitudinal Study of Adolescent Health (Add Health), I conducted a secondary data analysis examining the extent to which sexual satisfaction is associated with sexual behavior variables, relationship variables and/or SES/structural variables, and to assess differences based on Latina ethnic subgroup variability. The following research questions address these objectives and guide research design:

1. What are the correlates of sexual satisfaction among Latinas?

2. What is the relationship between sexual satisfaction and contraception amongst Latinas?

3. What is the relationship between sexual satisfaction and STI prevalence amongst Latinas? 
4. Are there any differences in sexual satisfaction by Latina sub-groups?

The first step in aligning with an intersectional and critical framework is for me, as a researcher, to engage reflexively during all stages of the research process (Hancock, 2016). The impact of lack of reflexive engagement can "render the researcher invisible, disguises power processes, distorts and misrepresents actual words, perspectives and experiences of participants and obscures the contested nature of science and knowledge" (Leotti \& Muthanna, 2014, p.180). As such, and consistent with social work's emphasis on reflexivity in praxis, I engage reflexively in this quantitative study. My social work experience is largely based on working with families and individuals in non-profit settings in upstate New York. Specifically, I have worked for organizations primarily servicing Latinas/os and Hispanic populations. I worked in a cancer hospital as a social worker (mostly working with Spanish speaking families), as a refugee resettlement case manager and as a case manager, in a program for Latinas/Hispanic women who were survivors of intimate partner violence, and their children. It was through these experiences that I not only learned about policy impacting various aspects of sexual health, such as helping women navigate Medicaid and getting access to reproductive health care, but also about cultural strengths of women and communities and strategies they employed to get their needs met.

My educational experiences as a graduate student in a school of social work introduced me to concepts such as intersectionality, critical theories and reproductive justice. I myself identify as a Latina, Hispanic, first generation, middle-class, cis-gender female. I am bilingual in English and Spanish, and am also a mother of two children, and have personal experiences accessing reproductive health care myself. The combination of 
these work experiences combined with my own social location influence and impact my view of the issue of sexual health and Latinas, and may impact how I interpret findings and results. I personally see the cultural stereotyping of Latinas as harmful, as well as assumptions that we are all a homogenous group. I certainly recognize how the intersection of race, class, gender, social status impacts my world view and conceptualization of the strengths and limitations of social work practice and research.

\section{Overview of Dissertation}

Chapter two begins with a review of relevant literature, where I provide more context and background as to Latinas living in the US as well as an overview of the historical context through which social work and immigrant sexual health issues intersect and exist. I will then define terms related to the concept of sexual health, and provide an overview of STIs, contraception and Latina populations. I also unpack some cultural constructs present in the literature on Latinas and sexual health such as acculturation, machísmo and marianísmo. Chapter three discusses and identifies social constructionism, reproductive justice, intersectionality and Latina/o Critical Race Theory (LatCrit)c as theoretical frameworks guiding design, analysis and interpretation of findings. Chapter four describes the research methodology and analysis plan as it relates to chapters two and three. Chapter 5 is the discussion section which also describes the study's limitations as well as implications for social work research, education and practice. I end this dissertation with a brief conclusion in Chapter 6, including reflections for future work. 


\section{CHAPTER 2: LITERATURE REVIEW}

In this literature review I will first provide a brief historical background of Latinas in the US, an approach consistent with intersectionality and LatCrit. I will then identify and define various definitions associated with sexual health and sexual well-being. I will review the literature on Latinas, STIs and contraception and lastly I will unpack three cultural constructs present in the literature on Latinas and sexual health: acculturation, machísmo, and marianísmo. I recognize that there are other constructs and concepts related to the larger topic on Latinas and sexual health. I have chosen to focus on these as I believe they are relevant to my research questions and analysis of this data. I feel it is important to provide some background into the literature on STIs, contraception and Latinas because of my research questions about contraceptive preferences of women in the sample. As such, some of the relevant literature points to acculturation, machísmo and marianísmo as possible explanations for high STI rates and low contraceptive utilization amongst Latinas.

Latinas in the US

Individuals of Hispanic and/or Latin American descent represent a growing demographic in the US (Pew Research Center, 2017). A basic historical understanding of the colonization of Latin America is critical to grasping the Latina condition today. The impacts of Spanish colonialism and the African slave trade were profound, and we cannot engage with the Latina experience today without acknowledging and grappling with a horrific and traumatic history. Critical Race Theory (CRT), which I will explore in greater detail in the following chapter, supports acknowledging historical perspectives of 
issues impacting people of color today. CRT and LatCrit encourage engagement with historical and contextual processes to situate a given social issue impacting communities of color.

Consistent with LatCrit's emphasis on the historical underpinnings shaping our current reality, I will briefly discuss the colonized history of Latina sexuality (Arrizón, 2008). One component of the Spanish agenda to conquer the Americas included the search for wealth in the form of silver and gold. In the process of amassing resources to bring back to Spain, Spaniards also conquered people and land, a tactic justified through a Catholic doctrine of "saving" indigenous peoples and cultures from their "barbaric and primitive ways," with Christianity (Gutierrez, 2010). The first Spaniards to come to Latin America were male, young and single, and eager to make a fortune and conquer. Gutierrez (2010) suggests this colonization of the Americas as a sexual conquest in and of itself, and the history of Latina/o sexuality begins with this conquest in a specific date and time. Latinas/os are products of these historical moments, which shape our discourses around sex and sexuality and what sexual health means, especially when rape and assault are such profound components of that history. Undoubtedly, the Catholic Church, and other forms of Christianity, such as Evangelism, and colonialism have had tremendous impact over sexuality discourses throughout history and through the present day. The Catholic church sanctioned and even encouraged the rape and assault of indigenous peoples (Gutierrez, 2010). Christian religions are traditionally "patriarchal institutions which have subordinated and oppressed women" and thus have strict rules about sex (which should only occur in the context of procreation, and for male satisfaction) and contraception (Catholicism explicitly frowns upon its use), as well as the role of women 
in the family (Padilla, 2011, p. 503). The complex and dynamic relationship between Christianity and Latina sexuality is beyond the scope of this study but necessary to briefly mention as it connects to past and present day forms of colonialism experienced by Latinx communities.

Latinx and/or Hispanic identified individuals represent the largest and fastest growing demographic in the United States (Buhi et al., 2014; Cheng et al., 2014; Rodriguez, 2008; Zavella \& Castenada, 2005). However, they are not a homogenous group. Multiple factors shape Latina identity, and this section will focus on a few of them. Usually in research, it is common practice to lump all subgroups together into categories of "Latina/o" and/or "Hispanic." Generally speaking, the term "Hispanic" refers to people of Spanish descent, that is, from Spain. The language we use to describe our individual Latina identities is complicated, and politically laden. For example, Gutierrez (2010) calls on scholars to resist and challenge the proclamation of the mestizaje historical account which "celebrates the extensive biological mixing" of Spaniards with Indigenous peoples (p.6). Instead, we must grapple with a history fraught with rape and violence and reject accounts that suggests that the biological mixing of indigenous and European persons was consensual and a cause for celebration. This complex, violent history complicates the notion of identity and how Latinas/os and Hispanics might identify. As such, not all Latina/o and/or Latin American people identify, if at all, as Hispanic, although many do (Rodriguez, 2008). Latina/o is a term generally used to describe people of Latin American descent and who speak Spanish (although not always) and encompasses multiple identities (Gloria \& Castellanos, 2013). Sandra Cisneros has stated, “'Hispanic' is English for a person of Latino origin who 
wants to be accepted by the white status quo. 'Latino' is the word we have always used for ourselves." Lumping all these groups together, although common in research and other areas of academic inquiry, can be seen by some as problematic as it does little to explore the uniting and dividing aspects of Latina/o identity (Castro-Salazar \& Bagley, 2012; Rodriguez, 2008) but is often necessary for political and community mobilization. We must also recognize that many Latinas and/or Hispanic women may also identify as Black and/or Afro-Latina, hence, the importance of intersectional lenses through which to underscore the various and different social locations and racial identities we can hold at the same time. The term "Latina" is a gendered term which interlaces gender with ethnicity (Gloria \& Castellanos, 2013). Additionally, just because someone might identify as Latina, Hispanic, or even Black- they may or may not also identify as a person of color. The political historical differences related to politics and colonialism differ from one group to the next and exploring these are necessary and important (Oboler, 2011) especially in connection to health.

Numerous differences exist amongst these subgroups though very often data collection tools fail to address the unique diversity within larger "Latina/o and/or Hispanic" groups. To be clear, we are not dealing with one set of national values, ideals or norms (Gutierrez, 2010). There are significant social, cultural and even historical differences between all of these Latina/o subgroups (Gutierrez, 2010; Rodriguez, 2008). In addition, many indigenous groups (Tarahumaras, Tarascos, Zapotecs, etc) also often get classified as "Hispanic" and/or Latina/o when perhaps they themselves might not identify as such (Castro-Salazar \& Bagley, 2012). Additionally, as Castro-Salazar \& Bagley (2012) point out, there are many Filipinos, Mayans, and Quechuas who have 
Spanish surnames imposed upon them because of colonialization. Because many studies continue to look at the larger "Latina/o" and/or "Hispanic" categories, the following literature review will follow those two categories specifically, while also recognizing the robust body of work that addresses variability within these subgroups.

Historical Context: Social Work's Relationship with Immigrant Communities and Sexual Health Issues

The close examination of reproductive and sexual health issues for women is important for social work practice, education and research especially considering that globally and locally women continue to face many barriers to reproductive health care (Winter et al., 2016; Wright, 2015) which are increasing in the US today. In order to be able to discuss current implications of this study upon the field of social work, it is necessary to briefly address social work's complicated history with immigrants, women of color, sexual health and Mexicans in particular. I focus on Mexicans in particular because Mexicans make up one of the largest Latina subgroups (Gandara et al., 2015) and because they also represent the largest Latina group in this sample for this study. Jane Addams famously discussed the "vexed problem of immigration," (Addams, 1909) and helped set the stage for a number of "culturally inappropriate policies and services that have maintained prevailing injustices" which were to follow (Resich, 2008, p.789). Although social work as a discipline has had many positive societal impacts, consistent with a critical theory informed approach, it must also be acknowledged that social work has a history denying legitimacy and agency to people of color and immigrant groups in favor of paternalistic approaches to service provision (Park \& 
Kemp, 2006; Resich, 2008). For example, social work played a large role in placing Japanese individuals and families in internment camps during World War II (Park, 2008), in removing Native American children from their families into boarding schools, and in advocating for putting sex workers, at the time referred to as "prostitutes" in jails (Krumer, Berkovitz-Romano \& Komem, 2015). Social workers at the time took part in these atrocities through adopting a moral authority that as a discipline, we knew what was best for these "othered" groups. This acknowledgement is part of social work history must be acknowledged; this is consistent with critical race theorists, intersectional and reproductive perspectives which are further explored in chapter 3.

Resich (2007) outlines four historical approaches of American social workers in addressing social justice: assimilation, separatism, cultural pluralism and color blindness. All are seriously damaging and oppressive attitudes through which to understand and respond accordingly to various needs of Latinas and immigrant groups in general. Although this is an important part of the history of social work which illuminates the professions' tension with immigrants and people of color today, certainly much has changed since the early days of professional social work practice. However, racial microaggressions and paternalism on the part of the social worker towards people of color have not as of yet been completely eliminated. The push for greater cultural relevancy in social work practice, along with a person in environment and strengths based perspectives are evidence that the field as a whole is rejecting the antiquated social work perspectives related to assimilation, separatism, cultural pluralism and color blindness in favor of an anti-oppressive, anti-racist approach. A richer understanding of understudied elements of sexual health for Latinas has the potential to inform future social workers as to the 
nuances of sexual health, race, and health promotion in an effort to reduce the pathologizing or essentializing of cultural groups consistent with social justice and antioppressive values outlined in our code of ethics (NASW, 2015).

Social work also has a historically complicated relationship with Mexicans in particular; as pockets of social work have supported understanding Mexican immigration as a "social problem" worth attending to. McWilliams (2011) draws attention to social work's role in the formulation of the "Mexican problem," beginning in 1924 with the passage of the Immigration Act which outlined social problems caused as a direct result of immigration from the southern border. McWilliams (2011) identifies the many ways in which social work agencies benefitted from the "social problem" that was Mexican immigration in the creation of a number of social programs tailored towards "fixing" this problem:

The whole apparatus of immigrant-aid social work, with morose preoccupation with consequences rather than causes, was therefore transferred to Mexican immigration with little realization that this immigration might not be, in all respects, identical with European immigration (p .149).

McWilliams is alluding to early social work efforts where "most white social workers did not define social justice in terms of full social equality," and whereby social workers were unable to distinguish between different needs of various immigrant groups in favor of lumping them all together (Resich, 2008, p.789). This reflects the historical and I would argue present day tension between "providing services based on mutuality and collectivism and those rooted in individualism and the preservation of professional status" (Reisch, 2008, p.789). 
McWilliams (2011) also asserts that social workers were tasked in the past to collect data on the "problems" of Mexican immigration where "data was consistently interpreted in terms of what it revealed about the inadequacies and weaknesses of the Mexican character," where the data "proved that Mexicans lacked leadership, discipline, organization" etc. (p. 149). In a sense, this parallels a version of what we see now with risk frameworks about health and wellbeing by focusing on the "risky behavior" of Latinas and elements of Latina culture which place Latinas in a state of "risk" instead of better understanding the micro, mezzo and macro underpinnings which exacerbate health disparities for this population.

This study addresses Latina sexuality not as a "problem" or "risk factor" but as something yet to be well understood by researchers. Past social work approaches have sought to control the reproductive destinies of women of color which often shame the expression of Latina sexuality by denying "legitimacy and agency" to Latina/o groups to decide the needs of their own communities (Asencio, 2010). This occurs when social work takes on the savior role of "we know best." Social work's long affiliation with Christianity, and in particular, the Catholic church and Catholic charities (Resich, 2008) exemplify the ways in which social work has been complicit in the regulation of bodies of color. Catholic and Christian doctrine, with its powerful influence, has set clear guidelines regarding contraception, abortion and reproduction of women's bodies. Rarely is pleasure and satisfaction present in the discussion of sex and sexuality in these contexts. Sexual activity occurs in the context of procreation only under traditional Christian and Catholic traditions, where women are often actively discouraged from enjoying sex (Padilla, 2011). Attempting to identify differences in sexual satisfaction, 
contraceptive use, and STI prevalence amongst various Latina subgroups has the potential to illuminate certain differences or similarities and might provide guidance on sexual health promotion and help to encourage social workers to better understand the needs of various subgroups of Latinas without presupposing a superior moral compass over this ethnic subgroup of Latinas.

The landscape around sexuality studies within social work is changing, albeit at a slower pace than perhaps other disciplines. The current focus on anti-oppressive, antiracist and strengths based praxis within social work has opened the door to sex positive and culturally relevant social work scholarship (Bay-Cheng, 2013). Despite some gains in this arena, scholars allude to a missing discourse in social work around sexuality, as the profession continues to lean heavily on deficit models of sexuality in education, research and practice (Bay-Cheng, 2013; Dodd \& Tolman, 2017; McCave, Shepard, \& Winter, 2014). Formal mechanisms to support the study of human sexuality from sex positive frameworks such as textbooks, journals and national conferences are still rather limited for social workers (McCave, Shepard, \& Winter, 2014). Dodd \& Tolman (2017) have argued that because of this, social work is missing a critical opportunity to contribute to the larger conversation around healthy sexuality "that acknowledges desire and sexual satisfaction across the spectrum, including among marginalized and oppressed groups" (p.227). Some of the recent positive social work contributions to the sexuality discourse are seen in scholarship that addresses resiliency and social networks in LGBT populations (Elze, 2003; Giunta \& Rowan, 2015). Social work scholars and educators are also quite visible when it comes to unpacking dominant narratives around heteronormativity as well as in the attentiveness to sexuality discourses in people with 
disabilities and chronic health problems (Dodd \& Tolman, 2017). As such, having a journal or national conference for and by social workers to address human sexuality across the lifespan is much needed in the field.

Social work must address how data is used and disseminated, but must also ensure that our interpretations do not perpetuate harmful stereotypes which further oppress and marginalize groups of people. Latina/o culture is often susceptible to stereotyping and over simplification (Asencio \& Acosta, 2010). Certainly, popular culture has advanced narratives of the overly sexualized Latina or submissive Latina. Stereotyping of any group, albeit often unintentional, is problematic as it impedes our ability to work within culturally relevant, anti-oppressive and strengths based frameworks and models to address broader social inequities, which often perpetuate and maintain health disparities.

STIs and Latinas

Despite continued technological advances in medicine and healthcare, racial and ethnic minorities in the US continue to experience poorer health outcomes than their white counterparts (Keefe, 2010; Rogers \& Kelly, 2011). Latinas, a heterogeneous group, are disproportionately impacted by sexually transmitted diseases (STIs) as well as unintended pregnancy (Buhi et al., 2014). According to CDC analysis of 2010 data, rates of Gonorrhea infections amongst Latina/Hispanic groups are 2.2 times those of nonHispanic whites (CDC, 2016). The rate of chlamydia infections of Latinas/os is 3 times that of their white counterparts (CDC, 2016). Syphilis rates are 3.1 higher in Latinas/Hispanic women than whites. STIs would be expected to have a negative influence over sexual agency, desire, pleasure and satisfaction. In addition, protecting 
oneself against the threat of STIs could compromise one's perceived right to sexual pleasure. Two studies have indicated that participants reported less pleasure when using condoms (Widman et al., 2014). Similarly, Tanner \& Hensel (2016) found that amongst participants in their sample, higher sexual satisfaction was associated with more frequent sexual activity and less condom use. However, both of these aforementioned studies included predominantly white samples.

According to CDC analysis of 2010 data, Latinas/Hispanics make up 17\% of the population and account for $21 \%$ of all HIV diagnoses. As 2015 CDC report on HIV diagnoses and prevalence rates amongst Latinas/os living in the US found that amongst HIV diagnoses in Latina/Hispanic women, $86 \%$ was attributed to heterosexual sexual activity and $5 \%$ was related to injection drug use. Per the CDC, the HIV diagnosis rate among Latina/Hispanic women in 2015 was more than three times that of white women. Of all HIV diagnoses in Latinas, $48 \%$ were born in the United States, $20 \%$ Mexico, and $15 \%$ Puerto Rico.

Latinas continue to disproportionately bear the burden of HPV and cervical cancer in the US, more so than any other racial/ethnic group, with low-income Latinas at highest risk (D'Orazio et al., 2014; Fernandez et al., 2009; Garcini et al., 2015; Reimer, Houlihan, Gerrard, Deer, \& Lund, 2013; Seal et al., 2012; Yeganeh, Curtis, \& Kuo, 2010). HPV related cervical cancer is the second most common cancer for Latinas living in the US (CDC, 2016). In the US as well as other "developed" countries, most cases of cervical cancer are caused by HPV despite the fact that cervical cancer remains a preventable disease (Garcini et al., 2015). HPV and cervical cancer offer an opportunity to better understand how sociocultural factors shape risk and experience (D'Orazio, 
2014). Current research identifies a number of factors which contribute to elevated prevalence and incidence rates of HPV in Latina populations such as: lack of access to health insurance \& preventive care, and lack of transportation, inability to financially cover health care, etc.

Researchers studying the relationships between sexuality, sexual and reproductive health, and illness have affirmed that "sexually transmitted infections are dynamic and complex sociocultural historical processes characterized by inequalities of power and gender relations that actively structure and transform people's sexual meanings and practices" (Quevedo-Gomez, Krumeich, Abadia-Barrero, Pastrana-Salcedo, \& Van Den Borne, 2012). Despite the complexity of STIs and the sociocultural, historic, structural factors that lead to health disparities among Latinas, and high rates of STIs in particular, research on this topic continues to focus on individual level factors leading to high disease prevalence. Certainly, it is important and valuable to have a working knowledge of individual behavior impacting health, however, only focusing on individual behavior may obscure other factors perpetuating health disparities. Relating to social work specifically, some scholars have suggested that this focus is consistent with neoliberalism influences on social work practice, which tend to be preoccupied with individual behavior and resiliency (Reisch, 2013). Intersectionality, with its acknowledgement that multiple factors together lead to certain outcomes, encourages looking at all factors which may impact a given phenomenon, in this case, high rates of STIs.

Researchers have identified several structural reasons for these disparities. Lack of health insurance access and socio-economic issues such as lack of resources, language 
barriers, and transportation impact sexual health disparities (Seal, Garces-Palacio, Halanych \& Scarini, 2012). Rising xenophobia combined with discrimination towards Latin, Central and South Americans, combined with a lack of sex and sexuality education which departs from an abstinence only framework have also been identified as contributing factors to these sexual health disparities (Arevalo \& Amaro, 2010; Planned Parenthood, 2013). High incarceration rates of Latinos/as and low representation in institutions of higher learning have also been identified as potential contributors to sexual health disparities. Lack of access to quality sexual health education is a primary driver for sexual health disparities. Additionally, there is a shortage of social services to help marginalized groups navigate our complex health care system. Anti-oppressive frameworks call attention to structural injustices which make it difficult for Latinas to access resources like preventive care, health insurance, transportation, etc.

\section{Latinas and Contraception}

Some research has suggested that many Latinas use contraception inconsistently or not at all (Garcés-Palacio, Altarac, \& Scarinici, 2008; Mastinter, Feinglass \& Simon, 2013; Rivera et al., 2007; Sterling \& Sadler, 2009). Some reasons for this, as identified in the literature, include limited knowledge of contraception in general, misinformation about contraception, concerns about side effects, and inaccurate perceptions about pregnancy risk- often associated with lack of knowledge/accessible information about pregnancy in general (Carvajal, 2014). Lastly, Downing et al. (2007) found that Latinas reported greater likelihood of being discouraged from childbearing by their healthcare 
providers compared with White patients, but this doesn't necessarily imply being offered choices with regards to contraception, or that providers had meaningful conversations with Latina patients about how to avoid unwanted pregnancy.

In their study of sexual and reproductive health disparities in a sample of college students, Buhi et al. (2014) found that Hispanic students had lower odds of reporting birth control pill use or other contraceptive methods. They also suggest that disparities in contraception use are related to (1) Hispanic culture and its patriarchal system (2) traditional views of "Hispanic" women in Hispanic culture (3) acculturation (4) the desire for larger families. There is a robust body of scholarship and research specifically related to HPV and Latinas which often cites these aforementioned constructs to help explain why HPV specifically is so prevalent in Latina populations. I contend that it is urgently necessary to unpack what is meant by "Hispanic culture's" impact on health disparities as well as the other findings from Buhi et al., (2014).

In Gilliam et al.'s (2004) qualitative study of 40 Latina women ages 18-26, the majority of whom identified as Mexican American, researchers found that perceptions of side effects of contraception, combined with personal experiences with contraceptive side effects, were some reasons for not using or discontinuing use of contraception. Latinas in this study reported incorrect knowledge about contraception. Latinas communicated concern about side effects, method switching and discontinuation due to side effects, misinformation about contraception and risk of unintended pregnancy, to be barriers to contraception use. Widespread acceptance of condom use was also reported by participants (Gilliam et al., 2004). 
Similarly, in their qualitative study of 16 Latina immigrants ages 15-24, residing in Baltimore, Carvajal et al. (2017), found that the desire to prevent an unwanted pregnancy was the primary driver for contraceptive use amongst participants in their study. They also found that primary care physicians "should develop trust and foster communication by using a shared decision making approach in contraceptive counseling" for Latina women (p.158). Religious norms did not present as barriers to contraceptive use yet "positive reinforcement from family, friends, and schools" was viewed as supportive (p.158). Once again, unpacking what is meant by "religious norms" merits further analysis and exploration. Caravajal et al. (2017) used the theory of planned behavior to "guide development of the discussion guide and to frame understanding of contraceptive behavior" (p.159). This study paid particular attention to how the immigrant experience, combined with experiences of discrimination, can shape perceptions about contraception use which might differ from other groups. This is evidenced by Caravajal et al.'s (2017) findings that Latinas expressed wanting physicians to elicit and understand contraceptive preferences through a shared decision making approach instead of making ethnicity based assumptions and just prescribing and choosing a contraceptive method for them.

Sexual Health, Sexuality \& Sexual Well Being

In the past twenty years, scholarship in the area of sexual health has increasedthere are text books and peer reviewed journals dedicated to the topic, as well as robust program development to address sexual health disparities in various communities 
although this scholarship is slow moving in social work specifically (Bay-Cheng, 2013; Dodd \& Tolman, 2017; McCave, Shepard \& Winter, 2014; Sandfort \& Ehrhardt, 2004). Inevitably, the terms sexual health, sexuality and sexual well-being mean different things to different people in different contexts, as does the meaning of "sex" itself. Conceptual difficulties exist in defining the term sexual health. Sexual health as a concept will continue to evolve over time, and that definition will be contingent upon the social, political and economic contexts in which they are discussed (Edwards \& Coleman, 2004; Epstein \& Mamo, 2017; Ford et al., 2017; Giami, 2002; Sandfort \& Ehrhardt, 2004). To first be able to discuss sexual health, it makes sense to begin with the definition of health, as the definition of health in general impacts evolving definitions of the term sexual health.

The World Health Organization (WHO) plays a major role in defining health related concepts and often plays a major influencing role in defining a host of concepts related to health in general. At a conference in 1946, WHO defined the term health as "a state of complete physical, mental and social well-being and not merely the absence of disease or infirmity." Although criticized for its vagueness, this definition laid the groundwork for future definitions of other aspects of health, such as mental health, reproductive health and sexual health (Edwards \& Coleman, 2004; Hensel \& Fortenberry, 2013; Sandfort \& Ehrhardt, 2004). The Centers for Disease Control (CDC) use nearly identical language in their definition of health as well (CDC, 2014). In 1975 the World Health Organization defined the term sexual health officially for the first time, and some credit WHO for the term entering the public health consciousness (WHO, 1975). As 
previously mentioned, other organizations such as the CDC have also adopted this definition. In 1975, WHO defined sexual health as:

the integration of the somatic, emotional, intellectual and social aspects of sexual being, in ways that are positively enriching and that enhance personality, communication and love.

Sexual health was therefore identified as a positive construct worth aspiring to; an integral component of overall health. Explicit in this definition is the awareness of how many different factors contribute to good "health," and must all be in harmony with one another. These definitions make it clear that physical health is influenced by emotional, intellectual, social and sexual health. To even suggest there to be "intellectual" components to our sexual existence is worth noting.

A number of social issues have contributed to the evolution of this definition. The AIDS/HIV epidemic of the 1980's combined with increased attention on issues of violence against women had significant impacts on how sexual health was conceptualized. LGBTQ activism, feminist and women's health movements also had significant impacts on definitions and understandings of sexual health. By $2002 \mathrm{WHO}$ had made many changes to this original definition and it transformed into:

Sexual health is a state of physical, emotional, mental and social well-being related to sexuality; it is not merely the absence of disease, dysfunction or infirmity. Sexual health requires a positive and respectful approach to sexuality and sexual relationships, as well as the possibility of having pleasurable and safe sexual experiences, free of coercion, discrimination and violence. For sexual health to be attained and maintained, the sexual rights of all persons must be respected, protected and fulfilled.

Explicit in the 2002 definition is mention of pleasure and freedom from violence. It also emphasized the "sexual rights of all people," inferring that transgender and LGBT individuals, people of color, and other marginalized groups are included. There is also a 
distinction between sexual health and sexual rights (Sandfort \& Ehrhardt, 2004). I am aware of the many definitions outside of just WHO; many organizations such as Planned Parenthood have similar but slightly definitions of this concept, certainly Christian affiliated organizations also have their own interpretations.

For the purposes of this paper, I use the 2002 WHO definition. Because of the direct mention of the term sexuality to define sexual health, it then becomes important to also define sexuality, a concept that has numerous definitions. In 1987 as part of a WHO conference on sexual health, Langfeldt \& Porter (1986) defined sexuality as:

An integral part of the personality of everyone, man, woman, and child. It is the basic need and an aspect of being human that cannot be separated from other aspects of life. Sexuality is not synonymous with sexual intercourse, it is not about whether we have orgasms or not, and it is not the sum total of our erotic lives. These may be part of our sexuality but equally they may not. Sexuality is so much more; it is in the energy that motivates us to find love, contact, warmth and intimacy; it is expressed in the way we feel, move, touch and are touched; it is about being sensual as well as being sexual. Sexuality influences thoughts, feelings, actions, and interactions and thereby our mental and physical health" (Langfeldt \& Porter, 1986 as cited in Edwards \& Coleman, 2004).

Distinctions are made between sex/sexual intercourse and sexuality itself. Sexuality in this definition is a core part of everyone's being and personality, and has direct effects on all aspects of health throughout all stages of the lifespan. Sexuality influences the many ways in which we interact with our physical and social environments and with others. As Edwards \& Coleman (2004) have noted, this definition highlights an important distinction between biological aspects of sexuality and other concepts such as pleasure and stresses the importance of human connection to overall health.

The purpose and importance of having a definition of sexual health is to adequately inform programs and interventions which promote sexual well-being (Giami, 
2002). What is particularly striking about the WHO's 2002 definition of sexual health document is its emphasis on the right to sexual information and the right to pleasure as defining factors of the term sexual health. It also highlighted three important elements within the definition of sexual health: (1) the concept of sexual health varies depending on geographic location and must be understood within the context of social, political and economic climates, (2) the United States has a considerable history of social regulation of sexuality, with some groups being encouraged to procreate while others have been forcibly sterilized, (3) sexual health as a political issue has in fact preoccupied politicians and lawmakers for some time (WHO, 2016). WHO has set forth very important guidelines with regards to sexual health promotion, but these guidelines will be nearly impossible to execute with limited data about how to promote sexual health and which groups are at the greatest disadvantage.

The first point made acknowledges the social determinants of sexual health, and that politics and economic issues deeply impact one's access to sexual health. Geographic location impacts access to education about sex, sexuality and sexual health. The second point partially acknowledges a painful history where sexual control and regulation, mostly towards women and people of color, has been used to justify oppression towards many groups. In an attempt to promote sexual well-being, attention to how this history impacts certain groups is important. The third point, about sexual health being a concern for major policy players is important, and at times throughout history policy has been about social control. An example would be limiting access to family planning and abortion through policies which create barriers instead of access to these services. 
Certainly, policy has been enacted to support women and people of color, such as current policies related to Medicaid expansion and the Affordable Care Act.

\section{Sexual Satisfaction}

Numerous sexuality researchers have documented various aspects and predictors of sexual satisfaction and most studies have focused on genereal adult populations (Higgins et al., 2004). Some evidence suggests that sexual satisfaction is related to overall life satisfaction (Stephenson \& Meston, 2015). In their study of over 700 married adults, Young \& Luquis (1998) found that sexual satisfaction could not be compartmentalized to only sexual interactions (e.g. number of orgasms, frequency of sexual activity) but was also correlated with non-sexual relationship aspects such as love, trust, and communication. Similarly, Litzinger \& Gordon (2005) found a significant interaction between communication and sexual satisfaction in their study of 387 married couples. Litzinger \& Gordon (2005) found that if couples are successful at verbal communication, sexual satisfaction failed to contribute to marital satisfaction. However, they also found that even if couples were unsuccessful with communication but are sexually satisfied, they will still report high levels of martial satisfaction. Young et al. (1998) found that married women who report high levels of sexual satisfaction also reported higher levels of relationship satisfaction. In their nationally representative sample of 2250 Finnish adults, Haavio-Mannila \& Kontula (1997) found several correlates to sexual satisfaction such as young age, nonreligious upbringing, early sexual 
debut, high education, reciprocal feelings of love, and frequent intercourse. None of these studies explored gender and race/ethnicity differences with regards to sexual satisfaction.

Haavio-Mannila \& Kontula (1997) briefly explored gender differences and found that women in their sample on average reported lower levels of sexual satisfaction than men, which the authors attributed to a later sexual debut, conservative sexual attitudes, and lack of sexual assertiveness. In their study of 2168 university students in the US, Higgins et al. (2011) found significant correlates of both physiological (sexual) and psychological satisfaction which included sexual guilt, sexual self-comfort, self-esteem, relationship status and sexual frequency. Generally speaking, the direction of association was positive, except with regards to sexual and psychological satisfaction and sexual guilt, where the association was negative; higher levels of sexual and psychological satisfaction were associated with lower levels of sexual guilt. The Higgins et al. (2011) study found significant gender differences between men and women, with women reporting fewer orgasms during sexual intercourse and more conservative attitudes about sex and sexuality.

Factors consistently associated with sexual satisfaction include age (younger populations report higher levels of sexual satisfaction) (Carpenter \& Nathanson, 2009), frequency of sexual activity and orgasm (Haavio-Mannila \& Kontula, 1997), relationship status (Young et al., 1998), more permissive sexual attitudes (Haavio-Mannila \& Kontula, 1997), psychological wellbeing and fewer depressive symptoms (Dundon \& Rellini, 2010; Henderson, Lehavot \& Simoni, 2009) and sexual function or dysfunction (Dundon \& Rellini, 2010). 
A growing body of evidence suggests that positive sexual experiences are associated with other sexual health markers. For example, in their qualitative study of 20 women ages 16-18 from southern England, Hogarth \& Ingham (2009) found that young women who masturbate are more likely to report consistent contraceptive use and positive communication with their partners in contrast with women who have never masturbated. In their nationally representative sample of 3,800, ages $18-60$, and predominantly White sample, Herbenick et al. (2009) found that women who reported using vibrators currently or in the past were significantly more likely to have had a gynecological exam within the past year compared to women who had never used vibrators. Latina representation in all of these studies is very small, if at all. However, these findings clearly have implications for future work.

The term "sexual subjectivity" is often present in studies on sexual health and has been described as a "person's experience of herself as a sexual being, who feels entitled to sexual pleasure and sexual safety, who makes active sexual choices and who has an identity as a sexual being" (Toleman, 2002, as cited in Mann, 2016). In their analysis of Add Health Wave I data, Cheng et al. (2014) found that expectations of pleasure and sexual self-efficacy predicted young adult well-being in three distinct domains. Cheng et al., (2014) found that youth with higher levels of reported self-efficacy were more likely to consistently use condoms during heterosexual sexual activity and more likely to avoid unwanted pregnancy; they also found many benefits of sexual subjectivity and identified it as a resource not easily available to everyone. They propose policy driven efforts to expand comprehensive sexual education with a focus on pleasure and desire and sexual subjectivity for young women. Although this study did not focus on Latina youth 
specifically, it has several implications and areas to build upon in future work. Specifically, as I look at preferred birth control options for this sample, Cheng et al.'s (2014) study indicates that such behavior is intertwined with sexual satisfaction.

Unpacking Acculturation, Machísmo y Marianísmo

The high rates of STIs and other undesirable consequences of sexual activity can be attributed to systemic injustices, however, as previously mentioned in this literature review, Latina "culture" reappears in many studies as a factor maintaining certain health disparities. The concern is that culture becomes pathologized in the same way that stereotypes about Latinas and sex are perpetuated in the media. Latinas, and Latinos as well, are often portrayed as hypersexual (Gónzalez-López \& Vidal-Ortiz, 2008) or traditional and subservient. It's not that portraying Latinas as sexual beings is in itself problematic, it's that hypersexuality is often "conflated with non-white female bodies" (Schaab, 2017, p.574). The function of cultural stereotypes about women of color, especially darker (than white) women, is that it reduces "an individual's complex identity to a sexualized physical entity" effectively placing women of color as "objects to be claimed, conquered and consumed as desired" (Schaab, 2017, p.574). Often times current understandings of machismo and Marianísmo in particular, reify these cultural stereotypes of the oversexualized brown body. Additionally, the hypersexualization of the Latina body usually occurs in the context of youth, and Schaab (2017) argues that the result is scholarship which overwhelming focuses on younger populations. As the majority of literature on Latina sexuality also focuses on younger populations, sexual health concerns of adult and older adult populations remains understudied. This in turn 
reifies another sexual binary: the hypersexuality of young women of color vs. asexual aging women of color (Schaab, 2017). In this section, I will focus specifically on three constructs present in the body of social science literature on sexual health and Latinas: acculturation, machísmo and marianísmo.

Acculturation

Acculturation has been used to help explain health disparities related to Latina/o health issues. Acculturation is often understood as the process by which immigrants adopt a series of beliefs, attitudes, and practices as well as the language of a new dominant (Afable-Munsuz \& Brindis, 2006; Finch, Do, Frank, \& Seeman, 2009; PerezEscamilla, 2011). A great deal of literature on acculturation assumes Latinas who do not assimilate to American ideals and behaviors suffer from poorer health outcomes (AfableMunsuz \& Brindis, 2006). This is almost always attributed to Latinas who are considered foreign born. Conversely, some research and scholarship suggests that Latinas who do not assimilate have better health outcomes than their less acculturated peers. The handling of this term with respect to Latinas and their sexual and reproductive health is often confusing.

In many studies, English language proficiency and/or English language preference are proxy's for acculturation (Baezconde-Garbanati et al., 2013; Kepka et al., 2010), since measuring the construct of acculturation is challenging and not yet well defined. Acculturation scales exist, but also often utilize some measurement of English language proficiency/preference as a variable within a scale (Alvarez \& Villarruel, 2015; Castañeda, 2017). Some research trends dichotomize acculturation: Latinas are either 
acculturated and/or modern versus unacculturated, and/or "traditional" (Juarez \& Kerl, 2003). There is no continuum of this measure. Although this project is not assessing the direct impact of acculturation on sexual satisfaction or sexual health; I will explore how citizenship status, language and country of origin are correlated to sexual health and sexual satisfaction.

The over-reliance on English language proficiency to capture acculturation is interesting, and perhaps misleading. Through processes like colonialism and the system of capitalism, many of us of Latin descent lose our abilities to speak our native language through processes of assimilation and cultural appropriation and the pressure to be "more American," a reflection consistent with the central tenets of CRT. A true irony exists in the US where white students are encouraged to speak Spanish and Latina/o students are encouraged not to. In many situations, Anglo-identified individuals speak Spanish better than we do! In an increasingly xenophobic United States, where the dominant culture often receives messages about fearing a Latina/o invasion, a concept promulgated by the current US president, perhaps holding onto one's culture is not about acculturation and assimilation to mainstream US norms and values, but rather a strategy of cultural preservation and resistance against the effects of historical trauma and imperialism. What has been far less researched is the impact of enculturation: "individual processes or changes resulting from (re)socialization to indigenous cultural norms" (Alamilla et al., 2010, p.58). It is possible that there are also strong connections and associations between enculturation and sexual health which have rarely been explored.

It is also worthwhile to mention the phenomenon of the "immigrant paradox," which suggests that Latina/o immigrants coming to the US actually experience better 
health, despite all the barriers to healthcare (Guarini et al., 2011; Teruya \& BazarganHejazi, 2013). After about 7 years, their health outcomes begin to mirror that of those born in the US. The reasoning is that immigrants have protective factors like stronger social and cultural ties and behaviors which keep them healthy. The longer they are in the US, the social and community ties dissipates due to living far from relatives, working multiple low wage jobs, eating American food, and facing discrimination. It is an interesting concept in that it goes against the idea that acculturation and assimilation are good for your health. However, most studies on this concept appear inconclusive (Guarini et al, 2011): "paradox protection appears uneven and is not generalizable across races, ethnicities, age groups or genders” (Teruya \& Bazargan-Hejazi, 2013).

Machísmo

There is no one consistent definition of machísmo (Neff, 2001). Machismo is often understood as a key component within a system of patriarchy; where it encompasses certain stereotypical masculine gender roles and characteristics specific to Latina/o culture(Torres et al., 2002) and "has become a global shorthand for hypermasculinity" (Cowan, 2017,p.606). There are numerous definitions of this term which are often manipulated contingent upon the specific research agenda of those carrying out the research. It is applied liberally in the HPV literature on Latinas; it is typically used to support evidence that machísmo bears with it specific negative heath consequences for Latinas/os. This construct is almost always framed negatively, and often considered an unfortunate moral failing of Latin people and "stereotypically characterized among Latinos as a universal phenomenon," (Torres, Soldberg, \& 
Carlstrom, 2002, p.166). Some understand it as an exaggerated type of sexism, particular to Latina/o culture. Machísmo is often pathologized in research paradigms, especially when the research is conducted by "culturally insensitive and linguistically limited outsiders" (Torres, Soldberg, \& Carlstrom, 2002). Rarely are mainstream definitions of this construct challenged or interrogated albeit it is so often linked with negative health outcomes for Latinas/os. Contemporary feminist scholars have attempted to draw attention to the negative and positive aspects of machísmo (Hancock, 2016; Hurtado \& Sinha, 2016; Cowan, 2017).

In their study of sexual health knowledge of Latina/o immigrants, Seal et al. (2012) describe machísmo as a type of "exaggerated masculinity." This definition hardly explains the construct and carries with it a negative connotation. Such descriptions are not uncommon, and yet the construct in and of itself so often leads to "inconsistent, contradictory, superficial generalizations and negative stereotypical characteristics as ambiguous and misunderstood as any other aspect of the Latino culture" (Torres, Solberg \& Carlstrom, 2002, p.163).

I argue that this term is largely overused and under explained in the current literature with little to no context that patriarchal oppression exists in mainstream US culture as well: "machísmo is not a phenomenon unique to the Latino culture; concepts of manhood associated with machismo are found in most cultures of the world and are nearly, but not totally, universal" (Torres, Solberg \& Carlstrom, 2002, p. 164). 
Machísmo, because of its many definitions and interpretations, is challenging to measure; which becomes a methodological issue for many researchers. ${ }^{1}$

What makes machísmo a different kind of patriarchy and/or sexism? How are the effects of sexism perpetuated by machísmo any different than other types of sexism? This is not often explored in the literature yet many scholars continue to blame machísmo for high rates of HPV and health illiteracy when talking about reproductive health issues related to Latinas. This overly generalized and highly stereotyped "concept" of Latina culture and its relation to sexuality could also be understood as a devaluing of culture; as oversimplified and Anglo-centric understandings of Latina sexuality that may translate into biased approaches to working with Latinas (Juarez \& Kerl, 2003). It also has implications for Latina women themselves, and opens the door to the internalization of oppression.

An example of challenging how machísmo is pathologized is Garcia's (2012) study of sexual communication of Latinas with their partners. Garcia (2012) found that many people presuppose that, "Latina girls are assumed to be ineffective" at sexual communications with male partners due to "Latin culture" and "machismo" (p.122). However, Garcia's (2012) findings revealed that in fact the onus of communication during negotiation of safe sex was often placed on Latinas instead of their male partners; these women often took the lead in setting not only sexual limits, but in effectively communicating and negotiating condom use and birth control, rendering them effective

\footnotetext{
${ }^{1}$ A machísmo and caballerísmo scale exist although they have many limitations. Researchers agree that any measurement of machismo must include both positive and negative dimensions of the construct.
} 
communicators and negotiators for safe sex despite their connection to "Latin culture" and "machísmo." Latina participants in Garcia’s (2012) study are neither ineffective at sexual communication nor submissive to their male partners. These women possess skills, experience, and competency to engage in meaningful and safe sexual encounters. In this example, Latinas are not victims, and Latinos are not perpetrators if they are listening and responding to their female partners needs and desires during sex.

When we assume that "Latin culture" is synonymous with patriarchy and misogyny, it perpetuates the idea that Latin culture is "backwards", something negative, something repressive and oppressive which stands in the way of sexual agency and autonomy. Cowan (2017) is addressing the historical context in which the term manifested itself less than a century ago, by Anglophone scholars and posits that the term "sustains a web of pathologizing racisms" (p.619). When providers ascribe and researchers succumb to the essentialist notions of gender, sexuality and culture, interventions can come across as patronizing, even racist, and end up having little impact on positive health outcomes.

Often Latina culture is expressed as being sexually repressive towards heterosexual Latina women without any reflection that US culture in general is often oppressive towards all women in most social and cultural venues. Sexual repression is not something unique to Latino/a culture. This is also in direct conflict with media interpretations of Latinas as hyper sexualized and sexually liberated (Asencio, 2010; Juarez \& Kerl, 2003). There is also little analysis related to Latina women who do not identify as heterosexual when the focus is solely on machismo; arguably, Latina women identify with a wide range of sexual identities and not all feel that they are on the defense 
against Latino men (Garcia, 2012). This is interesting because obviously Latina women do not only partner with Latino men. A more nuanced discussion should instead focus on structural patriarchy implicit within social, political and economic systems which adversely affect women's health.

Some scholars offer alternative interpretations and applications of machísmo. Torres, Solberg \& Carlstrom (2002), Hurtado \& Sinha (2016) and Cowan (2017) offer a more complex understanding of machísmo and reject dichotomous interpretations of this construct. Hurtado \& Sinha (2016) "challenge the prevailing notions of Latino machismo, sexism, and homogeneity" by presenting narratives of Latino men who do not embody stereotypical notions of Latino hyper masculinity (p.1). Hurtado \& Sinha (2016) intentionally chose to present stories of educated Latino men to demonstrate the "possibility of feminist masculinities" emerging amongst younger generation of Latino men (p.2).

Torres, Solberg \& Carlstrom (2002) argue that that it is important to recognize the positive aspects associated with machismo such as strong sentiments of responsibility, protectiveness, and integrity. We are urged to consider the dynamic and multidimensional elements of machismo and that "behavioral manifestations are neither essentially positive or negative" but rather fall on a continuum (Torres, Solberg \& Carlstrom, 2002, p. 166). They suggest that interpretations of machísmo consider that "conventional conceptualizations and measures of gender role identity may have limited applicability for the Latino population as a whole" and that researchers and practitioners must be aware of the positive and negative qualities associated with this term (Torres, Solberg \& Carlstrom, 2002, p.175). Cowan (2017) takes this a step further by acknowledging the 
racist underpinning of the word, and questions how this construct is measured and applied in the social sciences and connects widespread acceptance of the term to "twentieth century anxieties" about immigration and overpopulation (p.606), an approach which helps to contextualize this word and its meaning, and how it is applied and used by scholars.

Marianísmo

Marianísmo, much like machísmo, is also frequently referred to as a negative trait, one that renders Latinas as passive actors in their sexual experiences. Seal et al. (2012) define marianísmo as "veneration of feminine purity and strength" (p.673). Torres, Solberg \& Carlstrom (2002) identify tenets of marianísmo to suggest that women are "modest, submissive, self-sacrificing, and morally and spiritually superior" to men and thus better suited to endure suffering (p.174). Despite that a few of these traits could be considered positive qualities, marianísmo is usually framed as a negative attribute, where "traditional" women are considered passive and not as liberal as their White counterparts in the US. Gónzalez-Lopez \& Vidal-Ortiz (2008) ask if "we can continue to pursue the cultural oppositional framework (i.e. White-Anglo as Protestant vs. Latin American as Catholic) that sustains Latino as based on 'traditional' notions of family?" (p.317). I would answer no, that these two frameworks limit our understanding of the nuances, complexities and multiple identities impact the Latina experience.

Marianísmo, as an element of Latina culture, is often blamed then for Latinas taking poor ownership of their sexual health. For instance, a common example is that Latina women, in comparison to their white counterparts, are less likely to negotiate 
condom use when having sexual relations with men, suggesting that they compromise their sexual health in an effort to maintain harmony in relationships. This interpretation perpetuates the stereotype of the submissive and powerless Latina.

The discourse around the "traditional" Latina is common and prevalent in the research around Latinas and sexual health, and prominent in the literature on STIs. Conclusions are then drawn between marianísmo and a negative effect on health. One example from Moreno's (2007) study suggest that because of marianísmo, HIV positive Latinas are unable to communicate their status with their partners. However, is this not a problem for many populations and individuals impacted by HIV? How can we make these conclusions between Latina culture and silence?

A number of scholars suggest that Latina cultural norms "related to sexuality, gender and disease may be associated with this population's increased risk of STIs and cervical cancer" (Fernandez et al., 2009, p.608). For example, Fernandez et al. (2009) suggest that Latina women are "traditional" and in that they value monogamous relationships with men and are therefore submissive to their male partners. Similarly, Deardorff et al. (2010) found that the importance of female virginity as conceptualized through marianísmo was linked to older Latina youth's nonuse of condoms; the assumption here is that something particular to Latina culture makes Latinas "value" virginity more than other groups. However, considering many abstinence only sexual education programming in the US, it is clear that "virginity" isn't only valued by the Latinx community. These findings are similar to Fernandez et al.'s (2009) that found in their study that married, heterosexual women reported low use of condoms in their sexual relationships with their husbands, as "trust" was a value particular to them because of 
their culture of origin. I would argue that in most cultures, especially US culture, heterosexual married women, even white women, seldom use condoms for the same reason. I do not think this is something specific to Latina cultures. I bring this to light because assumptions and stereotypes are made of Latina cultures that are often present in most other cultures as well. Would we call white heterosexual married women who don't negotiate condom use with their husbands "traditional"? Highlighting something like this as inherently "Latina" is problematic and actually untrue, and thereby urges interventions to focus on incorrect aspects supposedly "unique" to Latinas when in fact they are not. It also ignores other aspects of culture and systemic oppression against non-whites, which obviously contribute to poor health amongst people of color. What also becomes common in research articles are blanket statements such as, "given Latinas' relatively low knowledge of HPV and cultural norms inhibiting condom usage ... (p.753)" without any exploration or explanation of what the authors mean by "cultural norms" (Reimer et al., 2013). Casual references to some aspect of Latina culture permeate the literature and serve to reify white heteropatriarchy within US understandings of the relationship between health and ethnicity.

Juarez \& Kerl (2003) have suggested that although there are scholars challenging the powerlessness that accompanies the term marianísmo, most research studies rarely do so. Sex for the Latina within these frameworks becomes a duty, something which she herself cannot enjoy or embrace. We then re-enter a space of dichotomies, where Latinas are either traditional, unacculturated, and uncomfortable with sex versus liberated, acculturated, and active players in their sexual lives. However, Latinas are rarely perceived in the space occupying the latter, and if Latinas are perceived as sexually 
autonomous, then they are relegated to being considered sexually promiscuous in a sociocultural climate where sex and sexuality discourses are still framed as negative, where sex is dangerous (Bay-Cheng, 2010).

Additionally, most of the literature reviewed for this paper demonstrated underlying assumptions of heterosexuality. At the very least, participants are often assumed to be heterosexual. Although the Add Health data set does ask a question about sexual orientation, it does not ask about gender identity, and representation in this sample of Latinas who do not identify as heterosexual remains low. The lack of probing around these topics reify hetero patriarchal narratives. Even in the research realm, seldom have scientists confronted heteronormative narratives which in and of themselves serve to further marginalize Latinas who do not identify as heterosexual. Bay-Cheng (2010) argues that when women of color "lack resources, heterosexual relations may not only be a means to embodied pleasure or interpersonal intimacy, but may also serve the pragmatic purpose" of meeting one's basic needs (p.99)." Bay-Cheng (2010) goes on to encourage us to consider that heterosexuality for women of color is also a means of transitioning successfully into adulthood, especially when achieving financial independence and education feel so out of reach for this population.

When we unquestioningly accept negative attributes of marianísmo, we continue to perpetuate a heteronormative assumption about Latina sexuality deeply rooted in patriarchy. Additionally, we begin to further ascribe to a myth that only heterosexual Latinas require education about STIs, thus most interventions are not tailored to or inclusive of women who engage in sexual practices outside of heterosexual sex. An example of this is the lack of content about sexual minorities in sex education classes for 
Latina youth (Asencio, 2010; Bay-Cheng, 2010; Garcia, 2012). Future work must consider specific sexual health needs for LGBTQ identified Latinas.

\section{Conclusion}

In this chapter, I have provided a review of the literature on sexual health disparities in Latina populations, including relevant statistics about STIs and contraception use. I have defined the terms sexual health per the World Health Organization's definition, and provided an overview of the literature on sexual satisfaction. Again, few studies have studied this construct in Latina specific populations. I have unpacked and questioned cultural constructs of acculturation, machísmo and marianísmo present in the sexual health literature on Latinas. 


\section{CHAPTER 3: THEORY}

The following section explains my conceptual and theoretical framework which will guide analysis and interpretation of this proposed study. Conceptual and theoretical positioning is key to understanding the focus of these research questions and the frameworks serving as the foundation for the interpretation of findings. This is especially important for this project as it is a secondary data analysis and I, therefore, had no role in the original study design. Research begins with paradigms and perceptions about what the researcher thinks they know about the world and what they hope to learn. To this end, I will describe social constructionism and reproductive justice as conceptual frameworks informing my epistemological approach to data analysis and interpretation. Intersectionality and Latina Critical Race Theory (LatCrit) guide and center my analysis in a social justice focused research agenda.

I am fully aware of the many limitations involved in identifying a quantitative study as "intersectional." Scholars have identified the many pitfalls of calling a quantitative study "intersectional," and that even though some claim to be so, they often fail to use truly intersectional approaches, demonstrating the challenge of such an application of theory to method (Bowleg, 2008; Reisen et al., 2013). Additionally, this being a secondary data analysis, it is unlikely that it was designed with intersectionality in mind. I do not resolve to make the claim that this proposed study will be purely intersectional; however, I am committed to integrating these conceptualizations into multiple parts of this study as much as I am able. At its core, an intersectional study must "attend to the power and inequality tied to the experience of multiple social categories" (Else-Quest \& Hyde, 2016, p.321). I am starting with this premise, that I will be attentive 
to issues of power and inequality at every stage of theory application: from the literature review, through variable and statistical test selection, onto interpretation of findings.

Scholars have made a case for rejecting the practice of pulling from one single theoretical approach and instead have called for a critical pluralism of various theoretical perspectives (Hill-Collins, 1990; Mehrotra, 2010). The following is exactly that, a thoughtful confluence of different but similar conceptual and theoretical perspectives informing my epistemology and plan for data analysis. Although distinct, social constructionism, reproductive justice, intersectionality, and Latina Critical Theory all have overlapping components which complement each other well when combined. I have intentionally made distinctions between conceptual frameworks influencing my epistemological approach to the topic of Latina sexual health and theoretical frameworks. I understand conceptual frameworks as approaches influencing my paradigmatic approach to the project as the researcher. The theoretical frameworks are theories which have been tested and applied by previous researchers and guide the analysis and interpretation of data.

\section{Theoretical Frameworks}

\section{Social Constructionism}

Loosely defined, social constructionism refers to "any social influence on an individual experience" (DeLanater \& Hyde, 1998, p. 13). In short, reality is socially constructed. DeLanater \& Hyde (1998) define the constructionist paradigm by the following 5 components: (1) our experiences of the world are ordered, and we experience it as an objective reality (2) we make sense of our world through language, (3) the reality 
of life is shared among us, (4) shared experiences of reality become institutionalized and (5) knowledge can be institutionalized at the societal level or among subgroups. I plan on applying this paradigmatic framework through which to discuss Latina sexualities, as I acknowledge the social construction of gender and race. Although gender construction is not a focal point of this dissertation proposal, it is challenging or rather, nearly impossible, to discuss sexuality without also acknowledging gender as socially constructed. Social constructionism is a framework that challenges assumptions that gender and sexuality are naturally occurring categories independent from one another, but are interconnected concepts. Instead, social constructionism reframes this by suggesting that sexuality and gender are socially constructed categories, which privilege some groups over others. Sexuality is then given shape and meaning by larger social, political and historical forces marked by struggles for power (Garcia, 2012) and acknowledged through a shared experience. In the application of social constructionism to sexuality, sexuality constitutes modes of inequality which structure experiences and opportunities (Garcia, 2012). But on that same note, sexuality can also be a vehicle for empowerment and transformation. Gender certainly influences sexuality and sexual expression. Latina sexuality is also a socially constructed phenomenon where harmful stereotypes are often perpetuated through policy and popular culture. Latina sexuality and in turn reproduction is often defined by others as problematic and a site for regulation. Latinas are often considered "excessively" fertile, stereotypes perpetuated by depictions of them as wanting large families and refusing to use birth control (Garcia, 2012; Vargas, 2010). This has been used to justify forced sterilizations of Latinas as well unethical testing of birth control in this population (Garcia, 2012; Mitchell, 2010). An acknowledgement and 
recognition that gender and sexuality are socially constructed concepts, holds the potential for empowerment and transformative positive change, but can often place some groups at a social disadvantage.

Reproductive Justice

Reproductive justice as a theoretical framework informs my epistemological approach to analysis and interpretation for this project and is well suited to help conceptualize my research questions. Because reproductive justice comes from a theoretical framework which aims to understand "the complete physical, mental, spiritual, political, social, environmental and economic well-being of women and girls" (Gurr, 2011; Higgins, 2014), it is well suited to help better understand overlooked dimensions of sexual health for women of color. Reproductive justice combines a theoretical paradigm with an activist model to work towards eliminating oppression of women and women of color in various social, familial and economic domains. Reproductive justice, much like Latina Critical Race Theory, builds on the acknowledgement that many communities, in particular immigrant communities and communities of color, have experienced reproductive abuses (Higgins, 2014). Reproductive justice begins and builds from a historical viewpoint that first acknowledges that women of color have experienced reproductive abuses (such as the breeding of slaves, forced sterilizations, cash incentives/welfare benefits in exchange for long acting contraception). Reproductive justice begins with a premise that the main reproductive challenge facing women of color "are socio-economic and cultural inequities which provide some people with easier access to self-determination and bodily 
autonomy than others" (Higgins, 2014, p.5). As such, connections between reproductive justice and health and well-being are clear and strong.

Reproductive justice as a framework and lens through which to understand various social problems and as an influencer of praxis emerged as a counter dialogue to the reproductive rights movement and second wave feminisms, platforms primarily dominated by White, affluent women (Gurr, 2011). The reproductive movement of the late 1960's and early 1970's primarily focused on abortion and contraception, ignoring many of the needs of women and communities of color, in particular, immigrant women (Smith, 2005). It does make sense to put abortion access at the forefront of debates around sexuality and agency, but the reproductive justice movement emerged in response to a perceived lack of attention to unique needs of women of color and functioned to challenge various forms of structural inequalities by calling attention to other reproductive justice issues beyond only abortion.

Reproductive justice as a framework includes thoughtful considerations of all forms of oppression, and is broad in that it includes a variety of issues faced by individuals who identify as female and/or who have a uterus. The emphasis on education, in particular, sexual education for men and women, is a central tenet of the action focused element of reproductive justice (Alzate, 2009) ${ }^{2}$. Reproductive justice frameworks inform the understanding of links between education and healthcare access and poorer health outcomes. Reproductive justice also emphasizes spiritual, emotional and mental wellbeing as central to good health. No doubt sensuality, sexuality, pleasure and desire are

\footnotetext{
${ }^{2}$ In this context, I am referring to comprehensive sexual education programming.
} 
equally important components to sexual health and well-being. Considering Latinas have poorer educational outcomes compared to other groups, inclusive of access to sex education, a reproductive justice lens can aid in understanding systems of oppression which lead to poor health outcomes (Castro-Salazar \& Bagley, 2012; Higgins, 2014). Historically, women of color have been demonized for their sexuality. This continues today. The dominant culture, through popular media especially, contributes to the eroticism and exploitation of the brown and black body. Harmful health and social policies continue to restrict women of color from being sexually healthy and almost always ignore dialogue about pleasure and desire. In this instance, I am referring to policies which restrict access to contraception, health insurance and abortion. Reproductive justice calls attention to the many ways in which women of color are deemed immoral for expressing and embracing sex and sexuality. Latinas as well as other women of color are often overly sexualized, reduced to body parts instead of full complicated people with rights to pursue pleasure and sexual satisfaction (Faulkner, 2003; Hernandez, 2009).

An example of reproductive justice work in action are the group, California Latinas for Reproductive Justice (CLRJ, 2018). This coalition of people are dedicated to community education, policy advocacy and community informed research (CLRJ, 2018). They specifically conduct programming to advocate for health care, culturally relevant services, promoting comprehensive education, increasing the Latina health professional pipeline, ensuring access and equity to contraception and especially in promoting culturally unbiased research on Latinas and reproductive health (CLRJ, 2018). This organization is one example of how reproductive justice issues and values are 
transformed into praxis. Their specific focus on the need for culturally unbiased research and to increase the Latina health professional pipeline is extremely relevant to this overall project, and substantiates the contributions these findings may have for Latina reproductive health. This organization is only one example of the important work reproductive justice informed organizations are doing to advance the sexual and reproductive health needs of women of color.

Intersectionality

Intersectionality and the concept of "inter locking oppressions" is sometimes considered a theoretical approach, theory, lens, framework, method, perspective or paradigm (Buikema, Griffin, \& Lykke, 2011; Hulko, 2009). In other scholarly circles, it is accepted as a theory (Else-Quest \& Hyde, 2016). The concept of intersectionality is the lens through which I will be analyzing and interpreting this Add Health data. Although certainly theorists, writers and scholars have alluded to the concept of inter locking oppression in their work, Kimberle Crenshaw, a critical race theorist, is credited with coining the term intersectionality during the late 1970's and through the 1980's (Buikema, Griffin, \& Lykke, 2011; Cole, 2009). Although intersectionality’s history cannot be organized into a specific time and place, it is very much a product of social movements and feminist activist work which began in the 1960's and continues on through the present, and emerged as a counter-narrative to white, second wave feminisms (Collins \& Bilge, 20112); the idea of analyzing race, gender and class identities together has existed for some time (Hancock, 2007). It is worth noting that many feminist scholars of color had been using the language of inter-locking oppressions prior to 
Crenshaw's work. It began as an academic discourse which emerged out of anti-racist feminist dialogue and scholarship (Buikema, Griffin, \& Lykke, 2011; Collins \& Bilge, 2012; Erel et al., 2011; Hancock, 2016). Intersectionality identifies that the intersection of people's multiple identities leads to the justification and perpetuation of racism, sexism, classism, homophobia, transphobia in various systems through which people interact. The confluence of one's various identities can be understood through micro and macro manifestations. Racism, discrimination, and oppression can be present itself in everyday interpersonal interactions, commonly understood as micro aggressions. Structurally we can see how racist and discriminatory policies can also negatively impact people who occupy various social locations through an understanding of lack of access to healthcare and overrepresentation of people of color in carceral settings. Intersectionality instigates a re-thinking of the binary between oppressor and oppressed which "obligates us to reconceptualize power more broadly" (Hancock, 2016, p. 89).

Latina and Chicana feminists have made significant contributions to the development of intersectionality (Enns, 2010; Garcia, 1989; Higgins, 2016; Hurtado, 2001). Consistent with LatCrit theorizing, Chicana feminisms "underscore the consequences of colonialization, religion, language, land theft, and immigrant statuses related to the intermingling of Mexican, Spanish and US cultures" (Enns, 2010, p.334). Chicana feminisms have paid special attention to the concept of being at the cross-roads of various cultures, metaphorically and sometimes physically straddling two borders (Anzaldúa, 1987; Hurtado, 2001). These concepts align naturally with feminist understandings of inter-locking identities. Chicana feminists, much like Black and Asian 
American feminists, confront the issue of ending sexist oppression within the larger umbrella of ending racial oppression (Garcia, 1989).

The majority of intersectional research to date has used qualitative methods (Reisen et al., 2013), although this is changing. Many scholars especially in the health sciences are beginning to utilize intersectionality to help frame research questions, inform statistical methods when possible and to guide analysis and interpretation. Intersectionality has the ability to disrupt essentialist assumptions about Latinas and Latina culture in a way that is not demeaning and tone deaf by insisting we question assumptions about race and culture and think critically about the purpose they serve in supporting the status quo. In this study, I am focusing on the application of intersectionality in the formulation of my research questions, and approach to analysis and interpretation of findings.

Some of the limitations of the application of intersectionality to quantitative research methods include issues with statistical modeling and being constrained by additive approaches, such as attempting to understand the aspects of social identity which most impact experiences of oppression (Reisen, 2013). For example, in statistical analyses, the focus can be on isolating and controlling for various effects, which other researchers have suggested may remove context from our understanding of various social problems to a reality than can be "known." It also may be unhelpful in understanding the complexity of people's lived reality. However, the concept of inter-locking oppressions is very useful in identifying which variables to test and how to interpret findings. Intersectional theorizing and application to quantitative work can perpetuate a thorough investigation of complex social conditions, identities, and phenomena (Reisen, 2013). 
Hancock (2007) states that not only does intersectionality meet the requirements of a theoretical construct, but it also provides insights into how to conduct empirical research, as categories should matter equally, and the interactions of these categories should be dynamic and nuanced. Intersectionality "moves beyond the assumption that health outcomes may be caused by a number causes" by asserting that numerous factors are always simultaneously at play in health outcomes (Hankivisky \& Christoffersen, 2008, p.276).

The public health field has embraced intersectionality as a useful tool through which to better understand social inequities (Collins \& Bilge, 2016). Intersectionality's utility has much to do with its emphasis on understanding identity through a "social structural context" (Warner, 2008, p. 459). Intersectional approaches to incorporating multiple identities into research and practice have the potential to increase understanding into prevention, health and illness (Collins \& Bilge, 2016). An application of intersectionality to sexual health disparities faced by women of color helps us to understand why Latina women, of a lower socioeconomic status, might be disproportionately at risk for acquiring certain STIs, unplanned pregnancy, violence, coercion, etc. Lack of access to health care, health insurance, education, employment, reproductive health services are all factors that "intersect" which increases the burden of illness and disease on Latina populations. Similarly, intersectionality can guide research design in addressing aspects of sexuality that often go unaddressed in certain health disparities projects, such as addressing pleasure and sexual satisfaction as it manifests itself within communities of color. 
Concepts such as power, inequality and privilege are essential in understanding the language associated with intersectionality. Cole (2009) asserts that categorizations of race, gender identity, and sexual orientation do not simply define groups but also function to identify similarities and differences. They function to also tell a story about historical processes which have influenced the present. In other words, social location and social categories can only be understood through a historical context which contextualizes political, economic, and social institutions. Historical and present contexts in which we live shape our interpretations and perceptions of our own experiences and those of others. Oppression is multifaceted: constructs like race, gender, sexual orientation are so complex that they do not reside solely within the identity of the individual, but are acting components of social, economic, and political systems in which we live (Cole, 2009).

The theoretical application of intersectionality works to unravel why white women have considerable privilege over women of color in terms of access, equality, and opportunity. Hurtado posits that this inequality is based on structural hierarchies in our social systems, which continue to "value" white women over women of color (Hurtado, 1996). Hurtado (1996) suggests that these differences are related to the different relationships both white women and women of color have with white men (the dominant group). Returning to the concept that people of color often hold multiple identities at the same time, sometimes occupying a space of both oppressed and oppressor, Hurtado helps to understand how one's relationship to the "most valued" and with the most power in our society (white men) can and does affect equality amongst people of different gender identities, sexual orientation, and class. 
One tenet of intersectionality is its discourse on "difference." Whereas first and second wave feminists worked to eliminate "differences" between female and male, intersectionality suggests that the elimination of "difference" in feminist discourse is problematic (Crenshaw, 1993). Instead, intersectionality presupposes that the conflation of difference and ignoring intragroup differences or "colorblindness" further perpetuates oppression towards people of color and marginalized groups instead of working towards a more equal distribution of power (Crenshaw, 1993). I point this out because I am attempting to examine intra-group differences within the Add Health sample as an attempt to identify the heterogeneity within Latina groups while taking into considerations commonalities as well.

Critical Race Theory and Latina Critical Race Theory

Critical Race Theory (CRT) originated from legal studies and critical theory studies (Delgado \& Stefanic, 2000; Delgado \& Stefanic, 2012; Stefanic, 1997). Critical theory provides unique insights into various social structures by helping to explain the importance every human being places on health and social life while understanding how historical illnesses have subjugated and oppressed marginalized groups (Graham, 2011). Legal studies developed CRT based on the notion that racism shapes our current legal system stemming from social movements associated with the struggle for civil rights. Latina Critical Race Theory (LatCrit) is an extension of this theory as it applies specifically to Latina populations; LatCrit is a spinoff of CRT as it attempts to "expose and challenge the ubiquity of discrimination and oppression that leads to disparate outcomes for Latinos in the US" (Kiehme, 2016, p.119). 
LatCrit is "concerned with a progressive sense of coalitional Latina/Latino panethnicity and addresses issues often ignored by critical race theorists such as language, immigration, ethnicity, culture, identity, phenotype and sexuality" (Solorzano \& Bernal, 2001, p.311). Similar to the inception of CRT, LatCrit evolved out of Chicana/o movements from the 1960's and 1970's when scholars and activists began working on issues from the Latina/o perspective for Latinas/os (Stefanic, 1997). An increase in the Latina/o population combined with xenophobic and anti-immigrant sentiments paved the way for Latina and Chicana scholars to begin this new body of scholarship (Stefanic, 1997). LatCrit addresses the intersectionality of racism, classism, sexism, and other forms of oppression (Solorzano \& Bernal, 2001; Stefanic, 1997). LatCrit also has a history of having a strong gender analysis and is often understood as supplementary and complementary to CRT (Solorzano \& Bernal, 2001). For these reasons, LatCrit is an appropriate theoretical lens through which to attempt to understand dimensions of Latina sexuality as it applies to sexual health. Besides the obvious application that this project is centered on Latina women, LatCrit specifically considers how micro and macro issues may play into sexual health and sexual health behaviors.

CRT and LatCrit are useful in analysis to explain the existence of health disparities as CRT suggests race is often the guiding force of the distribution of power in our society. CRT helps to contribute to an understanding of racism as a social determinant of health, thus, how racism itself can lead to poorer health outcomes for people of color (Ford \& Airhihenbuwa, 2010). CRT goes beyond theory to actionable praxis; this action component enables the applicability of social work research to real world issues (Ford \& Airhihenbuwa, 2010). CRT and LatCrit encourage the use of constant critiques about the 
racial systems around us. Critique is praxis which has the potential to (1) disrupt root causes of inequality by expanding our vocabulary to discuss poorly understood racial concepts (2) use storytelling centered in the margins (3) directly challenge injustices through policy reform, direct action, or the legal system (Ford \& Airhihenbuwa, 2010). These praxis components work to actively challenge and resist power hierarchies which are meant to subjugate people of color. CRT not only aims to understand inequities, for this paper specifically and in sexual health, but attempts to eliminate the disparities and inequities through changing popular beliefs about race consciousness (Ford \& Airhihenbuwa, 2010).

In applying CRT and/or LatCrit to sexual health disparities, perhaps prevention efforts at reducing inequities are flawed because the dominant class is not experiencing the same burden of disease and other health outcomes at the same rate at which it affects women of color. CRT values individual agency; the individual has the most insight and knowledge into their own experience. CRT conceptualizes race as a socially constructed phenomenon where the social location of the individual or community then becomes the focus. Thus, CRT offers that simply documenting health disparities is not enough, professionals and researchers must challenge root causes of these inequities (Ford \& Airhihrnbuwa, 2010). This study takes concrete steps towards challenging some health inequities faced by Latinas through rejection of cultural stereotypes and by attempting to understand a multitude of social, behavioral and structural variables impacting sexual satisfaction. 
Overlap Between CRT and Intersectionality

CRT and intersectionality are entwined, complementary and share an intimate and unique relationship with one another (Collins \& Blige, 2016). CRT has a much stronger emphasis on race alone, whereas intersectionality broadens the scope of the theory by suggesting that peoples' multiple social locations and identities impact their experience of oppression including, beyond, despite and because of race. The fact that both of these theories have praxis components, action oriented components about challenging oppression, is one of the most distinguishing overlaps of these two theories. Since feminist researchers have called for research that is both grounded in theory and action oriented, it seems logical that this is the most prominent commonality of CRT and intersectionality (Gringeri et al., 2010). Intersectionality also has similarities with central tenets of social constructionism: "systems of oppression such as race, class, and gender co-constitute one another in fluid, complex, and context bound ways that cannot be captured by such fixed metaphorical images" (Mehrota, 2010, p.421). These theoretical and conceptual approaches work in concert with each other and are united by the element of "praxis." For these reasons, they are well suited to further social work's social justice focused agendas. 


\section{CHAPTER 4: METHODS}

Introduction

In this section, I will describe the Add Health study design and background, sample and sampling procedures, and data management and specify which variables were used in analysis. I will discuss how missing data were handled as well as how I checked for assumptions for ANOVA, bivariate correlations and regression analyses.

\section{Study Design \& Add Health Background}

This is a secondary data analysis of The National Longitudinal Study of Adolescent Health (Add Health) to address abovementioned research questions and hypotheses. Add Health emerged from a congressional mandate to fund a study related to adolescent health. This study had multiple funding streams, the majority coming from the United States Department of Human Services and the National Institutes of Health.

As described by Harris \& Udry (2015), the Add Health data set is a longitudinal study of a nationally representative sample of adolescents in grades 7-12 in the United States during the 1994-1995 school year. There are four waves to this project, the most recent being Wave IV which was collected in 2008 when participants were approximately between 24-32 years of age. A more thorough description of the sampling procedure will be described in the following section.

Waves I and III focused on forces that influence adolescent health and health behaviors from various dimensions of an individual's life (relationships, family, peers, communities, etc.). The goal was to better understand the impact of adolescent behavior 
on adult health outcomes. Wave IV examined health and behavioral trajectories from adolescence into young adulthood (Harris and Udry, 2015). By the time Wave IV data was collected, participants resided in all 50 states.

Add Health gathered longitudinal survey data on respondents' social, economic, psychological and physical health and well-being. Data regarding each respondent's family, neighborhood, school, friendships, peer groups and romantic relationships were also collected to better understand the impact of adolescent behavior on adult outcomes in health and well-being (Harris and Udry, 2015). Data were collected using a combination of face-to-face interviews, computer-assisted self-interview (CASI), audio computer-assisted self-interview (ACASI), and paper and pencil interviews (PAPI).

Approximately $92.5 \%$ of original Wave I participants were located for Wave IV data collection and approximately $80.3 \%$ participated in interviews. Wave IV contains 5,114 respondents and also included the collection of biological specimens to better understand disease pathways and health behavior related to obesity, stress and health risk behavior.

The Add Health data set is not without limitations, which will be discussed in detail later, and even the Wave IV 2008 data appears somewhat dated. That being said, this is the most recent nationally representative survey which addresses sexuality across the lifespan from a theoretical framework (Dennison and Russell, 2005 as cited in Cheng et al., 2014) which also contains considerable Latina/Hispanic variability. Although Add Health is a longitudinal study, this is a cross-sectional analysis of Wave IV data as variables about sexual and relationship satisfaction were only collected during Wave IV. 
Add Health Sampling Procedures \& Sample

Add Health utilized a school-based design. Per Harris \& Udry (2015), the sampling frame came from the Quality Education Database (QED) comprised of 26,666 US High Schools. From that frame, a stratified sample of 80 high schools (which were defined as schools with a $9^{\text {th }}-12^{\text {th }}$ grade and with over 30 students) with a "probability of selection proportional to school size" (p.5). Schools were stratified by region, urbanicity, school type (public, private, parochial), ethnic mix and size" (Harris \& Udry, 2015). Seventy-nine percent of contracted schools agreed to participate, for a total of 132 schools representing 80 different urban, suburban and rural communities. Size of schools varied from fewer than 100 students to over 3,000 students. All Wave I respondents were eligible for in-home interviews at Wave IV; $92.5 \%$ were located and $80.3 \%$ participated. Wave IV data were collected in 2008 .

\section{Data Management}

Analysis of the Add Health data set requires the use of statistical software capable of handling complex survey data. I used SPSS version 23 for analysis. Add Health provides access to public use data sets for free from the Add Health website. Recoding steps to facilitate analysis with SPSS will be detailed below when relevant. Wave IV data are available for download from its website as an SPSS file. Portland State University's IRB approved this study in December 2017 under the exempt approval review process, as a formal review process was unnecessary because this is a secondary data analysis of publicly available, de-identified data. 
Sample

Using the select cases feature in SPSS, I have included participants who answered "yes" to identifying as "Hispanic" and "yes" to identifying as "female" for a sample of 287 participants. Table 1 presents basic demographic information about this sample as well as variables of interest.

Sample Weights

Add Health's study design over sampled African American respondents whose parents had a college degree. I excluded cases with missing responses to outcome and correlational variables (Tietjen, Karmarkar \& Amialchuk, 2016). Demographic Table 1 reflects this adjustment as a total of 4 cases were determined missing by SPSS from the larger Latina group and 10 cases were missing from the dependent variable, sexual satisfaction.

Variables

In the following section I will describe the variables used in analysis and their level of measurement. I will describe dependent and independent variables.

Dependent Variable

Sexual Satisfaction. Sexual satisfaction is the primary dependent variable as measured by 1 item in the survey. One statement in the "relationship in detail," portion of the survey stated, "I am/was satisfied with our sex life." Respondents had the option to answer 1-5 on a Likert scale from strongly agree (1) to strongly disagree (5). Although this variable is ordinal, it is also continuous, and being treated as such for bivariate and 
multivariate statistics in this analysis. This approach of treating a technically ordinal variable as continuous provides more flexibility for statistical analyses compared with using it as a categorical variable (Field, 2013). Additionally, to be able to call this dependent variable continuous, a requirement is that the numerical distance between each set of subsequent categories is equal, and this variable meets this assumption (Field, 2013).

Correlates (Independent Variables)

\section{Relationship Variables}

Numbers of partners ever married. Participants were asked how many people they have every married and could answer from (0) 0 persons to (4) 4.

Time involved with current partner. This was a recoded variable. I computed time involved with current partner- days, months, years- which were initially 3 different variables into one for analysis and a mean score was generated. Values closer to 0 indicate less time involved with current partner.

Relationship type. Participants were asked to identify the type of relationship they are in and could answer from (1) engaged, (2) dating exclusively, (3) dating frequently, but not exclusively, (4) dating once in a while, (5) only having sex. Enjoy doing ordinary things together*. Participants were asked if they (1) strongly agree to (5) strongly disagree if they enjoy doing ordinary things with their partner. Satisfied handling problems together*. Participants were asked if they (1) strongly agree to (5) strongly disagree if they are satisfied handling problems together with their partner. 
Satisfaction handling finances with partner. * Participants were asked if they (1)

strongly agree to (5) strongly disagree with handling finances with their partner.

Partner listens when respondent needs to talk. * Participants were asked if they (1)

strongly agree to (5) strongly disagree that their partner listens to them when they need to talk.

Love and affection. * Participants who identified being in relationships were asked to respond to the statement, "my partner expresses/expressed love and affection to me," which was measured on a Likert scale from strongly agree (1) to strongly disagree (2) (Ordinal).

Trust partner to be faithful. * Participants were asked to respond in a Likert scale from strongly agree (1) to strongly disagree (4) to the statement, "I trust/trusted my partner to be faithful to me (Ordinal).

Love. * Participants were asked to respond to the question, "How much do you love [your partner]," with a Likert scale of a lot (1) to not at all (4) (Ordinal).

Happiness in relationship. * Participants were asked, "in general, how happy are you in your relationship," with a Likert scale of very happy (1) to not too happy (3) (Ordinal).

Commitment in relationship. * Participants were asked, "how committed are you to your relationship? "with a Likert scale from completely committed (1) to not at all committed (4) (Ordinal).

Frequency of sexual relations. Participants were asked to identify frequency of sexual relations based on week (1) month (2) year (3). Depending on how this question was answered participants were then asked to identify frequency within these determined tie frames from 0-4 times. 
*Relationship Satisfaction Variable. This was a recoded variable of aforementioned (*) relationship variables computed together which generated mean scores. Values closer to 1 represent high levels of relationship satisfaction, values closer to 5 represent low levels of relationship satisfaction.

\section{Socioeconomic Status Variables (SES)}

Hispanic/Latino. Participants were asked, "are you of Hispanic or Latino origin?", and participants could answer $0=$ no, $1=$ yes.

Latino/Hispanic Background. Participants were asked, "What is your Latino or Hispanic background ?", and could choose from Mexican/Mexican American, Chicana, Cuban, Puerto Rican, Central/South American, and Other Hispanic.

Religion. Participants were asked, "what is your present religion?" and could choose from none (1), protestant (2), catholic (3), other Christian (4), Jewish (5), Buddhist (6), Hindu (7), Muslim (8), and other (9).

How important is religion. Participants were asked, "how important (if at all) is your religious faith to you?, and could choose from (1) not important to (4) more important than anything else.

Household Income. Participants were asked to identify their household income from less than 5,000 (1) to 150,000 or more (12).

Personal Income. Participants were asked to identify personal yearly income before taxes from less than 5,000 (1) to 100,000-149,999 (11).

High School Graduation Status. Participants were asked, "what is your high school graduation status?" and could answer from finished with high school diploma (1), earned 
a GED (2), earned a certificate of attendance/completion (3) and did not receive diploma, GED or other certificate (4).

Highest level of education. Participants identified their highest level of education from $8^{\text {th }}$ grade or less (1) to completed post baccalaureate education (13).

Citizenship status. Participants were asked if they were born a US citizen, $(0)=$ no, $(1)=$ yes. Depending on how participants answered, they were then asked if they are trying to become a US citizen, $(0)=$ no, $(1)=$ yes.

Have become a US citizen. Participants were asked if they have become US citizens and could answer (0) no and (1) yes.

Trying to become a US citizen. Participants were asked if they are trying to become a US citizen and could answer (0) no or (1) yes.

Health Insurance. Participants were asked about their current health insurance status, (1) $=$ no health insurance, $(2)=$ receives insurance through work, $(3)=$ receives insurance through a union, (4) = receives health insurance through school, (5) = covered by spouse's insurance, $(6)=$ covered by parent's insurance, $(7)=$ active duty/covered through military, $(8)=$ buy private insurance, $(9)=$ Medicaid, $(10)=$ Indian Health Service and (11) don't know. Table 1 reflects participants' responses as some of these categories were unmarked.

\section{Reproductive and Sexual Health Variables/Sexual Behaviors}

Sexual orientation. Participants were asked to identify the label that best represents them by answering (1) 100\% heterosexual, (2) mostly heterosexual, (3) bisexual, (4) mostly homosexual, (5) 100\% homosexual and (6) not attracted to males or females. 
Number of partners-vaginal intercourse. Participants were asked how many times they had ever had vaginal intercourse from 1 partner (1) to 5 partners (5).

Methods of birth control in past 12 months: Participants were asked to identify if they had used the following methods in the past 12 months where $0=$ no and $1=$ yes.

Participants were able to identify more than one method of birth control in the past 12 months.

Condoms. Dichotomous, 0-1.

Birth Control Pills. Dichotomous, 0-1.

Birth Control Injection. Dichotomous, 0-1.

Emergency Contraception. Dichotomous, 0-1.

IUD. Dichotomous, 0-1.

Withdrawal. Dichotomous, 0-1.

Tubal ligation. Dichotomous, $0-1$.

No method used. Dichotomous, $0-1$.

STIs. Participants were asked if they had ever been told by a doctor or medical professional if they have ever had any of the following STIs (lifetime), these are all dichotomous variables where $0=$ no and $1=$ yes and categorical.

Chlamydia. Dichotomous, $0-1$.

Gonorrhea. Dichotomous, 0-1.

Syphilis. Dichotomous, $0-1$.

Genital Herpes. Dichotomous, 0-1.

Genital Warts. Dichotomous, 0-1.

HPV. Dichotomous, 0-1. 
Analysis

This secondary analysis is a correlational, cross-sectional study (Field, 2013). I have selected a cross-sectional analysis because only Wave IV includes variables about sexual satisfaction and relationship satisfaction and because I am interested in better understanding adult sexual health outcomes. At least two recent Add Health studies set a precedent for such an approach (Sutin, Stephan \& Terracciano, 2016; Tietjen, Karmarkar \& Amialchuk, 2016); both of which conducted cross-sectional analyses using regression models of Wave IV data. I believe multiple components of this analysis are consistent with central tenets of intersectionality theory. Not only is this study specific to Latina women, but, as previously mentioned, variables selected for statistical tests reflect intersectional positioning and identities by including individual/behavior variables in conjunction with ethnicity and SES/structural variables without the presumption that one is more "important" than another. The regression models in this analysis reflect this. Intersectionality and LatCrit guide interpretation of findings. The primary objective for the following study was to examine the association of sexual satisfaction with sexual behavior variables, relationship variables and/or SES/structural variables. The secondary objective was to determine if there are differences in sexual satisfaction between Latina ethnic subgroups.

Missing Data

I began by evaluating the frequency and nature of missing data to weigh strategies such as for dealing with missing data. I began by running frequencies of categorical 
variables and descriptives of continuous variables. With regards to sexual satisfaction, treated as a continuous dependent variable, only $3 \%$ of responses were considered missing, which could be due to participants identifying as not being sexually active and/or not being in a sexual relationship at the time of participation in the Add Health survey. The SPSS default setting recognizes any blank cell as missing data automatically (Cronk, 2016; Pallant, 2013). I chose to use the listwise deletion method which, as mentioned, is also the default option in SPSS due to the small amount of missing data despite the fact that it quickly reduces sample size and statistical power.

\title{
Assumptions
}

This section outlines preliminary analyses and assumption checking for Analysis of Variance (ANOVA), correlations and regression models.

\begin{abstract}
ANOVA Analyses
Analysis of variance (ANOVA) is used to determine the proportion of variability attributed in this case to different ethnic subgroups within the larger Latina category. It compares the means of groups of participants that vary on a single independent variable. I could have potentially used T-tests to investigate group differences, however, conducting multiple T-tests at a time increases the chance for Type I errors (Cronk, 2016). As such, ANOVA compensates for the limitations of T-tests in this capacity and identifies if significant group differences exist. The one-way ANOVA requires a single dependent variable (sexual satisfaction) and a single independent variable (ethnic subgroups). Since
\end{abstract}


each ethnic subgroup was measured independently as its own variable, I computed a new variable combining all ethnic subgroups (Mexican, Puerto Rican, Central and South American, Chicana, Cuban and Latina "other") for ANOVA analysis. Although ANOVA assumes that the dependent variable is interval or ratio, I am treating the dependent variable as an interval continuous variable, which meets the assumption for ANOVA analysis. Each participant can only provide one score for the dependent variable, and this assumption has been met.

Bivariate Correlation Analyses

The non-parametric Spearman correlation coefficient determines the strength of relationship between two variables. Although the Spearman correlation is considered weaker than a Pearson correlation coefficient, it can be used in more situations, considering the categorical and ordinal nature of the majority of variables of interest. The Spearman correlation coefficient functions on the basis of ranks of data and requires ordinal and/or interval-ratio data for both variables and does not require variables to be normally distributed. The variables used for Spearman correlations in this analysis meet this assumption.

Following the preliminary analysis of variables of interest, a bivariate correlation matrix was constructed which describes the strength and directions of relationships amongst sets of independent variables (SES variables, relationship variables, and sexual health behavior variables) with the dependent variable (sexual satisfaction). Since the ANOVA analysis of group differences regarding sexual satisfaction identified no 
significant group differences, bivariate correlations were conducted on the full sample, using the continuous recoded variable of sexual satisfaction.

Regression Analyses

Multiple linear regression allows for the association between variables. As in the ANOVA analysis, I am treating the dependent variable as an interval continuous variable. I could have re-coded sexual satisfaction as a binary variable and then run a logistic regression, but I encountered methodological issues related to what to do with the "neither agree nor disagree" category and using it as a continuous variable addresses this limitation. Additionally, multiple linear regression yields more robust results than logistic regression (Cronk, 2016; Pallant, 2013).

The dependent variable should be normally distributed and a check of linearity in SPSS for sexual satisfaction shows that this assumption has been met. Dichotomous variables are also acceptable as independent variables. Linearity, normality and equal variance are also checked at the multivariate level when running multiple regression models. I checked these by conducting histograms on relevant variables, and using the residuals plot from the SPSS regression output for variables input into the regression models.

A priori criteria, in this case, statistically significant bivariate correlations where the alpha is set at .05 will determine which variables are appropriate to enter into the regression model. Any number of variables can be entered on each step, and any number of steps can be used where each step is a separate regression model. The major advantage of this approach is that a change in $\mathrm{R}$ squared is computed, allowing for a test of whether 
a significant amount of additional variance is accounted for by the variable or variables entered on each step. This multiple regression method will use statistically significant covariates to be correlated with sexual satisfaction prior to entering variables for which correlation remains unknown (Field, 2013) with the exception SES control variables. SES control variables of income, education and religion were included in regression models to account for intersectional positioning.

\section{Conclusion}

This study investigates three different sets of independent variables for analysis with sexual satisfaction as the dependent variable. Assumption checking was conducted for all variables in order to run ANOVA, bivariate correlations and regression analyses. 


\section{CHAPTER 5: RESULTS}

Introduction

In this section I will present descriptive statistics on the whole Latina sample $(n=287)$, and then provide descriptive statistics per each Latina subgroup. I will first discuss univariate analysis (descriptive statistics) about my sample and dependent variable. I will then discuss inferential statistics including ANOVA, bivariate correlations and regression analysis.

Preliminary Analysis of Variables of Interest

A full set of univariate descriptive statistics were run for all variables of interest in this dataset. The select cases function was specified to all participants who identified as female and who answered "yes" to being Hispanic (Latina). As mentioned, frequencies were run for categorical variables and descriptives of all continuous variables of interest; all variables were also explored per Latina subgroup: (Mexican $(n=161)$, Puerto Rican $(n=39)$, Central \& South American $(n=33)$, Chicana $(n=19), \&$ Hispanic Other $(n=37))$ (see demographic table 1).

For interval or ratio continuous variables, descriptives were run to show means, standard deviations as well as skewness and kurtosis statistics. Histograms were also run for each continuous variables of interest to assess normality. I am presenting histograms for age (figure 1), household annual income (figure 2), and number of vaginal sex partners (figure 3). I am also presenting the means plot for sexual satisfaction and Latina ethnic subgroup (figure 5), and these variables were all normally distributed. The number 
of vaginal sex partners and Latina ethnic subgroup was also normally distributed, except for the Puerto Rican group, where three outliers appeared after running the explore function, the histogram presented reflects the removal of these outliers (figure 3 ). Normality was assessed using the Explore feature on SPSS, where histograms and normality plots with tests indicated the distribution of each variable of interest.

With regards to correlations and assumptions, it is important that the data be normally distributed. However, because many variables of interest are not normally distributed due to the ordinal and categorical nature of many of the independent variables, the Spearman correlation coefficient is used in place of the Pearson r.

A preliminary analysis of the data was conducted where each variable of interest was examined in its original form. Means, standard deviation and range were computed for all continuous interval ratio independent variables and frequencies were run on all categorical independent variables (as well as the dependent variable).

\section{Descriptive Statistics}

Please refer to Table 1 for a complete list of the following descriptive statistics. A total of 287 Latina women (female \& answered "yes" to being "Hispanic") are included in this sample. In terms of exploring multi-racial identities, 17 (5.9\%) identified as Latina and Black, $23(8 \%)$ identified as Latina and Native American, $6(2.1 \%)$ identified as Latina and Asian and $1(.3 \%)$ person identified as Latina, Asian and African American. The mean age of Latinas in the sample is $28.9(\mathrm{SD}=1.73$, range 36-43). Regarding sexual satisfaction, the dependent variable driving this analysis, $230(80.1 \%)$ reported agreeing 
to being sexually satisfied (agree or strongly agree); the mean score of the sexual satisfaction variable the mean score was $1.83(\mathrm{SD}=1.06$, range $1-5)$, indicating a range of agree-strongly agree. Sexual satisfaction was scored from (1) which is "strongly agree" and (5) which is "strongly disagree," the closest to 1 indicates more sexual satisfaction. The mean score of total recoded relationship satisfaction variables is 1.82 $(\mathrm{SD}=.87$, range 1-5) indicating high levels of relationship satisfaction. For relationship satisfaction, (1) indicates higher levels of relationship satisfaction and (5) indicates lower levels of relationship satisfaction.

The majority of Latinas ( $\mathrm{n}=168,58.5 \%)$ reported obtaining some college, and/or having a bachelor's degree or higher. Regarding annual household income, $30.3 \%(n=87)$ reported an annual household income between $25,000 \mathrm{~K}-49,000 \mathrm{~K}$ and $45.3 \%(\mathrm{n}=130)$ reported an annual household income of 50,000 or higher (please see pie chart/figure 4). The majority of Latinas $(\mathrm{n}=191,66.4 \%)$ reported having health insurance. Most Latinas $(\mathrm{n}=228,79.4 \%)$ identified as heterosexual and few reported their relationship status as "dating exclusively" ( $\mathrm{n}=32,11.1 \%$ ). Regarding citizenship status, $82.2 \%$ of Latinas reported being born a US citizen, 33 (11.5\%) reported having become a US citizen since birth, and $14(4.9 \%)$ reported currently trying to become US citizens. Ninety-eight (34.1\%) of Latinas reported English as the language most used with family, and 76 (25.5\%) participants said Spanish was the language primarily used with family. Condoms $(\mathrm{n}=103,35.9 \%)$ and oral contraception (the "pill") $(\mathrm{n}=79,27.5 \%)$ were the two most reported contraceptives used in the past 12 months. The most prevalent STI ever diagnosed amongst this larger Latina group was chlamydia $(n=42,14.6 \%)$, followed by HPV $(n=17,5.9 \%)$ and then genital herpes $(n=12,4.2 \%)$. 
Inferential Statistics

Bivariate Correlation Matrix

Prior to running multivariate models, a bivariate correlation matrix was constructed to examine the strength and direction of linear relationships (Cronk, 2016;

Field, 2013) between the dependent variable (sexual satisfaction) and groups of independent variables (SES variables, non-sexual relationship variables, and reproductive health variables). A number of statistically significant relationships were noted and are detailed in tables 2, 3 and 4. Bivariate matrices for sexual satisfaction and SES variables and sexual satisfaction and reproductive health variables yielded no statistically significant relationships with respect to the dependent variable, however, a number of statistically significant relationships between independent variables were present and identified on tables 2 and 3. I will not describe these relationships because they are not relevant to the dependent variable of interest in this analysis. Significant bivariate correlations will guide variable entry for regression models.

\section{Bivariate Correlations: Sexual Satisfaction \& SES Variables}

There were no significant correlations between sexual satisfaction (DV) and any SES variables (Table 2). Sexual satisfaction was not significantly correlated with age, income, education level, religion or health insurance status.

\section{Bivariate Correlations: Sexual Satisfaction and Reproductive Health Variables}

There were no statistically significant correlations between sexual satisfaction and any of the reproductive health variables, although statistically significant relationships do 
exist between independent variables on this correlation matrix (Table 3). Sexual

satisfaction was not significantly correlated with sexual orientation, contraception use in the past 12 months, or STI history.

Bivariate Correlations: Sexual Satisfaction and Relationship Variables

These set of independent variables yielded the most significant bivariate correlations (Table 4). Amongst the all Latina group ( $\mathrm{n}=287)$, sexual satisfaction was significantly, positively correlated with respondent and partner enjoying ordinary things together $\left(\mathrm{r}_{\mathrm{s}}=.34, \mathrm{p}<.01\right)$, satisfaction with handling problems $\left(\mathrm{r}_{\mathrm{s}}=.34, \mathrm{p}<.01\right)$, satisfaction with handling finances with partner $\left(\mathrm{r}_{\mathrm{s}}=.40, \mathrm{p}<.01\right)$, partner listening when respondent needs to talk $\left(\mathrm{r}_{\mathrm{s}}=.35, \mathrm{p}<.01\right)$, partner expressing love and affection to respondent $\left(\mathrm{r}_{\mathrm{s}}=.32\right.$, $\mathrm{p}<.01)$, respondent trusting partner to be faithful $\left(\mathrm{r}_{\mathrm{s}}=.20, \mathrm{p}<.01\right)$, extent of respondent's love for current partner $\left(\mathrm{r}_{\mathrm{s}}=.42, \mathrm{p}<.01\right)$, extent of respondent's happiness with current partner $\left(\mathrm{r}_{\mathrm{s}}=.39, \mathrm{p}<.01\right)$, extent to which respondents are committed to current partner $\left(\mathrm{r}_{\mathrm{s}}=.38, \mathrm{p}<.01\right)$, and frequency of sexual activity with partner $\left(\mathrm{r}_{\mathrm{s}}=.31, \mathrm{p}<.01\right)$. Sexual satisfaction was negatively correlated with time involved with current partner-months $\left(\mathrm{r}_{\mathrm{s}}=-.13, \mathrm{p}<.05\right)$. Sexual satisfaction was not significantly correlated with number of partners ever married, relationship type or extent of contraceptive use with partner.

\section{ANOVA Analyses}

The means of sexual satisfaction amongst the 6 different Latina subgroups were compared using one-way ANOVA (please refer to table 5/means plot are represented in Figure 5). No significant difference was found $(F(5,266)=.26, \mathrm{p}=.94)$. Sexual satisfaction did not differ significantly amongst Latina subgroups. Due to results from the 
aforementioned ANOVA analysis with sexual satisfaction (the dependent variable) amongst 6 different ethnic subgroups, where group differences regarding sexual satisfaction were not statistically significant, I will consider the larger all Latina group for analyses moving forward.

I computed a one-way ANOVA comparing number of vaginal sex partners ever had amongst 6 different Latina subgroups (please refer to table 6/means plot are represented in Figure 6). I conducted this ANOVA after removing 4 outliers per the Explore feature on SPSS which identified that one person had indicated 200 partners, one person indicated 90 partners and two people indicated 60 partners (stem and leaf plot is represented in figure 7). After removing stated outliers, an ANOVA was calculated comparing Latina ethnic subgroups and number of vaginal sex partners, the interaction was not significant $(\mathrm{F}(5,244)=.863, \mathrm{p}>.05)$. Number of vaginal sex partners did not differ significantly amongst Latina ethnic subgroups; Mexican Latinas reported a mean of $6.20(\mathrm{SD}=6.97)$ partners, Puerto Ricans had a mean score of $7.31(\mathrm{SD}=5.34)$, Central and South Americans had a mean score of $5.70(\mathrm{SD}=6.79)$, Cuban Latinas had a mean score of $2.67(\mathrm{SD}=1.66)$, Chicana Latinas had a mean score of $5.67(\mathrm{SD}=3.52)$ and the Latina other group had a mean score of $7.64(\mathrm{SD}=7.24)$. The Latina other group had the highest mean (7.64) compared with Cuban Latinas that had the lowest mean (2.67). The mean average of sexual partners for all women was $6.29(\mathrm{SD}=7.24)$ partners.

Regression Analysis

For one regression analyses, I combined all significant relationship variables from the bivariate correlation analysis into a single continuous variable based on the mean 
score. I used the "compute a new variable" feature on SPSS to do this. I also ran a regression analyses with each of the 9 relationship variables input separately. I used income, education and religion as SES control variables to reflect intersectional positioning.

A multiple linear regression analysis was conducted to see if time involved with current partner is related to sexual satisfaction controlling for education, income and religion (Table 7$)$. The overall model was not significant $(F(3,240)=1.88, p>.05)$. Time involved with current partner, controlling for income, education and religion, is not a significant correlate of sexual satisfaction.

A multiple regression analysis was conducted to see if relationship satisfaction is correlated with sexual satisfaction after controlling for education, income and religion (Table 8$)$. The overall model was significant $(\mathrm{F}(3,242)=12.43, \mathrm{p}<.01) ; 48 \%$ variability explained by relationship satisfaction, income, education and religion. Relationship satisfaction was a significant correlate for sexual satisfaction $(B=.64, t=12.43, p<.01)$ after controlling for education, income and religion. The more one is satisfied in their relationship, the more sexual satisfaction they experience regardless of income, education or religion.

A multiple linear regression analysis was conducted to see if the number of lifetime vaginal sex partners is related to sexual satisfaction controlling for education, income and religion (table 9). The overall model was significant $(\mathrm{F}(4,227)=3.76$, $\mathrm{p}<.01)$ and $\mathrm{r}=.24$ explaining $6 \%$ of variability explained by number of vaginal sex partners, education, income, and religion together explaining sexual satisfaction. Number of vaginal sex partners is a significant correlate for sexual satisfaction $(B=.15, t=2.30$, 
$\mathrm{p}<.05)$ after controlling for education, income, and religion. More lifetime vaginal sex partners are associated with more sexual satisfaction, regardless of income, education or religion.

A multiple linear regression analysis was conducted to see if frequency of sexual activity with partner is correlated with sexual satisfaction controlling for education, income and religion (Table 10). The overall model was significant $(\mathrm{F}(4,235)=6.23$, $\mathrm{p}<.01)$ and $\mathrm{r}=.31$ explaining $10 \%$ of variability of frequency of sexual activity, income, education, and religion together explaining sexual satisfaction. Frequency of sexual activity is a significant correlate of sexual satisfaction $(\mathrm{B}=.29, \mathrm{t}=4.14, \mathrm{p}<.01)$ after controlling for income, education and religion. More frequent sexual activity leads to more sexual satisfaction regardless of income, education or religion.

A multiple regression analysis was conducted to see if enjoying ordinary things together, satisfaction with handling problems with partner, satisfaction handling finances with partner, feeling that one's partner listens when they need to talk, partner expresses love and affection for respondent, extent of respondent's current love for partner, extent of respondent's happiness with current partner, and the extent of respondent's commitment to current partner are correlated with sexual satisfaction (table 11). The purpose of running this particular regression analyses was to determine which of these aspects are most important for sexual satisfaction when entered simultaneously together into the regression model. The overall model was significant $(\mathrm{F}(9,239)=28.79, \mathrm{p}<.01) ; \mathrm{r}$ $=.73$ explaining $53 \%$ of the variability of all the variables entered together. When entered altogether, 4 variables loose statistical significance: enjoying ordinary things together $(\mathrm{B}=-.04, \mathrm{t}=-.43, \mathrm{p}>.05)$, partner listens when respondent needs to talk $(\mathrm{B}=.08, \mathrm{t}=.90$, 
$\mathrm{p}>.05)$, extent of respondent's current love for partner $(\mathrm{B}=.09, \mathrm{t}=1.12, \mathrm{p}>.05)$ and the extent of respondent's happiness with current partner $(B=.16, t=1.82, p>.05)$. In this regression model, satisfaction handling finances with partner is most correlated with sexual satisfaction $(B=.22, t=3.50, p<.01)$ based on the low $p$ value. Satisfaction handling problems with partner $(\mathrm{B}=.17, \mathrm{t}=2.14, \mathrm{p}<.05)$, partner expressing love and affection $(\mathrm{B}=.22, \mathrm{t}=2.73, \mathrm{p}<.01)$, trusting partner to be faithful $(\mathrm{B}=-.17, \mathrm{t}=-2.43, \mathrm{p}<.05)$, and extent to which respondent is committed to current partner $(\mathrm{B}=.15, \mathrm{t}=2.04, \mathrm{p}<.05)$ all remain significantly correlated with sexual satisfaction.

Lastly, a multiple regression analysis was conducted to see if number of sexual partners, frequency of sexual activity and time involved with current partner are correlated with sexual satisfaction when entered together in a regression model. The overall model was statistically significant $(\mathrm{F}(3,240)=12.86, \mathrm{p}<.01)$. When entered together, number of vaginal sex partners $(B=.16, t=2.53, p<.01)$ and frequency of sexual activity $(\mathrm{B}=.33, \mathrm{t}=5.49, \mathrm{p}<.01)$ remain statistically correlated with sexual satisfaction. Time involved with current partner $(B=.10, t=1.58, p>.05)$ is not significantly correlated with sexual satisfaction. The more number of sex partners and the more frequent sexual relations are most correlated with increased sexual satisfaction regardless of time involved with current partner.

Conclusion

In conclusion, most women across all groups reported agreeing with being sexually satisfied in their romantic and sexual relationships. Analyses revealed no 
significant group differences with regards to sexual satisfaction or number of vaginal sex partners among Latina subgroups. Relationship satisfaction, frequency of sexual relations, and number of vaginal sex partners were significantly correlated with sexual satisfaction, controlling for income, education and religion. 


\section{CHAPTER 6: DISCUSSION}

Introduction

This study sought to investigate correlates of sexual satisfaction in a sample of Latina women and to identify if any group differences in sexual satisfaction exist amongst various Latina ethnic subgroups. This study also sought to investigate if any statistically significant relationship exists between contraceptive utilization and sexual satisfaction and STI history and sexual satisfaction. Results indicate that no statistically significant relationship exists between STI history or contraceptive utilization with sexual satisfaction for any group of Latinas in this sample. This study's main findings and most important conclusions are that Latinas of all ethnic groups are sexually satisfied and that relationship satisfaction is significantly correlated with sexual satisfaction for adult Latinas. Group differences with regards to sexual satisfaction amongst Latina subgroups were not statistically significant. In the following section I will identify main points of my findings as they relate to the literature and theoretical frameworks discussed in previous chapters. I will also discuss study limitations of this secondary data analysis. I will conclude with implications for social work research, education and practice and directions for future work.

Meaningful Relationships, Sexual Satisfaction and Challenging Cultural Constructs

As per LatCrit, Latina identities have been centered in this project, and ethnic subgroup differences were explored. The main findings of this study show that Latinas of all ethnic groups are sexually satisfied, thus Latinas in this sample are a sexually satisfied 
group. This is an important finding for several reasons; first, because these results show that Latinas are experiencing positive sexual health outcomes in contrast to established health disparities around STIs and other reproductive health outcomes (CDC, 2016; Jackson, 2013; Gándara et al., 2015). This finding is also important because it challenges stereotypical notions associated with concepts like Marianísmo, which suggest that Latinas do not engage in sex for their own pleasure (Cowan, 2017; Torres, Solberg \& Carlstrom, 2002). Latinas in this study also reported high levels of relationship satisfaction, once again contradicting notions that Latinas are powerless in relationships and have little control (Gónzalez-Lopez \& Vidal-Ortiz, 2008). My findings corroborate those of Barrientos \& Paéz (2006) and Rosen \& Bachmann (2008) which also found that non-sexual relationship aspects are strongly associated with sexual satisfaction for Latina women.

Latinas in this sample are neither hypersexual nor virginal; sex is not only for men's pleasure, as Latinas value pleasurable sexual experiences. The notion of the “virginal” Latina as perpetuated by marianísmo, suggest that Latinas' relationship with Christianity renders them incapable of experiencing sexual pleasure (Torres, Solberg \& Carlstrom, 2002). However, considering an intersectionality informed analysis, and the intersection of multiple identities, Latinas can be religious and Christian and still seek out and experience sexual pleasure as demonstrated by the large Christian/Catholic affiliation by Latinas in this sample who also reported being sexually satisfied.

For Latinas, meaningful relationships significantly impact sexual satisfaction and vice versa; Latinas value equity in romantic and sexual relationships as well as communication, mutual trust and respect. Latinas are not powerless to men contrary to 
popular beliefs about the influence of machísmo on Latina sexuality (Garcia, 2012).

Latinas value partners who are respectful. They also value sex and intimacy. Sexual health promotion for Latinas needs to shift its focus away from approaching this group as "at risk" and instead focus on fostering healthy relationships for Latinx individuals.

Findings from this study corroborate previous findings which found significant correlations between relationship satisfaction and sexual satisfaction (Barrientos \& Páez, 2006; Rosen \& Bachmann, 2008) and provide evidence of the beneficial effects of sexual satisfaction on the wellbeing and interpersonal relationships for Latinas. These findings are consistent with central tenets of reproductive justice which identify that a number of factors related to the mind, body, and spirit connection impact overall wellbeing for women of color (Gurr, 2011; Higgins, 2014). Feeling trust, love, and being able to handle problems with one's partner can make people feel like they have power in relationships by feeling like their opinions are valued and heard. LatCrit is especially attuned to issues of power in micro, mezzo, and macro systems, and feeling one has power in a relationship has the potential to influence feelings of sexual empowerment and overall agency. My findings reflect that Latinas value interpersonal romantic and sexual relationships, and support a rejection of mainstream understandings of machísmo and marianísmo that render Latinas powerless and subservient in their sexual lives. These findings also push back against dominant dichotomous representations of Latina sexuality which categorize Latinas as either repressed and submissive or modern, liberated, and hypersexual (Juarez \& Kerl, 2003). I would also like to point out that "hypersexuality" is not problematic, provided it occurs in a consensual context. It is not my intention to frame hypersexuality as inherently problematic; I merely want to point 
out that the binary of hypersexual vs. virginal is not representative of Latina sexuality. That same goes for the idea of being "virginal" as it is not my intention to suggest that anything is wrong with Latinas who value this in relation to their sexual selves; merely the portrayal that Latinas are either/or with regards to this concept are what need to be challenged.

LatCrit specifically encourages challenging cultural stereotypes (Solorzano \& Bernal, 2001; Stefanic, 1997). Latinas in this sample do not easily fit in with either group; instead descriptive and inferential analyses reveal nuanced and complex identities who appear to value and engage with their sexuality and sexual lives. Juarez \& Kerl (2003) write explicitly about the impact of the internalization of the concepts; and how, as Latinas, we often unquestionably accept and internalize these own messages about our sexuality, and accept messages that sexual pleasure and desire are not for us. Cultural narratives around the "danger" of hypersexuality are quite problematic, as they detract focus away from consensual and "safe" sex health promotion which can only be achieved through comprehensive sex education for all people. Hypersexuality is not the problem: lack of access to sex education and health care, and non-consensual sex should be the focus of health promotion and intervention. These findings show that Latinas value engaging with sex, and that different aspects of relationships impact sexual satisfaction.

The finding that satisfaction handling finances with one's partner is strongly correlated with sexual satisfaction is telling because of the known wage gap between Latinas and non-Hispanic white men; where Latinas make 54 cents to their dollar (National Partnership for Women and Families, 2018; Patten, 2016) as this challenges popular myths related to machísmo where men are the bread winners and in control of 
finances in a partnership (Juarez \& Kerl, 2003). Communication and equity about how finances are handled are highly important to Latinas, and impact sexual and relational wellbeing. If Latinas are routinely paid less than white men and women, and less than other racial groups, then dealing with finances is a particularly important part of their well-being and intersects with their experiences of sexual satisfaction.

Despite my hypothesis that socioeconomic factors such as education, income, religion and health insurance status are correlated with sexual satisfaction, this study did not find this to be the case. These findings corroborate Henderson, Lehavot \& Simoni's (2009) study which found that in a sample of predominantly white, heterosexual women, SES was not associated with sexual satisfaction. They also found that relationship satisfaction is significantly impacted by social support, depression and sexual functioning, and was most closely associated with sexual satisfaction. Like Henderson, Lehavot \& Simoni's (2009) findings, the association of relationship variables with sexual satisfaction remained strong despite the addition of other variables.

Perhaps sexual satisfaction transcends issues related to class, education, and income and regardless of SES, Latina women still engage with and experience sexual pleasure and desire. Understanding SES factors and their relationship to sexual satisfaction is still necessary to discover structural factors which create conditions in which Latinas are able to experience sexual wellbeing. Intersectionality and reproductive justice support continued exploration of structural factors impacting health outcomes. Perhaps when life stressors related to income and education are strong, intimacy and pleasure with a partner(s) is a way to deal with those pressures as it is possible that sex functions as a coping mechanism. 
Number of vaginal sex partners and its correlation to sexual satisfaction is another important finding. This demonstrates that, Latinas are not "virginal" creatures as tenets of Marianísmo would suggest (Torres, Solberg \& Carlstrom, 2002) but are actively engaging in their sexual lives. Cultural narratives around hypersexuality are questioned and challenged, as Latinas in this sample are educated, have life experience, health insurance, and actively engage in sex. Additionally, and in taking a sex positive approach, having multiple experiences with several partners could help Latinas establish what their sexual and romantic preferences are, and thus why a sample of adult Latinas like this would report high levels of sexual satisfaction.

\section{Latinas, Sexual Satisfaction, Contraception and STIs}

Although I found no statistically significant correlation between sexual satisfaction and contraceptive utilization or sexual satisfaction and STI history, some insights are worth discussing. Regarding contraception specifically, Latinas are employing strategies to manage their own sexual health as condom use and oral birth control were the most reported forms of contraception use amongst all Latina ethnic subgroups. Jackson et al. (2015) found that Latinas (and I would assume most women) prefer a contraceptive method they can "control." "Control" in this instance has two meanings: "control" in that they do not need to rely on a partner for contraception and "control" related to being able to easily stop contraceptive use when they want (such as in the case of wanting to get pregnant). These may be some of the reasons Latinas in this study preferred condoms and the pill to other longer acting forms of contraception such as IUD's and the depo shot which rely on medical providers for initiation and to 
discontinuation of use. Intersectionality and reproductive justice approaches support that contraception and STI prevention methods are individualized choices for women, ones that provide power and control over their reproductive and sexual health. That being said, and considering this study's lack of statistically significant correlations between contraceptive utilization and sexual satisfaction, it appears that contraceptive use could be compartmentalized to another dimension of sexual health as it does not appear to be related to experiences of pleasure or desire for this sample of Latinas. Additionally, the withdrawal and "no method used" were the third and fourth most widely reported contraceptive strategies for women in this sample. Considering the age of women in this sample, and that many reported being in more long term/exclusive relationships with their partners, this finding is not only not surprising, it is also somewhat unremarkable. It makes sense that Latinas in committed, long term relationships where they feel considerable sense of relationship satisfaction with their partners, might not be concerned about STIs in particular, or unwanted pregnancy, because they love and trust their partners, not because Latinas are irresponsible and don't want to protect or take care of their sexual health needs.

The CDC (2016) has reported chlamydia, gonorrhea, and syphilis to be among some of the most prevalent STIs amongst Latinas/os in the US; with Latinas/os being nearly two times as likely to be diagnosed with these diseases over whites. HPV is also considerably prevalent in Latina populations and bears similar statistics, with Latinas being two times as likely as whites to be diagnosed with HPV (CDC, 2016). In the case of gonorrhea in particular, rates are staggeringly high amongst Latinas/os and are 3 times that of whites. Although overall reported rates of having ever been diagnosed with an STI 
was low amongst this sample, the top 3 reported STIs were in fact chlamydia (15\%), genital herpes (4\%) and HPV (6\%). Syphilis rates were low amongst this sample. My findings suggested no statistically significant correlation between sexual satisfaction and STIs amongst this sample; this could be due to the low reported numbers of STIs in this particular dataset. Unfortunately, I am unable to deduce the reasons for the low reported numbers of STIs and could speculate that perhaps it has something to do with lack of testing, but I am unable to know this for sure based on this dataset. However, the history of STIs reported in this sample could still be concerning, and can still suggest high exposure to "risky" sex behaviors in the past- certainly an issue faced by young people of all sociodemographic backgrounds. An implication of this would be to still promote and encourage routine HIV/STI and gynecological examinations for Latina women.

\section{Study Limitations and Directions for Future Research}

This study had numerous limitations which need to be examined and considered for any future research into this topic. To begin with, this data set is from 2008, and thus the fact that it is 10 years old is a limitation because of the social, political and economic changes that have occurred since then. Secondly, although 287 is an ample sample size (above 30-40 typically needed for statistical analysis [Field, 2013]), many of the subgroups had little representation for a robust quantitative analysis. There were only 12 women who identified as Cuban and 19 who identified as Chicana making inferential analysis with these two groups impossible. Ideally there would be at least 30-40 participants in each subgroup (Field, 2013), instead of 19 from the Chicana group or 12 from the Cubana group. Additionally, the background of the "Latina other" group is also 
unknown, although we can suspect that women from the Dominican Republic might have fit into this category. Also, sample bias is a limitation because there was some attrition from the original group. These limitations regarding small numbers for Latina subgroups makes analyses challenging if not impossible.

The purpose of the Add Health survey was not specifically to probe around issues of sexual satisfaction, and more than one question is needed to really explore this complex construct. Because this study was correlational, so the direction of influence cannot be ascertained. Moreover, other sexual behaviors associated with sexual satisfaction such as masturbation (Haavio-Mannila \& Kontula, 1997; Young \& Luquis, 1998; Litzinger \& Gordon, 2005; Barrientos \& Páez, 2006) were not assessed by the Add Health survey and also likely impact relationship and sexual satisfaction. Future research on sexual satisfaction must consider other variables which may also impact higher levels of reported sexual satisfaction for Latina women.

This particular sample was overwhelmingly represented by Latinas who identify primarily as heterosexual. There was very little representation of Latinas who identify with other sexual orientations. This is a considerable limitation of an intersectional, critical theory oriented analysis of sexuality and race. More representation of Latinas outside of heterosexual identification is needed to truly capture intersectional aspects of this topic. Additionally, sexual health needs of transgender women should also be explored, and this study did not include or address women who identify as transgender.

This study also did not collect or analyze data related to participants' experiences with racism and discrimination, despite knowing that people of color experience oppression (structurally and on a micro level) on a daily basis and that there are real life 
health consequences to such experiences. Further work should investigate Latinas' experiences with racism and discrimination as they affect sexual health. As these findings illustrate, when Latinas have access to education, employment, health insurance, they also report being sexually satisfied, which is considered a positive sexual health outcome. A hypothesis for future study is that if Latinas were also free of experiences of racism and discrimination, their sexual health would only be that much better.

Implications for Social Work Practice, Research and Education

This has numerous implications for social work practice, research and education. Latina critical perspectives are lacking in social work: sexual health is often overlooked or approached from a deficit based framework (Dodd \& Tolman, 2017; McCave, Shepard \& Winter, 2014) and this study offers an example of a sex positive, critical and intersectional investigation. Findings from this study on correlates of sexual satisfaction have the potential to inform sexual health and education with all populations social work cares about. This study contributes to our knowledge of Latina sexual health from an explicitly social work lens. A growing body of scholarship has indicated that positive sexual experiences are strongly associated with more traditional sexual health outcomes such as the ability to protect oneself from disease and unplanned pregnancy (Higgins et al., 2011). Sexual health promote on efforts must consider explicitly supporting healthy relationships within Latinx populations. For Latina women in heterosexual relationships specifically, health promotion programs should be designed to enhance interpersonal relationships based on mutual trust, respect and care from a culturally relevant approach. This study shows that quality relationships improve sexual satisfaction, which in turn 
could improve overall health (Stephenson \& Meston, 2015). I also think it is important to consider that the definition of what is a "healthy relationship" must be defined by members of Latina communities, as white centric definitions of this concept might prove to be culturally irrelevant to Latina groups.

In terms of social work practice, many social work practitioners will be working with Latinx service users in some capacity. Social workers will be working with service users impacted by sexual health issues and needs. Understanding the dynamic forces at play regarding sexual health for Latina populations is necessary for culturally relevant health promotion. Additionally, social work practitioners are also actively engaged in policy work, and can advocate for social policies which promote and advance sexual health of all populations. People have a right to experience sexual pleasure, yet people can only make these choices freely when they have access to current, evidence based and comprehensive sexuality education and a responsive health care system where they are free from structural forms of oppression. Social workers as policy advocates can continue to support health care reform and access, access to contraception and abortion, as well as comprehensive sex education, since issues related to sexuality are shaped by the social and political milieu in which we operate.

Regarding social work research, this study shows the potential utility of applying critical and intersectional theorizing to quantitative methods, effectively combining critical epistemologies with quantitative methods in the pursuit of social justice focused research. Intersectionality and critical theory are popular theoretical orientations in the social work discipline, and are used with some regularity in practice. This project could specifically inform variable selection and how to use specific statistical tests under the 
auspices of an intersectional and critical theory approach. Also, I make the case for social work having more of a presence in sexual health scholarship; specifically, that social work can and should make contributions to this particular field instead of continuing to rely on the work of other professions, especially considering the explicitly social justice and anti-oppressive nature of social work. We are well suited to contribute to the growing body of scholarship on sexual health (Dodd \& Tolman, 2017). This study is an example of the potential for strengths based approaches nested in sex positive frameworks to inform careful considerations of interpretations of findings when conducting sexuality related research with communities of color. It was also my hope that by drawing attention to an understudied component of sexual health, I can contribute to useful guidance on health promotion. González-Lopez \& Vidal-Ortiz (2008) have asked the question, can we conduct sociological quantitative and qualitative studies that foreground Latinas' experiences with sexuality that move beyond the usual topics (e.g. bearing of children, economic hardship linked to the former, health concerns, sexual victimization) while not erasing them altogether and further develop others (e.g. sexual pleasure, identity, and sexuality among Latinas women of all sexual orientations, sexuality and racialization)? (p.316)

I believe this is not only urgently necessary because of our political and social reality, but also possible as this study attempts to capture and address exactly what Gónzalez-López \& Vidal-Ortiz (2008) are imploring of social scientists. This study is a step in this direction despite its many limitations. Beyond addressing the "usual topics," I am exploring other understudied dimensions of sexual health as described by Bowleg (2012):

far from being just an exercise in semantics, intersectionality provides the discipline of public health with a critical unifying interpretive and analytical framework for reframing how public health scholars conceptualize, investigate, analyze, and address disparities and social inequality in health (p.1267) 
Similarly, LatCrit has the potential to "center the margins" by challenging commonly held beliefs about Latinas, sexual health and sexuality (Ford \& Arihihenbuwa, 2010) to consider overlooked and understudied dimensions about Latina culture and sexual health disparities.

Despite a growing interest by social workers in sexual health promotion, this content is not yet required at any level of social work education (CSWE, 2015). Considerable variance exists across social work programs in the US regarding the inclusion of content related to sexual health (Winter et al., 2016). In many instances, students may never have exposure to this material, yet, as previously mentioned, are likely to be working with individuals and families who may experience adverse outcomes related to sexual health (Winter et al., 2016). The extent to which social workers are exposed to nuanced content about the Latina/o condition also varies considerably across BSW and MSW programs in the US, with some programs being more intentional about the inclusion of this material than others. However, content related to intersectionality and critical theorizing is present in many social work curriculums. Social work, with its emphasis on engaging with human development through the lifespan is well positioned to continue to critically engage with issues of sexual health because "by recognizing sexuality as an integral part of social work education and practice, we can replace patterns of paternalism with strengths based models of care more consistent" with social work's code of ethics and evidence based practice (McCave, Shepard \& Winter, 2017, p.321). 


\section{Conclusion}

This study answered the four research questions. The strongest correlates of sexual satisfaction are relationship factors for Latinas in this sample and there were no significant group differences amongst the various Latina subgroups with regards to sexual satisfaction. Contraception use and STI history were not correlated with sexual satisfaction.

This study aligns well with the values and ethics of the social work profession. Historical social work approaches may have been heavily informed by paternalistic attitudes towards communities of color, and this study, with its emphasis on challenging stereotypes, is evidence of a person centered, strengths based approach currently embraced by the social work profession (NASW, 2015). It is also really important that this study about Latina sexual health, although informed by other disciplines, is explicitly nested in social work as it has numerous implications for social work practice, research and education. It also builds off of the work from many feminist scholars of color who have made such important contributions to strengths based, and culturally relevant scholarship. The critical theoretical orientations informing this study encourage challenging stereotypes of Latina sexuality which can inform health promotion and policy advocacy efforts for social workers working with Latinx populations. 


\section{TABLES}

Table 1. Demographic Characteristics

\begin{tabular}{|c|c|c|c|c|c|c|c|c|c|c|c|c|c|c|}
\hline \multirow{2}{*}{$\begin{array}{l}\text { Characteristic } \\
\text { Multi-racial } \\
\text { identity }\end{array}$} & \multicolumn{2}{|c|}{$\begin{array}{c}\text { Total } \\
(\mathrm{n}=287)\end{array}$} & \multicolumn{2}{|c|}{$\begin{array}{l}\text { Mexican } \\
(\mathrm{n}=161)\end{array}$} & \multicolumn{2}{|c|}{$\begin{array}{l}\text { Puerto Rican } \\
\quad(\mathrm{n}=39)\end{array}$} & \multicolumn{2}{|c|}{$\begin{array}{c}\text { Central \& South } \\
\text { American }(\mathrm{n}=33)\end{array}$} & \multicolumn{2}{|c|}{ Chicana $(\mathrm{n}=19)$} & \multicolumn{2}{|c|}{ Cuban $(\mathrm{n}=12)$} & \multicolumn{2}{|c|}{$\begin{array}{l}\text { Hispanic Other } \\
\qquad(\mathrm{n}=37)\end{array}$} \\
\hline & $\mathrm{n}$ & $\%$ & $\mathrm{n}$ & $\%$ & $\mathrm{n}$ & $\%$ & $\mathrm{n}$ & $\%$ & $\mathrm{n}$ & $\%$ & $\mathrm{n}$ & $\%$ & $\mathrm{n}$ & $\%$ \\
\hline $\begin{array}{l}\text { Latina \& } \\
\text { Black }\end{array}$ & 17 & 5.9 & 6 & 3.7 & 7 & 17.9 & - & - & - & - & 2 & 16.7 & 3 & 8.1 \\
\hline $\begin{array}{l}\text { Latina \& } \\
\text { Native } \\
\text { American }\end{array}$ & 23 & 8 & 18 & 11.2 & 2 & 5.1 & 2 & 6.1 & 2 & 10.5 & - & - & - & - \\
\hline $\begin{array}{l}\text { Latina \& } \\
\text { Asian }\end{array}$ & 6 & 2.1 & 1 & 0.6 & - & - & - & - & - & - & - & - & 5 & 13.5 \\
\hline $\begin{array}{l}\text { Latina, Asian } \\
\text { \& Black }\end{array}$ & 1 & 0.3 & - & - & - & - & - & - & - & - & - & - & 1 & 2.7 \\
\hline Sexual & M & SD & M & $\mathrm{SD}$ & M & $\mathrm{SD}$ & M & SD & M & SD & $\mathrm{M}$ & $\mathrm{SD}$ & M & $\mathrm{SD}$ \\
\hline (continuous) & 1.83 & 1.06 & 1.83 & 1.08 & 1.81 & 1.09 & 1.94 & 1.08 & 1.68 & 0.67 & 1.92 & 1.16 & 1.82 & 1.17 \\
\hline $\begin{array}{l}\text { Sexual } \\
\text { Satisfaction }\end{array}$ & $\mathrm{n}$ & $\%$ & $\mathrm{n}$ & $\%$ & $\mathrm{n}$ & $\%$ & $\mathrm{n}$ & $\%$ & $\mathrm{n}$ & $\%$ & $\mathrm{n}$ & $\%$ & $\mathrm{n}$ & $\%$ \\
\hline Agree & 230 & 80.1 & 130 & 80.7 & 30 & 77 & 27 & 81.9 & 17 & 89.5 & 10 & 83.4 & 28 & 75.6 \\
\hline $\begin{array}{l}\text { Neither agree } \\
\text { nor disagree }\end{array}$ & 19 & 6.6 & 9 & 5.6 & 3 & 7.7 & 2 & 6.1 & 2 & 10.5 & 1 & 8.3 & 2 & 5.4 \\
\hline Disagree & 27 & 9.4 & 18 & 11.2 & 3 & 7.7 & 3 & 9.1 & 0 & 0 & 1 & 8.3 & 4 & 10.8 \\
\hline \multicolumn{15}{|l|}{ Age } \\
\hline $35-39$ & 180 & 58.2 & 98 & 55.9 & 25 & 58.9 & 23 & 58.9 & 13 & 63.2 & 4 & 16.6 & 23 & 67.5 \\
\hline $40-45$ & 107 & 41.8 & 63 & 44.1 & 14 & 41 & 10 & 41 & 6 & 36.9 & 8 & 83.3 & 14 & 32.4 \\
\hline
\end{tabular}


Table 1

Continued

\begin{tabular}{|c|c|c|c|c|c|c|c|c|c|c|c|c|c|c|}
\hline \multicolumn{15}{|l|}{ Education } \\
\hline $\begin{array}{l}\text { Less than high } \\
\text { school }\end{array}$ & 28 & 9.7 & 15 & 9.3 & 5 & 12.8 & 3 & 9.1 & 2 & 10.5 & 0 & 0 & 2 & 5.4 \\
\hline $\begin{array}{l}\text { High School } \\
\text { graduate }\end{array}$ & 53 & 18.5 & 37 & 23 & 6 & 15.4 & 6 & 18.2 & 0 & 0 & 0 & 0 & 4 & 10.8 \\
\hline $\begin{array}{l}\text { Vocational } \\
\text { Training }\end{array}$ & 38 & 13.2 & 22 & 13.7 & 7 & 18 & 3 & 9.1 & 3 & 15.8 & 1 & 8.3 & 3 & 8.1 \\
\hline Some college & 97 & 33.8 & 59 & 36.6 & 13 & 33.3 & 13 & 33.3 & 10 & 52.6 & 4 & 33.3 & 9 & 24.3 \\
\hline $\begin{array}{l}\text { Bachelor's } \\
\text { degree or } \\
\text { higher }\end{array}$ & 71 & 24.7 & 28 & 17.3 & 8 & 20.5 & 8 & 20.5 & 4 & 21.1 & 7 & 58.3 & 19 & 51.3 \\
\hline \multicolumn{15}{|c|}{ Annual Household Income } \\
\hline $\begin{array}{l}\text { Less than } \\
\$ 25,000\end{array}$ & 39 & 13.5 & 19 & 11.7 & 10 & 25.6 & 6 & 18.3 & 2 & 10.6 & 0 & 0 & 2 & 5.4 \\
\hline $\begin{array}{l}\$ 25,000- \\
\$ 49,000\end{array}$ & 87 & 30.3 & 58 & 36 & 10 & 25.6 & 7 & 21.3 & 3 & 15.9 & 1 & 8.3 & 10 & 27 \\
\hline Over $\$ 50,000$ & 130 & 45.3 & 64 & 39.7 & 13 & 33.4 & 19 & 57.6 & 11 & 57.9 & 10 & 58.3 & 22 & 40.5 \\
\hline \multicolumn{15}{|c|}{ Health Insurance Status } \\
\hline $\begin{array}{l}\text { Yes Health } \\
\text { Insurance }\end{array}$ & 191 & 66.4 & 102 & 63.4 & 22 & 56.5 & 22 & 66.6 & 15 & 79 & 12 & 100 & 31 & 83.8 \\
\hline $\begin{array}{l}\text { No Health } \\
\text { Insurance }\end{array}$ & 50 & 17.4 & 34 & 21.1 & 5 & 12.8 & 8 & 24.2 & 1 & 5.3 & - & - & 3 & 8.1 \\
\hline Medicaid & 38 & 13.2 & 21 & 13 & 10 & 25.6 & 3 & 9.1 & 2 & 10.5 & - & - & 2 & 5.4 \\
\hline Don't Know & 8 & 2.8 & 1 & 2.7 & 2 & 5.1 & - & - & 1 & 5.3 & - & - & 1 & 2.7 \\
\hline
\end{tabular}


Table 1

Continued

\section{US Citizenship}

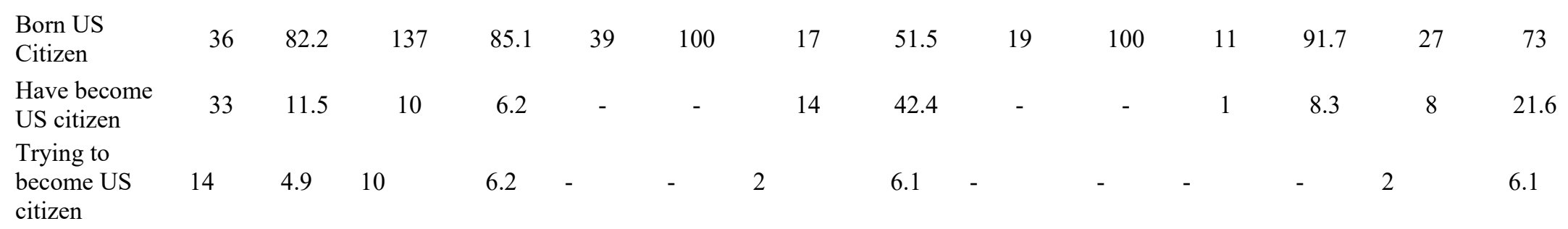

Language Used

Most at Home

\begin{tabular}{|c|c|c|c|c|c|c|c|c|c|c|c|c|c|c|}
\hline English & 98 & 34.1 & 51 & 31.7 & 13 & 33.3 & 12 & 36.4 & 13 & 68.4 & 3 & 25 & 15 & 40.5 \\
\hline Spanish & 76 & 25.5 & 42 & 26.1 & 6 & 15.4 & 15 & 45.5 & 2 & 10.5 & 8 & 66.7 & 6 & 16.2 \\
\hline \multicolumn{15}{|c|}{ Sexual Orientation } \\
\hline Heterosexual & 228 & 79.4 & 128 & 79.5 & 28 & 71.8 & 27 & 81.8 & 16 & 84.2 & 10 & 83.3 & 31 & 83.8 \\
\hline $\begin{array}{l}\text { Mostly } \\
\text { Heterosexual }\end{array}$ & 38 & 13.2 & 22 & 13.7 & 6 & 15.4 & 5 & 15.2 & 3 & 15.8 & 1 & 8.3 & 4 & 10.8 \\
\hline Bisexual & 9 & 3.1 & 3 & 1.9 & 4 & 10.3 & 0 & 0 & 0 & 0 & 1 & 8.3 & 0 & 0 \\
\hline $\begin{array}{l}\text { Mostly } \\
\text { Homosexual }\end{array}$ & 4 & 1.4 & 3 & 1.9 & 1 & 2.6 & 0 & 0 & 0 & 0 & 0 & 0 & 0 & 0 \\
\hline Homosexual & 2 & 0.7 & 1 & 0.6 & 0 & 0 & 1 & 3 & 0 & 0 & 0 & 0 & 0 & 0 \\
\hline
\end{tabular}


Table

\begin{tabular}{|c|c|c|c|c|c|c|c|c|c|c|c|c|c|c|}
\hline \multicolumn{15}{|l|}{ Continued } \\
\hline $\begin{array}{l}\text { Not attracted } \\
\text { to males or } \\
\text { females }\end{array}$ & 1 & 0.33 & 1 & 0.6 & 0 & 0 & 0 & 0 & 0 & 0 & 0 & 0 & 0 & 0 \\
\hline \multicolumn{15}{|c|}{ Relationship Status } \\
\hline Engaged & 5 & 1.7 & 4 & 2.5 & 0 & 0 & 0 & 0 & 0 & 0 & 1 & 8.3 & 0 & 0 \\
\hline $\begin{array}{l}\text { Dating } \\
\text { exclusively }\end{array}$ & 32 & 11.1 & 15 & 9.3 & 4 & 10.3 & 3 & 9.1 & 2 & 10.5 & 1 & 8.3 & 8 & 21.6 \\
\hline $\begin{array}{l}\text { Dating } \\
\text { frequently, } \\
\text { but not } \\
\text { exclusively }\end{array}$ & 8 & 2.8 & 6 & 3.7 & 1 & 2.6 & 1 & 3 & 0 & 0 & 0 & 0 & 1 & 2.7 \\
\hline $\begin{array}{l}\text { Dating once } \\
\text { in a while }\end{array}$ & 4 & 1.4 & 3 & 1.9 & 2 & 5.1 & 0 & 0 & 0 & 0 & 0 & 0 & 0 & 0 \\
\hline $\begin{array}{l}\text { Only having } \\
\text { sex }\end{array}$ & 8 & 2.8 & 6 & 3.7 & 0 & 0 & 2 & 6.1 & 0 & 0 & 0 & 0 & 0 & 0 \\
\hline $\begin{array}{l}\text { Relationship } \\
\text { Satisfaction }\end{array}$ & $\mathrm{M}$ & SD & M & $\mathrm{SD}$ & M & $\mathrm{SD}$ & M & $\mathrm{SD}$ & $\mathrm{M}$ & $\mathrm{SD}$ & $\mathrm{M}$ & SD & $\mathrm{M}$ & $\mathrm{SD}$ \\
\hline (continuous) & 1.82 & 0.87 & 1.81 & 0.87 & 1.84 & 0.87 & 2.10 & 0.96 & 1.68 & 0.59 & 1.54 & 0.86 & 1.65 & 0.81 \\
\hline $\begin{array}{l}\text { Enjoy ordinary } \\
\text { things together }\end{array}$ & $\mathrm{n}$ & $\%$ & $\mathrm{n}$ & $\%$ & $\mathrm{n}$ & $\%$ & $\mathrm{n}$ & $\%$ & $\mathrm{n}$ & $\%$ & $\mathrm{n}$ & $\%$ & $\mathrm{n}$ & $\%$ \\
\hline Agree & 234 & 81.5 & 133 & 82.6 & 29 & 74.4 & 26 & 78.8 & 18 & 94.7 & 11 & 91.6 & 30 & 81.1 \\
\hline $\begin{array}{l}\text { Neither agree } \\
\text { nor disagree }\end{array}$ & 25 & 8.7 & 14 & 8.7 & 4 & 10.3 & 3 & 9.1 & 1 & 5.3 & 0 & 0 & 2 & 5.4 \\
\hline Disagree & 20 & 6.9 & 11 & 6.8 & 3 & 7.7 & 2 & 6.1 & 0 & 0 & 1 & 8.3 & 3 & 8.1 \\
\hline
\end{tabular}


Table 1

Continued

Satisfied handling problems with partner

\begin{tabular}{|c|c|c|c|c|c|c|c|c|c|c|c|c|c|c|}
\hline Agree & 199 & 69.4 & 118 & 73.3 & 24 & 61.5 & 20 & 60.6 & 15 & 79 & 9 & 75 & 25 & 67.5 \\
\hline $\begin{array}{l}\text { Neither agree } \\
\text { nor disagree }\end{array}$ & 31 & 10.8 & 14 & 8.7 & 3 & 7.7 & 5 & 15.2 & 2 & 10.5 & 2 & 16.7 & 5 & 13.5 \\
\hline Disagree & 49 & 17.1 & 26 & 16.1 & 9 & 23.1 & 5 & 15.2 & 1 & 5.3 & 1 & 8.3 & 5 & 13.5 \\
\hline
\end{tabular}

Satisfied handling finances with partner

$\begin{array}{lcccccccccrrrrrr}\text { Agree } & 163 & 56.8 & 84 & 52.2 & 24 & 61.6 & 18 & 54.5 & 12 & 57.9 & 8 & 66.6 & 26 & 70.2 \\ \begin{array}{l}\text { Neither agree } \\ \text { nor disagree }\end{array} & 61 & 21.3 & 38 & 23.6 & 7 & 17.9 & 8 & 24.2 & 5 & 26.3 & 2 & 16.7 & 7 & 18.9 \\ \text { Disagree } & 56 & 19.2 & 36 & 22.4 & 5 & 12.8 & 5 & 15.2 & 3 & 15.8 & 2 & 16.7 & 2 & 5.4\end{array}$

Partner listens when need to talk

\begin{tabular}{|c|c|c|c|c|c|c|c|c|c|c|c|c|c|c|}
\hline Agree & 213 & 74.2 & 120 & 74.6 & 30 & 77 & 21 & 63.6 & 17 & 89.5 & 10 & 83.3 & 27 & 73 \\
\hline $\begin{array}{l}\text { Neither agree } \\
\text { nor disagree }\end{array}$ & 31 & 10.8 & 16 & 9.9 & 2 & 5.1 & 7 & 21.2 & 1 & 5.3 & 1 & 8.3 & 5 & 13.5 \\
\hline Disagree & 35 & 12.2 & 22 & 13.7 & 4 & 10.2 & 4 & 12.1 & 1 & 5.3 & 1 & 8.3 & 3 & 8.1 \\
\hline
\end{tabular}

Partner expresses love and affection

\begin{tabular}{|c|c|c|c|c|c|c|c|c|c|c|c|c|c|}
\hline Agree & 231 & 80.5 & 131 & 81.4 & 29 & 74.3 & 26 & 78.8 & 16 & 84.2 & 11 & 91.7 & 32 \\
\hline $\begin{array}{l}\text { Neither agree } \\
\text { nor disagree }\end{array}$ & 26 & 9.1 & 15 & 9.3 & 4 & 10.3 & 2 & 6.1 & 3 & 15.8 & 1 & 8.3 & 2 \\
\hline
\end{tabular}


Table 1

Continued

$\begin{array}{llllllllllllllllllll}\text { Disagree } & 22 & 7.7 & 12 & 7.4 & 3 & 7.7 & 2 & 6.1 & 0 & 0 & 0 & 0 & 1 & 2.7\end{array}$

Trust partner to be faithful

$\begin{array}{lccccccccccrrrrr}\text { Agree } & 212 & 73.8 & 125 & 77.6 & 28 & 71.8 & 18 & 54.5 & 14 & 73.7 & 10 & 83.4 & 29 & 78.4 \\ \begin{array}{l}\text { Neither agree } \\ \text { nor disagree }\end{array} & 29 & 10.1 & 14 & 8.7 & 3 & 7.7 & 8 & 24.2 & 2 & 10.5 & 1 & 8.3 & 4 & 10.8 \\ \text { Disagree } & 38 & 13.2 & 19 & 11.8 & 5 & 12.8 & 5 & 15.2 & 2 & 10.5 & 1 & 8.3 & 2\end{array}$

Extent participant loves partner

\begin{tabular}{|c|c|c|c|c|c|c|c|c|c|c|c|c|c|c|}
\hline A lot & 206 & 71.8 & 118 & 73.3 & 23 & 59 & 18 & 54.5 & 18 & 94.7 & 9 & 75 & 28 & 75.7 \\
\hline Somewhat & 16 & 5.6 & 8 & 5 & 4 & 10.3 & 4 & 12.1 & 0 & 0 & 0 & 0 & 1 & 2.7 \\
\hline A little & 10 & 3.5 & 5 & 3.1 & 0 & 0 & 1 & 3 & 0 & 0 & 1 & 8.3 & 3 & 8.1 \\
\hline Not at all & 8 & 2.8 & 4 & 2.5 & 1 & 2.6 & 3 & 9.1 & 0 & 0 & 0 & 0 & 1 & 2.7 \\
\hline
\end{tabular}

Extent participant is happy with partner

\begin{tabular}{|c|c|c|c|c|c|c|c|c|c|c|c|c|c|c|}
\hline Very & 171 & 59.6 & 103 & 64 & 18 & 46.2 & 12 & 36.4 & 13 & 68.4 & 7 & 58.3 & 25 & 67.6 \\
\hline Fairly & 51 & 17.8 & 25 & 15.5 & 9 & 23.1 & 9 & 27.3 & 5 & 26.3 & 1 & 8.3 & 5 & 13.5 \\
\hline Not too happy & 18 & 6.3 & 7 & 4.3 & 1 & 2.6 & 5 & 15.2 & 0 & 0 & 2 & 16.7 & 3 & 8.1 \\
\hline
\end{tabular}


Table 1

Continued

Extent participant is committed to partner

\begin{tabular}{|c|c|c|c|c|c|c|c|c|c|c|c|c|c|c|}
\hline Completely & 163 & 56.8 & 92 & 57.1 & 19 & 48.7 & 11 & 33.3 & 14 & 73.7 & 8 & 66.7 & 25 & 67.6 \\
\hline Very & 47 & 16.4 & 26 & 16.1 & 5 & 12.8 & 10 & 30.3 & 4 & 21.1 & 1 & 8.3 & 3 & 8.1 \\
\hline Somewhat & 14 & 4.9 & 7 & 4.3 & 3 & 7.7 & 3 & 9.1 & 0 & 0 & 0 & 0 & 2 & 5.4 \\
\hline Not at all & 16 & 5.6 & 10 & 6.2 & 1 & 2.6 & 2 & 6.1 & 0 & 0 & 1 & 8.3 & 3 & 8.1 \\
\hline
\end{tabular}

Contraceptive Use Past 12 months

\begin{tabular}{|c|c|c|c|c|c|c|c|c|c|c|c|c|c|c|}
\hline Condom & 103 & 35.9 & 55 & 34.2 & 19 & 48.7 & 13 & 39.4 & 6 & 31.6 & 5 & 41.7 & 11 & 29.7 \\
\hline $\begin{array}{l}\text { Oral Birth } \\
\text { Control }\end{array}$ & 79 & 27.5 & 41 & 25.5 & 11 & 28.2 & 8 & 24.2 & 7 & 36.8 & 5 & 41.7 & 13 & 35.1 \\
\hline $\begin{array}{l}\text { Birth Control } \\
\text { Injection }\end{array}$ & 6 & 2.1 & 3 & 1.9 & 2 & 5.1 & 1 & 3 & 1 & 5.3 & - & - & 1 & 2.7 \\
\hline $\begin{array}{l}\text { Emergency } \\
\text { Contraception }\end{array}$ & 4 & 1.4 & 3 & 1.9 & 2 & 5.1 & 1 & 3 & - & - & - & - & - & - \\
\hline IUD & 11 & 3.8 & 8 & 5 & - & - & 2 & 6.1 & - & - & 1 & 8.3 & - & - \\
\hline $\begin{array}{l}\text { Withdrawal } \\
\text { Method }\end{array}$ & 36 & 12.5 & 20 & 12.4 & 7 & 17.9 & 5 & 15.2 & 1 & 5.3 & 1 & 8.3 & 4 & 10.8 \\
\hline $\begin{array}{l}\text { Rhythm } \\
\text { Method }\end{array}$ & 6 & 2.1 & 3 & 1.9 & 2 & 5.1 & - & - & 1 & 5.3 & 1 & 8.3 & - & - \\
\hline Ring & 5 & 1.7 & 3 & 1.9 & 1 & 2.6 & 7 & 21.2 & 2 & 10.5 & - & - & - & - \\
\hline $\begin{array}{l}\text { Birth Control } \\
\text { Patch }\end{array}$ & 4 & 1.4 & 1 & 0.6 & 1 & 2.6 & 2 & 6.1 & 2 & 10.5 & - & - & - & - \\
\hline
\end{tabular}


Table 1

\begin{tabular}{|c|c|c|c|c|c|c|c|c|c|c|c|c|c|c|}
\hline \multicolumn{15}{|l|}{ Continued } \\
\hline $\begin{array}{l}\text { Tubal } \\
\text { Ligation }\end{array}$ & 10 & 3.5 & 4 & 2.5 & 3 & 7.7 & 1 & 3 & 1 & 5.3 & 1 & 8.3 & 1 & 2.7 \\
\hline $\begin{array}{l}\text { No Method } \\
\text { Used }\end{array}$ & 50 & 17.4 & 31 & 19.3 & 9 & 23.1 & 4 & 12.1 & 3 & 15.8 & 3 & 25 & 3 & 8.1 \\
\hline \multicolumn{15}{|c|}{ Ever Diagnosed w/ STI/STD } \\
\hline Chlamydia & 42 & 14.6 & 30 & 18.6 & 5 & 12.8 & 1 & 3 & 6 & 31.6 & - & - & 3 & 8.1 \\
\hline Gonorrhea & 6 & 2.1 & 2 & 1.2 & 2 & 5.1 & 1 & 3 & 1 & 5.3 & - & - & - & - \\
\hline Syphilis & 1 & 0.3 & 1 & 0.6 & - & - & - & - & - & - & - & - & - & - \\
\hline $\begin{array}{l}\text { Genital } \\
\text { Herpes }\end{array}$ & 12 & 4.2 & 5 & 3.1 & 3 & 7.7 & 1 & 3 & 3 & 15.8 & - & - & - & - \\
\hline Genital Warts & 10 & 3.5 & 9 & 5.6 & - & - & - & - & 1 & 5.3 & - & - & 1 & 2.7 \\
\hline Hep B & 6 & 2.1 & - & - & - & - & - & - & - & - & - & - & - & - \\
\hline HPV & 17 & 5.9 & 11 & 6.8 & 1 & 2.6 & 1 & 3 & - & - & 1 & 8.3 & 3 & 8.1 \\
\hline
\end{tabular}

$\bar{\perp}$ 
Table 2. Correlations between Sexual Satisfaction and Socioeconomic Factors

\begin{tabular}{|c|c|c|c|c|c|c|c|c|c|c|c|c|c|}
\hline Measure & 1 & 2 & 3 & 4 & 5 & 6 & 7 & 8 & 9 & 10 & 11 & 12 & 13 \\
\hline $\begin{array}{l}\text { 1. Sexual } \\
\text { satisfaction }\end{array}$ & - & & & & & & & & & & & & \\
\hline 2. Age & -0.09 & - & & & & & & & & & & & \\
\hline $\begin{array}{l}\text { 3. Language used } \\
\text { with family }\end{array}$ & 0.03 & 0.06 & - & & & & & & & & & & \\
\hline $\begin{array}{l}\text { 4. Speak/write } \\
\text { Spanish }\end{array}$ & 0.04 & $.18^{-}$ & -0.01 & - & & & & & & & & & \\
\hline $\begin{array}{l}\text { 5. Born a US } \\
\text { citizen }\end{array}$ & -0.06 & 0.11 & $.31^{* *}$ & $.27^{* *}$ & - & & & & & & & & \\
\hline $\begin{array}{l}\text { 6. Have become a } \\
\text { US citizen }\end{array}$ & -0.07 & -0.05 & 0.01 & -0.14 & . & - & & & & & & & \\
\hline $\begin{array}{l}\text { 7. Trying to } \\
\text { become a US } \\
\text { citizen }\end{array}$ & -0.06 & -0.07 & -0.05 & -0.13 & . & . & - & & & & & & \\
\hline $\begin{array}{l}\text { 8. High school } \\
\text { graduation status }\end{array}$ & -0.03 & $.14^{*}$ & 0.00 & -0.11 & -0.01 & 0.06 & -0.10 & - & & & & & \\
\hline $\begin{array}{l}\text { 9. Highest } \\
\text { education level }\end{array}$ & 0.05 & $-.12 *$ & -0.07 & 0.08 & -0.06 & $.31 *$ & -0.15 & $-4^{-}$ & - & & & & \\
\hline $\begin{array}{l}\text { 10. Total household } \\
\text { income }\end{array}$ & -0.03 & -0.12 & -0.05 & 0.03 & 0.03 & $.37 *$ & 0.14 & $.25^{* *}$ & $.37 * *$ & - & & & \\
\hline 11. Present religion & 0.04 & -0.04 & 0.02 & -0.02 & 0.04 & 0.05 & 0.16 & 0.06 & -0.10 & -0.09 & - & & \\
\hline $\begin{array}{l}\text { 12. Importance of } \\
\text { religion }\end{array}$ & -0.09 & -0.05 & -0.01 & 0.08 & -0.02 & 0.06 & 0.16 & -0.02 & 0.04 & -0.03 & $.34 * *$ & - & \\
\hline $\begin{array}{l}\text { 13. Current health } \\
\text { insurance status }\end{array}$ & 0.00 & -0.05 & -0.02 & -0.11 & 0.01 & 0.02 & -0.05 & 0.09 & -0.06 & -0.03 & 0.03 & 0.01 & - \\
\hline
\end{tabular}

${ }^{*} p<0.05 .{ }^{*}{ }^{*} p<0.01$. 
Table 3. Correlations between Sexual Satisfaction and Reproductive Health Variables

\begin{tabular}{|c|c|c|c|c|c|c|c|}
\hline Measure & 1 & 2 & 3 & 4 & 5 & 6 & 7 \\
\hline 1. Sexual satisfaction & - & & & & & & \\
\hline 2. Sexual orientation & 0.05 & - & & & & & \\
\hline 3. Number of vaginal sex partners & 0.04 & $0.30 * *$ & - & & & & \\
\hline 4. Past 12-month condom use & 0.05 & 0.04 & -0.05 & - & & & \\
\hline 5. Past 12-month oral birth control use & -0.09 & $-0.19 * *$ & $-0.15^{*}$ & 0.05 & - & & \\
\hline 6. Past 12-month birth control injection use & -0.06 & -0.02 & $0.14^{*}$ & 0.07 & 0.05 & - & \\
\hline 7. Past 12-month emergency contraception use & 0.00 & 0.01 & $0.14^{*}$ & 0.08 & -0.03 & -0.02 & - \\
\hline 8. Past 12-month IUD use & -0.10 & -0.02 & 0.03 & -0.08 & $-0.16^{*}$ & -0.04 & -0.03 \\
\hline 9. Past 12-month withdrawal method use & -0.04 & 0.02 & 0.05 & $0.17 *$ & 0.04 & 0.08 & $0.22 * *$ \\
\hline 10. Past 12 -month tubal ligation & -0.06 & -0.06 & -0.04 & $-0.15^{*}$ & -0.06 & -0.04 & -0.03 \\
\hline 11. Past 12-month no method use & 0.01 & 0.10 & $0.13^{*}$ & $-0.48 * *$ & $-0.38 * *$ & -0.09 & -0.07 \\
\hline 12. Ever diagnosed: chlamydia & -0.06 & $0.12 *$ & $0.19 * *$ & -0.01 & $-0.14 *$ & 0.08 & 0.04 \\
\hline 13. Ever diagnosed: gonorrhea & 0.04 & 0.06 & $0.15^{*}$ & -0.01 & -0.05 & -0.03 & -0.02 \\
\hline 14. Ever diagnosed: syphilis & 0.10 & -0.03 & -0.01 & -0.06 & -0.05 & -0.01 & -0.01 \\
\hline 15. Ever diagnosed: genital herpes & 0.03 & -0.06 & 0.09 & -0.09 & -0.01 & -0.04 & -0.03 \\
\hline 16. Ever diagnosed: genital warts & -0.07 & 0.00 & $0.13^{*}$ & 0.05 & $-0.13 *$ & -0.03 & $0.17 *$ \\
\hline 17. Ever diagnosed: HPV & -0.04 & 0.06 & 0.07 & -0.08 & 0.07 & 0.06 & $0.22 * *$ \\
\hline 18. Non-physical forced sex & 0.07 & $0.16^{* *}$ & $0.32 * *$ & -0.07 & $-0.14 *$ & -0.02 & 0.09 \\
\hline 19. Physical forced sex & 0.01 & $0.14 *$ & $0.24 * *$ & -0.05 & -0.05 & -0.07 & 0.03 \\
\hline
\end{tabular}

${ }^{*} p<0.05$. ${ }^{* *} p<0.01$. 
Table 3

Continued

\begin{tabular}{|c|c|c|c|c|c|c|c|c|c|}
\hline Measure & 8 & 9 & 10 & 11 & 12 & 13 & 14 & 15 & 16 \\
\hline 8. Past 12-month IUD use & - & & & & & & & & \\
\hline 9. Past 12 -month withdrawal method use & -0.04 & - & & & & & & & \\
\hline 10. Past 12-month tubal ligation & -0.05 & -0.03 & - & & & & & & \\
\hline 11. Past 12 -month no method use & -0.12 & $-0.23 * *$ & -0.11 & - & & & & & \\
\hline 12. Ever diagnosed: chlamydia & -0.04 & -0.02 & -0.09 & 0.10 & - & & & & \\
\hline 13. Ever diagnosed: gonorrhea & 0.11 & -0.07 & -0.03 & 0.07 & 0.08 & - & & & \\
\hline 14. Ever diagnosed: syphilis & -0.02 & -0.03 & -0.01 & 0.13 & -0.03 & -0.01 & - & & \\
\hline 15. Ever diagnosed: genital herpes & -0.05 & -0.10 & 0.05 & 0.11 & 0.01 & 0.09 & -0.013 & - & \\
\hline 16. Ever diagnosed: genital warts & 0.08 & 0.06 & -0.04 & 0.09 & -0.03 & -0.03 & -0.012 & 0.054 & - \\
\hline 17. Ever diagnosed: HPV & 0.09 & 0.01 & 0.02 & -0.03 & 0.10 & -0.04 & -0.015 & -0.054 & 0.031 \\
\hline 18. Non-physical forced sex & 0.03 & 0.06 & -0.01 & 0.05 & 0.05 & -0.01 & $0.12 *$ & -0.015 & $0.20 * *$ \\
\hline 19. Physical forced sex & 0.06 & -0.03 & -0.04 & 0.05 & 0.07 & 0.07 & -0.026 & -0.043 & 0.024 \\
\hline
\end{tabular}

${ }^{*} p<0.05$. ${ }^{* *} p<0.01$.

Table 3

Continued

\begin{tabular}{lccc}
\multicolumn{1}{c}{ Measure } & 17 & 18 & 19 \\
\hline 17. Ever diagnosed: HPV & - & & - \\
18. Non-physical forced sex & $0.14^{*}$ & $0.66^{* *}$ & - \\
19. Physical forced sex & 0.056 & & \\
\hline
\end{tabular}

$* p<0.05$. ${ }^{* *} p<0.01$.

호 
Table. 4 Correlations between Sexual Satisfaction and Relationship Characteristics

\begin{tabular}{|c|c|c|c|c|c|c|c|}
\hline Measure & 1 & 2 & 3 & 4 & 5 & 6 & 7 \\
\hline 1. Sexual Satisfaction & - & & & & & & \\
\hline 2. Number of partners ever married & .01 & - & & & & & \\
\hline 3. Number of people currently involved with & -.07 & $-0.28^{* *}$ & - & & & & \\
\hline 4. Number of times pregnant & -.10 & $0.27^{* *}$ & -0.11 & - & & & \\
\hline 5. Time involved with current partner - days & -.09 & -0.09 & $0.16^{*}$ & 0.09 & - & & \\
\hline 6. Time involved with current partner - months & $-.13^{*}$ & 0.00 & 0.08 & 0.01 & $0.28^{* *}$ & - & \\
\hline 7. Time involved with current partner - years & .05 & $0.42^{* *}$ & $-0.37^{* *}$ & $0.23^{* *}$ & $-0.26^{* *}$ & $-0.16^{* *}$ & - \\
\hline 8. Relationship type & $.33^{*}$ & -0.16 & 0.06 & 0.23 & 0.12 & -0.06 & -0.21 \\
\hline 9. Enjoy doing ordinary things with partner & $.46^{* *}$ & -0.11 & 0.09 & $0.12^{*}$ & 0.04 & -0.09 & -0.03 \\
\hline 10. Satisfied handling problems with partner & $.51^{* *}$ & -0.10 & -0.02 & 0.03 & 0.04 & -0.12 & -0.03 \\
\hline 11. Satisfied handling finances with partner & $.49^{* *}$ & $-0.12^{*}$ & 0.06 & -0.01 & 0.01 & -0.07 & -0.11 \\
\hline 12. Partner listens & $.52^{* *}$ & -0.05 & 0.01 & 0.11 & 0.01 & $-0.19^{* *}$ & 0.08 \\
\hline 13. Partner expresses love and affection & $.54^{* *}$ & $-0.12^{*}$ & 0.04 & 0.11 & 0.09 & -0.08 & 0.00 \\
\hline 14. Trusts partner to be faithful & $.46^{* *}$ & -0.10 & 0.11 & 0.07 & 0.05 & -0.06 & -0.03 \\
\hline 15. Extent of love for current partner & $.40^{* *}$ & $-0.15^{*}$ & $0.32^{* *}$ & -0.04 & 0.09 & -0.05 & $-0.19^{* *}$ \\
\hline 16. Extent of happiness with current partner & $.53^{* *}$ & -0.10 & $0.19^{* *}$ & 0.06 & 0.10 & -0.13 & -0.05 \\
\hline 17. Extent of commitment to current partner & $.47^{* *}$ & -0.12 & $0.32^{* *}$ & -0.09 & 0.09 & -0.08 & $-0.15^{*}$ \\
\hline 18. Frequency of sexual activity & $.31^{* *}$ & $-0.14^{*}$ & 0.05 & -0.06 & 0.04 & -0.06 & $-0.16^{* *}$ \\
\hline 19. Extent of contraceptive use with partner & .01 & -0.04 & 0.03 & -0.12 & -0.07 & 0.03 & 0.00 \\
\hline
\end{tabular}

${ }^{*} p<0.05$. ${ }^{* *} p<0.01$. 
Table 4

Continued

\begin{tabular}{|c|c|c|c|c|c|c|c|c|}
\hline Measure & 8 & 9 & 10 & 11 & 12 & 13 & 14 & 15 \\
\hline 8. Relationship type & - & & & & & & & \\
\hline 9. Enjoy doing ordinary things with partner & $0.44^{* *}$ & - & & & & & & \\
\hline 10. Satisfied handling problems with partner & 0.19 & $0.61^{* *}$ & - & & & & & \\
\hline 11. Satisfied handling finances with partner & $0.28^{*}$ & $0.52^{* *}$ & $0.65^{* *}$ & - & & & & \\
\hline 12. Partner listens & $0.41^{* *}$ & $0.66^{* *}$ & $0.66^{* *}$ & $0.55^{* *}$ & - & & & \\
\hline 13. Partner expresses love and affection & $0.45^{* *}$ & $0.67^{* *}$ & $0.63^{* *}$ & $0.49^{* *}$ & $0.66^{* *}$ & - & & \\
\hline 14. Trusts partner to be faithful & $0.33^{*}$ & $0.64^{* *}$ & $0.54^{* *}$ & $0.44^{* *}$ & $0.61^{* *}$ & $0.68^{* *}$ & - & \\
\hline 15. Extent of love for current partner & $0.63^{* *}$ & $0.54^{* *}$ & $0.37^{* *}$ & $0.31^{\text {** }}$ & $0.49^{* *}$ & $0.51^{* *}$ & $0.50^{* *}$ & - \\
\hline 16. Extent of happiness with current partner & $0.49^{* *}$ & $0.69^{* *}$ & $0.58^{* *}$ & $0.47^{* *}$ & $0.59^{* *}$ & $0.65^{* *}$ & $0.66^{* *}$ & $0.67^{* *}$ \\
\hline 17. Extent of commitment to current partner & $0.67^{* *}$ & $0.50^{* *}$ & $0.39^{* *}$ & $0.33^{* *}$ & $0.47^{* *}$ & $0.49^{* *}$ & $0.51^{* *}$ & $0.66^{* *}$ \\
\hline 18. Frequency of sexual activity & 0.23 & $0.27^{* *}$ & $0.20^{* *}$ & $0.18^{* *}$ & $0.22^{* *}$ & $0.20^{* *}$ & $0.22^{* *}$ & $0.27^{* *}$ \\
\hline 19. Extent of contraceptive use with partner & 0.00 & -0.08 & -0.05 & -0.03 & -0.08 & -0.08 & -0.07 & $-.13^{*}$ \\
\hline
\end{tabular}

${ }^{*} p<0.05$. ${ }^{* *} p<0.01$. 
Table 4

Continued

Measure 16

16

18

16. Extent of happiness with current partner

17. Extent of commitment to current partner $0.64^{* *}$

18. Frequency of sexual activity

$0.28^{* * *}$

19. Extent of contraceptive use with partner

$-0.07$

$0.18^{* *}$

$-0.12$

0.04

$* p<0.05 . * * p<0.01$. 
Table 5. One-way Analysis of Variance of Sexual Satisfaction in Latina Women by Subgroup

\begin{tabular}{lccccc}
\hline \multicolumn{1}{c}{ Source } & $d f$ & $S S$ & $M S$ & $F$ & $p$ \\
\hline Between Groups & 5 & 1.43 & 0.29 & 0.26 & 0.94 \\
Within Groups & 266 & 297.45 & 1.12 & & \\
Total & 271 & 298.88 & & & \\
\hline
\end{tabular}

Sexual Satisfaction Scores for Latina Women by Ethnicity

\begin{tabular}{lcccc}
\hline & $\mathrm{N}$ & $\mathrm{M}(\mathrm{SD})$ & \multicolumn{2}{c}{$95 \% \mathrm{CI}$} \\
\cline { 4 - 5 } Mexican & 143 & $1.83(1.08)$ & 1.65 & 2.00 \\
Puerto Rican & 34 & $1.85(1.10)$ & 1.47 & 2.24 \\
Central \& South American & 31 & $1.97(1.08)$ & 1.57 & 2.36 \\
Cuban & 11 & $1.64(0.67)$ & 1.18 & 2.09 \\
Chicana & 19 & $1.68(0.67)$ & 1.36 & 2.01 \\
Other & 34 & $1.82(1.17)$ & 1.42 & 2.23 \\
Total & 272 & $1.83(1.05)$ & 1.70 & 1.95 \\
\hline
\end{tabular}

Note: $C I=$ Confidence Interval; $L L=$ Lower Limit; $U L=$ Upper Limit 
Table 6. One-way Analysis of Variance of Number of Vaginal Sex Partners in Latina Women by Ethnicity

\begin{tabular}{lccccc}
\hline \multicolumn{1}{c}{ Source } & $d f$ & $S S$ & $M S$ & $F$ & $p$ \\
\hline Between Groups & 5 & 226.701 & .281 & .863 & .935 \\
Within Groups & 244 & 12816.98 & 1.088 & & \\
Total & 249 & 13043.68 & & & \\
\hline
\end{tabular}

Number of Sexual Partners for Latina Women by Ethnicity

\begin{tabular}{lcccc} 
& \multirow{2}{*}{$\mathrm{N}$} & $\mathrm{M}(\mathrm{SD})$ & \multicolumn{2}{c}{$95 \% \mathrm{CI}$} \\
\cline { 4 - 5 } Mexican & 131 & $4.99(6.97)$ & 4.99 & 7.40 \\
Puerto Rican & 29 & $7.31(5.34)$ & 5.28 & 9.34 \\
Central \& South American & 30 & $5.70(6.79)$ & 3.16 & 8.24 \\
Cuban & 9 & $2.67(1.66)$ & 1.39 & 3.94 \\
Chicana & 18 & $5.67(3.52)$ & 1.39 & 3.94 \\
Other & 33 & $5.67(11.36)$ & 3.92 & 7.41 \\
Total & 250 & $6.29(7.24)$ & 5.39 & 7.19 \\
\hline
\end{tabular}

Note: $C I=$ Confidence Interval; $L L=$ Lower Limit; $U L=$ Upper Limit 
Table 7. Regression of Sexual Satisfaction on Time Involved with Current Partner Controlling for Income, Education and Religion

\begin{tabular}{|c|c|c|c|c|c|c|}
\hline Source & & $B$ & $S E$ & $\beta$ & $t$ & $p$ \\
\hline $\begin{array}{l}\text { Time involved with } \\
\text { current partner }\end{array}$ & & -.03 & .03 & -.08 & -1.26 & .21 \\
\hline Total household income & & -.05 & .03 & -.12 & -1.71 & .09 \\
\hline Highest education level & & .05 & .03 & .11 & 1.57 & .12 \\
\hline Religion & & .04 & .04 & .06 & .85 & .40 \\
\hline Intercept & & 1.97 & .33 & & 5.93 & $<0.01 * *$ \\
\hline$d f$ & 230 & & & & & \\
\hline$F$ & 1.88 & & & & & \\
\hline$R$ & .18 & & & & & \\
\hline$R^{2}$ & .03 & & & & & \\
\hline
\end{tabular}

Table 8. Regression of Sexual Satisfaction on Relationship Satisfaction Controlling for Income, Education \& Religion

\begin{tabular}{|c|c|c|c|c|c|c|}
\hline Source & & $B$ & $S E$ & $\beta$ & $t$ & $p$ \\
\hline Relationship & & .80 & .07 & .64 & 12.43 & $<0.01 * *$ \\
\hline \multicolumn{7}{|l|}{ Satisfaction } \\
\hline Total household income & & .01 & .02 & .02 & .36 & .72 \\
\hline Highest education level & & .05 & .03 & .10 & 1.86 & .06 \\
\hline Religion & & .01 & .03 & .01 & .18 & .86 \\
\hline Intercept & & .04 & .29 & & .15 & .884 \\
\hline$d f$ & 242 & & & & & \\
\hline$F$ & $41.18 * *$ & & & & & \\
\hline$R$ & .64 & & & & & \\
\hline$R^{2}$ & .41 & & & & & \\
\hline
\end{tabular}

Table 9. Regression of Sexual Satisfaction on Number of Sexual Partners Controlling for Income, Education, and Religion

\begin{tabular}{|c|c|c|c|c|c|c|}
\hline Source & & $B$ & $S E$ & $\beta$ & $t$ & $p$ \\
\hline Number of Partners & & .02 & .01 & .15 & 2.30 & $0.02 *$ \\
\hline $\begin{array}{l}\text { Total household } \\
\text { income }\end{array}$ & & -.08 & .03 & -.18 & -2.55 & $0.01 *$ \\
\hline Highest education level & & .05 & .03 & .10 & 1.43 & .16 \\
\hline Present religion & & .01 & .05 & .01 & .13 & .90 \\
\hline Intercept & & 2.01 & .33 & & 6.05 & $<0.01 * *$ \\
\hline$d f$ & 227 & & & & & \\
\hline$F$ & $3.26^{* *}$ & & & & & \\
\hline$R$ & .24 & & & & & \\
\hline
\end{tabular}




\begin{tabular}{|c|c|c|c|c|c|c|}
\hline$R^{2}$ & .06 & & & & & \\
\hline $\begin{array}{l}* p<0.05 . * * p<0.01 \\
\text { Table 10. Regression of } \\
\text { Income, Education, and }\end{array}$ & $\begin{array}{l}\text { Sexual } \mathrm{S} \\
\text { Religion }\end{array}$ & ffactio & Freq & $y$ of $S$ & ontroll & \\
\hline Source & & $B$ & $S E$ & $\beta$ & $t$ & $p$ \\
\hline Frequency of sex & & .39 & .09 & .29 & 4.14 & $<0.01 * *$ \\
\hline $\begin{array}{l}\text { Total household } \\
\text { income }\end{array}$ & & -.02 & .03 & -.05 & -.75 & .46 \\
\hline Highest education level & & .03 & .03 & .05 & .81 & .42 \\
\hline Present religion & & .01 & .04 & .02 & .33 & .74 \\
\hline Intercept & & 1.20 & .34 & & 3.56 & $<0.01 * *$ \\
\hline$d f$ & 235 & & & & & \\
\hline$F$ & $6.23 * *$ & & & & & \\
\hline$R$ & .31 & & & & & \\
\hline$R^{2}$ & .10 & & & & & \\
\hline
\end{tabular}

Table 11. Regression of Sexual Satisfaction on Individual Relationship Variables on for all Latinas

\begin{tabular}{|c|c|c|c|c|c|c|}
\hline Source & & $B$ & $S E$ & $\beta$ & $t$ & $p$ \\
\hline $\begin{array}{l}\text { Enjoy ordinary things } \\
\text { together }\end{array}$ & & -.04 & .10 & -.04 & -.43 & .67 \\
\hline $\begin{array}{l}\text { Satisfied handling } \\
\text { problems w/partner }\end{array}$ & & .16 & .07 & .17 & 2.14 & $.03 *$ \\
\hline $\begin{array}{l}\text { Satisfied handling } \\
\text { finances w/partner }\end{array}$ & & .19 & .06 & .22 & 3.50 & $.001^{* *}$ \\
\hline $\begin{array}{l}\text { Partner listens when } \\
\text { needs to talk }\end{array}$ & & .07 & .08 & . 08 & .90 & .37 \\
\hline $\begin{array}{l}\text { Partner expresses love } \\
\text { and affection }\end{array}$ & & .23 & .09 & .22 & 2.73 & $.01 *$ \\
\hline $\begin{array}{l}\text { Trust partner to be } \\
\text { faithful }\end{array}$ & & -.15 & .06 & -.17 & -2.43 & $.02 *$ \\
\hline $\begin{array}{l}\text { Extent of love for } \\
\text { current partner }\end{array}$ & & .14 & .12 & .09 & 1.12 & .26 \\
\hline $\begin{array}{l}\text { Extent R's happiness } \\
\text { w/current partner }\end{array}$ & & .27 & .15 & .16 & 1.82 & .07 \\
\hline $\begin{array}{l}\text { Extent } R \text { committed to } \\
\text { current partner }\end{array}$ & & .18 & .09 & .15 & 2.04 & $.04 *$ \\
\hline Intercept & & .04 & .13 & & .31 & .76 \\
\hline $\begin{array}{l}d f \\
F\end{array}$ & $\begin{array}{c}239 \\
28.79 * *\end{array}$ & & & & & \\
\hline$R$ & .73 & & & & & \\
\hline
\end{tabular}


$\frac{R^{2}}{{ }^{*} p<0.05 .{ }^{* *} p<0.01 .}$

Table 12. Regression of Sexual Satisfaction on Number of Vaginal Sex Partners, Frequency of Sexual Activity and Time Involved with Current Partner for all Latinas

\begin{tabular}{|c|c|c|c|c|c|c|}
\hline Source & & $B$ & $S E$ & $\beta$ & $t$ & $p$ \\
\hline $\begin{array}{l}\text { Number of vaginal sex } \\
\text { partners }\end{array}$ & & .02 & .01 & .16 & 2.53 & $<0.01 * *$ \\
\hline $\begin{array}{l}\text { Frequency of sexual } \\
\text { activity }\end{array}$ & & .46 & .08 & .33 & 5.49 & $<.01^{* *}$ \\
\hline $\begin{array}{l}\text { Time involved } \\
\text { w/current partner }\end{array}$ & & .03 & .02 & .10 & 1.58 & .12 \\
\hline Intercept & & .88 & .19 & & 4.64 & $<0.01 * *$ \\
\hline $\begin{array}{l}d f \\
F\end{array}$ & $\begin{array}{c}240 \\
12.86^{* *}\end{array}$ & & & & & \\
\hline$R$ & .37 & & & & & \\
\hline$R^{2}$ & .14 & & & & & \\
\hline
\end{tabular}




\section{FIGURES}

Figure 1. Histogram for birth year for all Latinas.

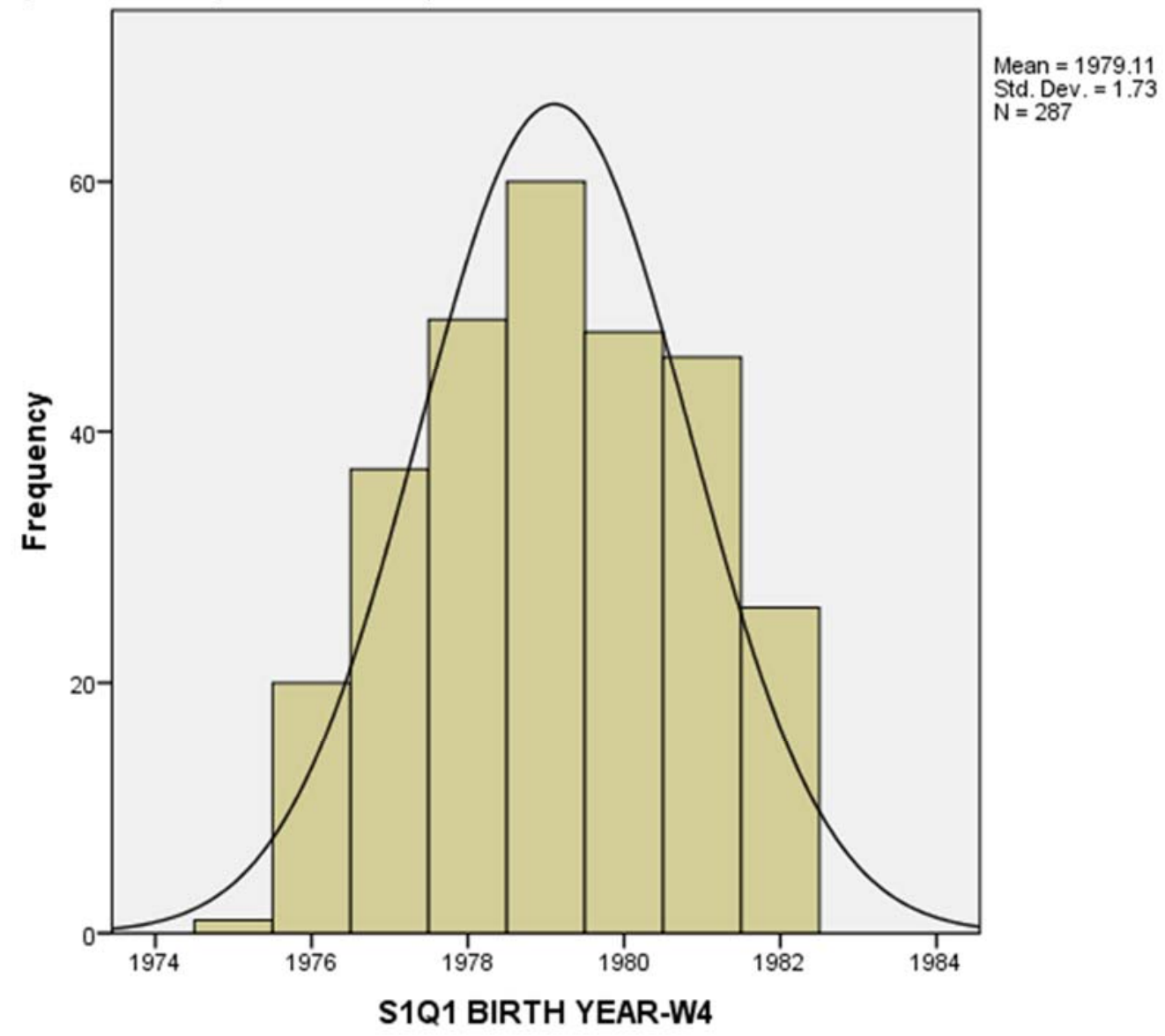


Figure 2. Histogram for household income for all Latinas.

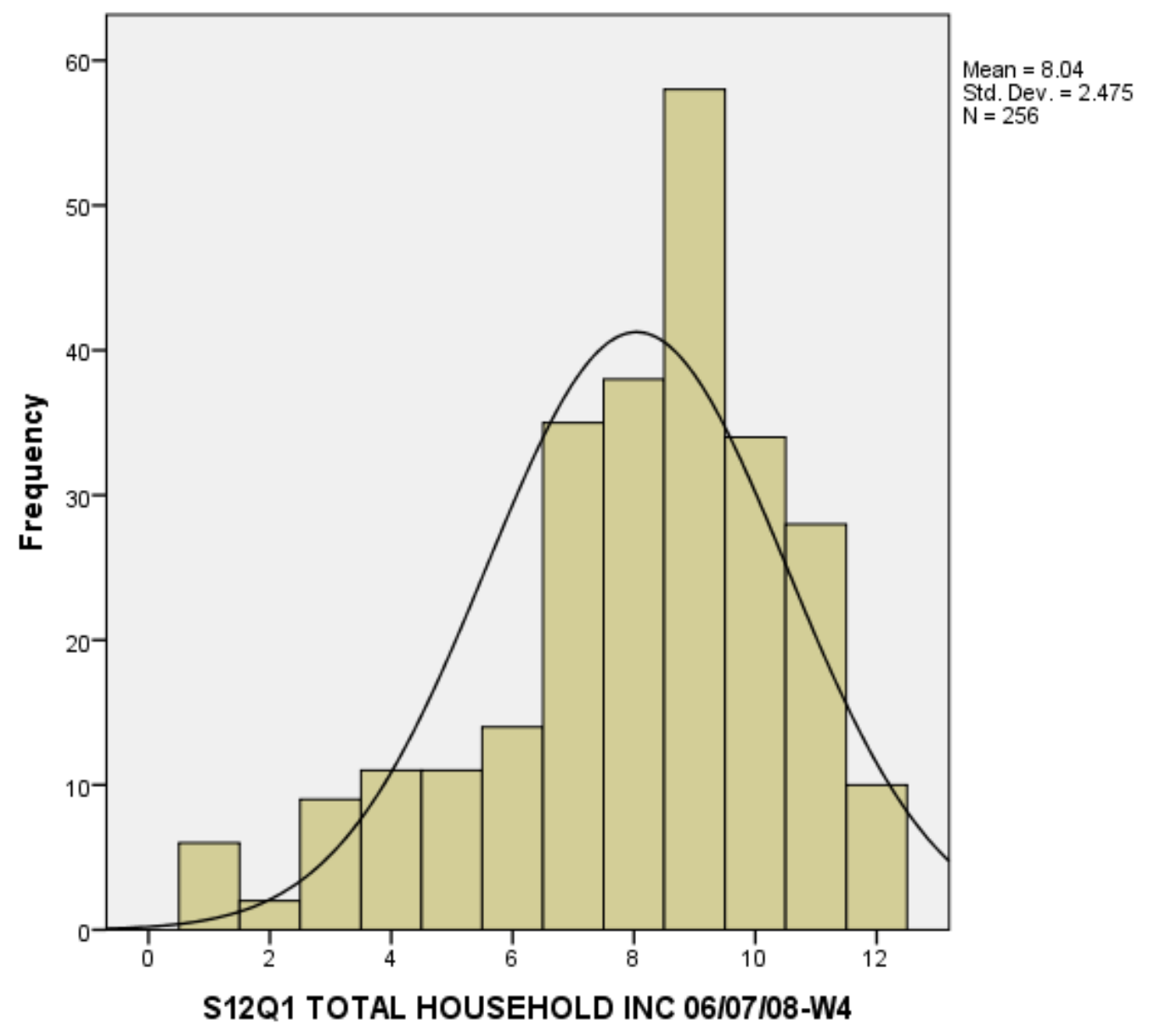


Figure 3. Histogram for number of vaginal partners ever had for all Latina group.

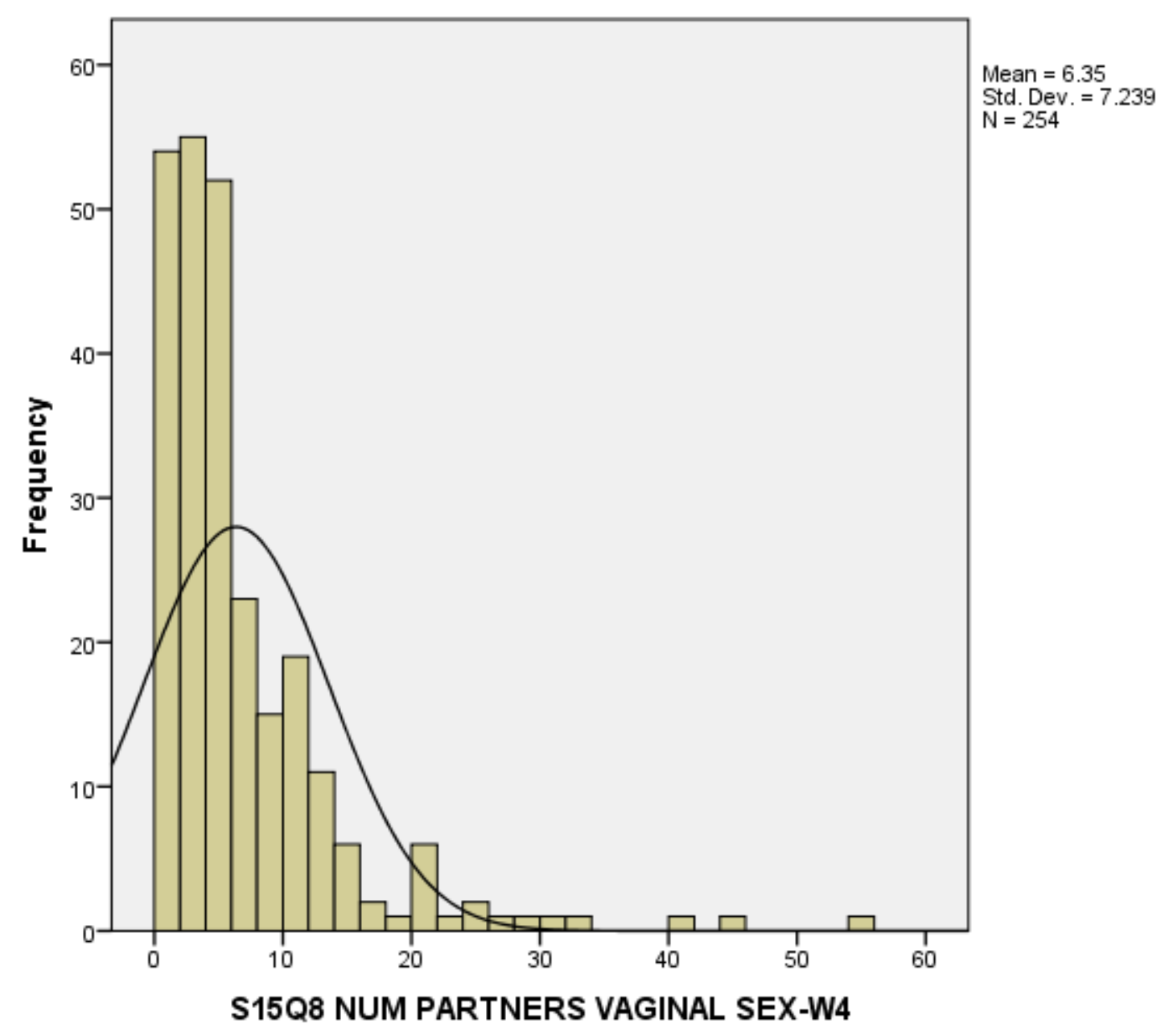


Figure 4. Pie chart for Education for Latinas.

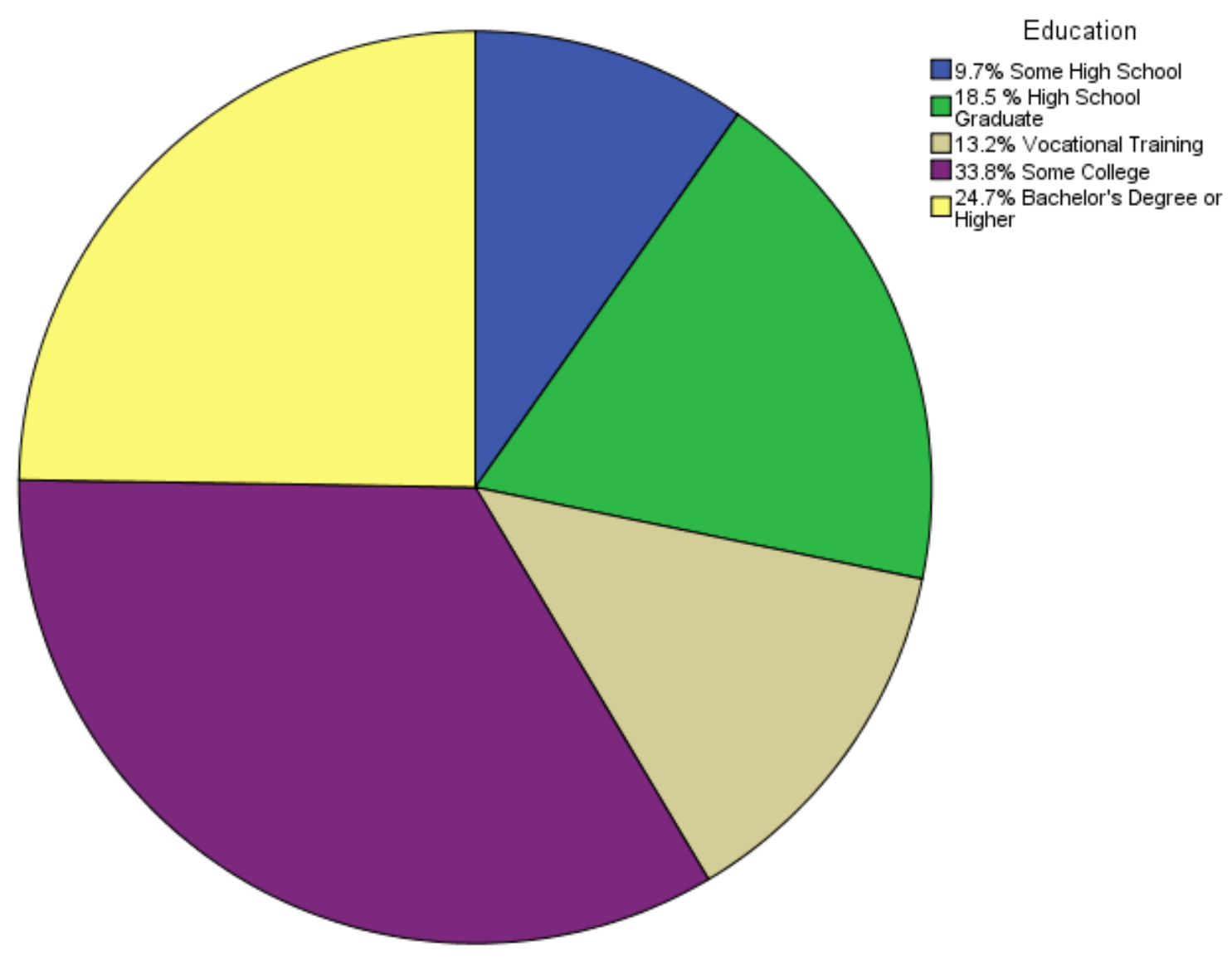


Figure 5. Means plot of sexual satisfaction and Latina subgroups.

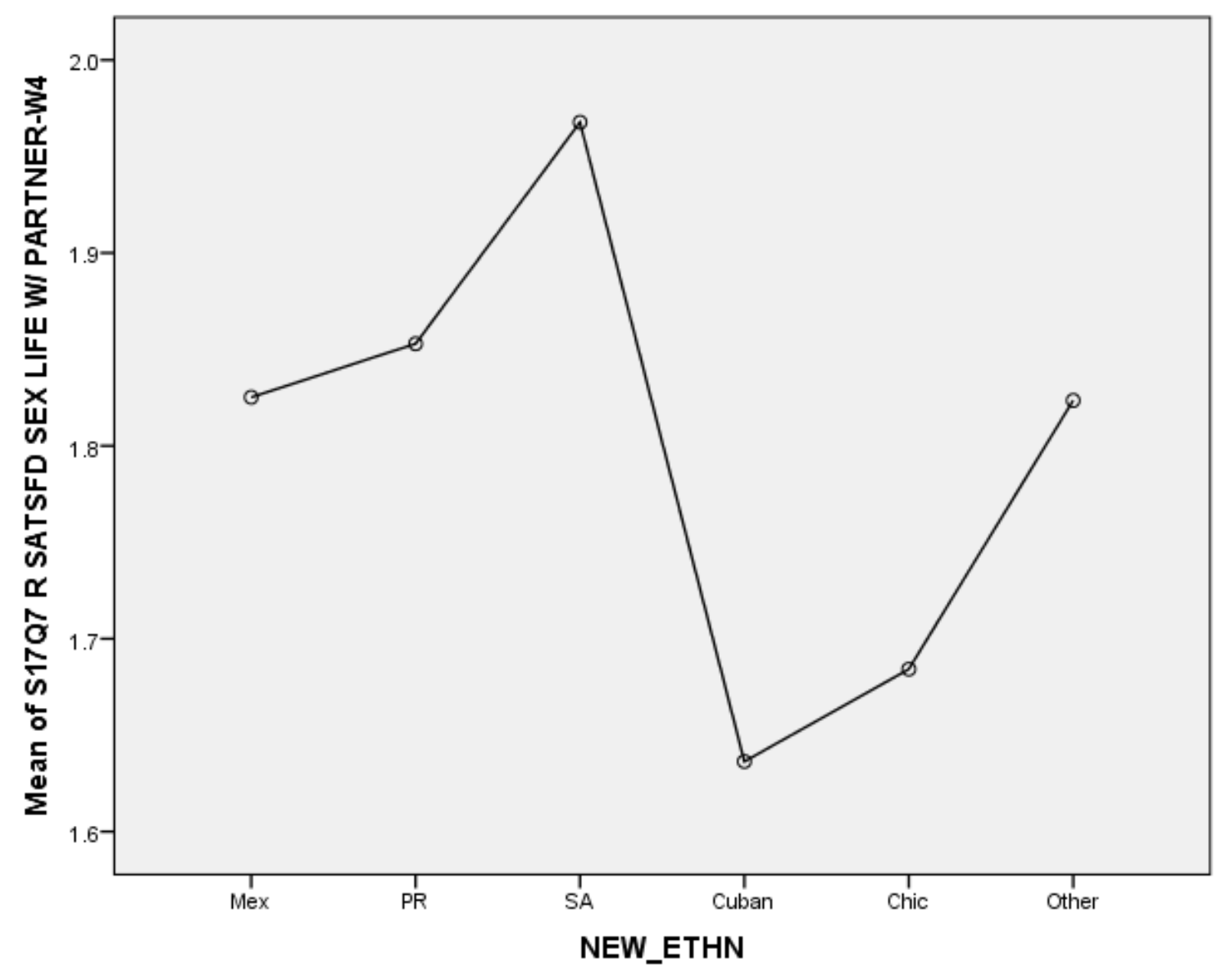


Figure 6. Means plot of number of vaginal sex partners among Latina subgroups.

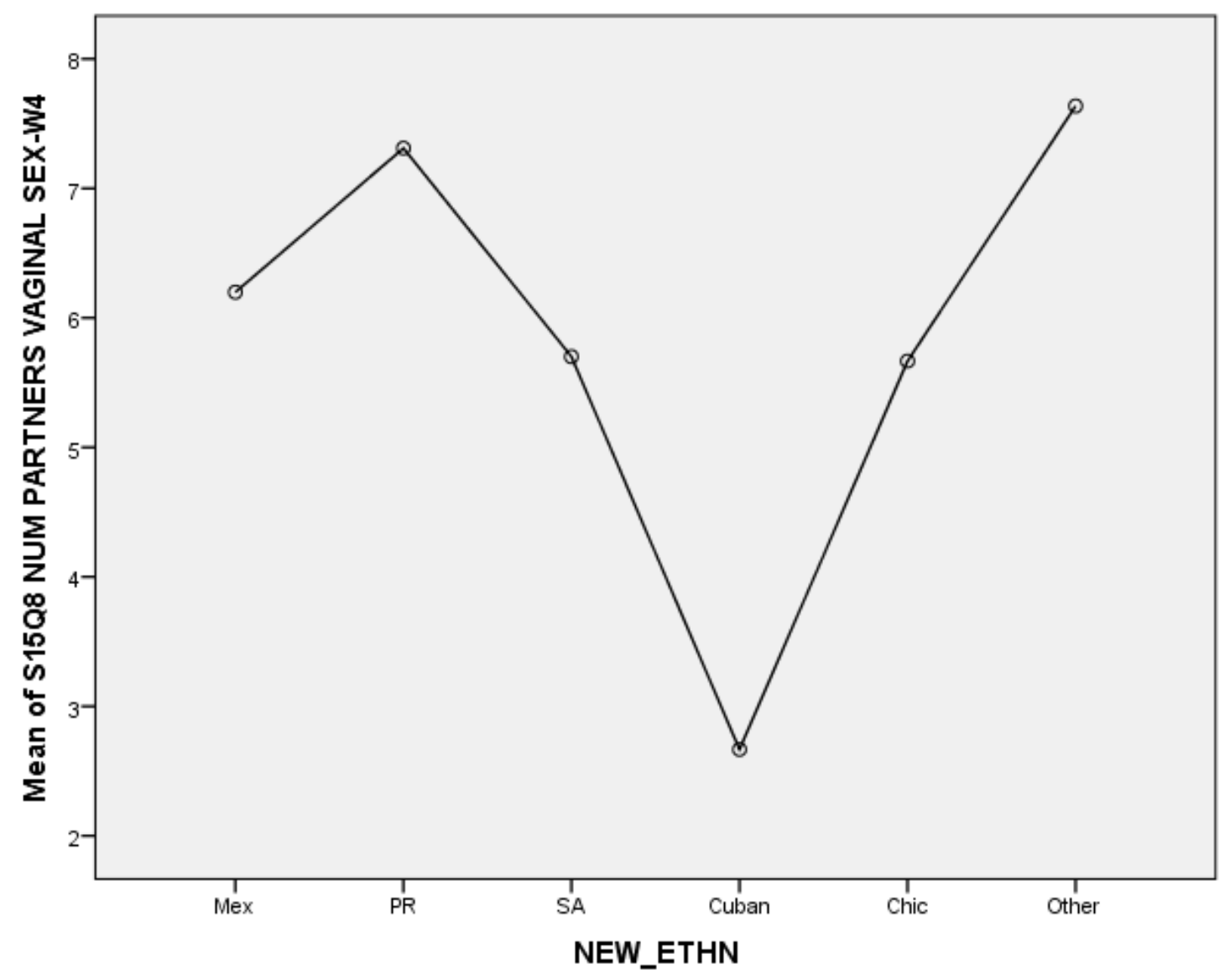


Figure 7. Stem and Leaf Plot for Number of Vaginal Sex Partners for Puerto Rican Latina

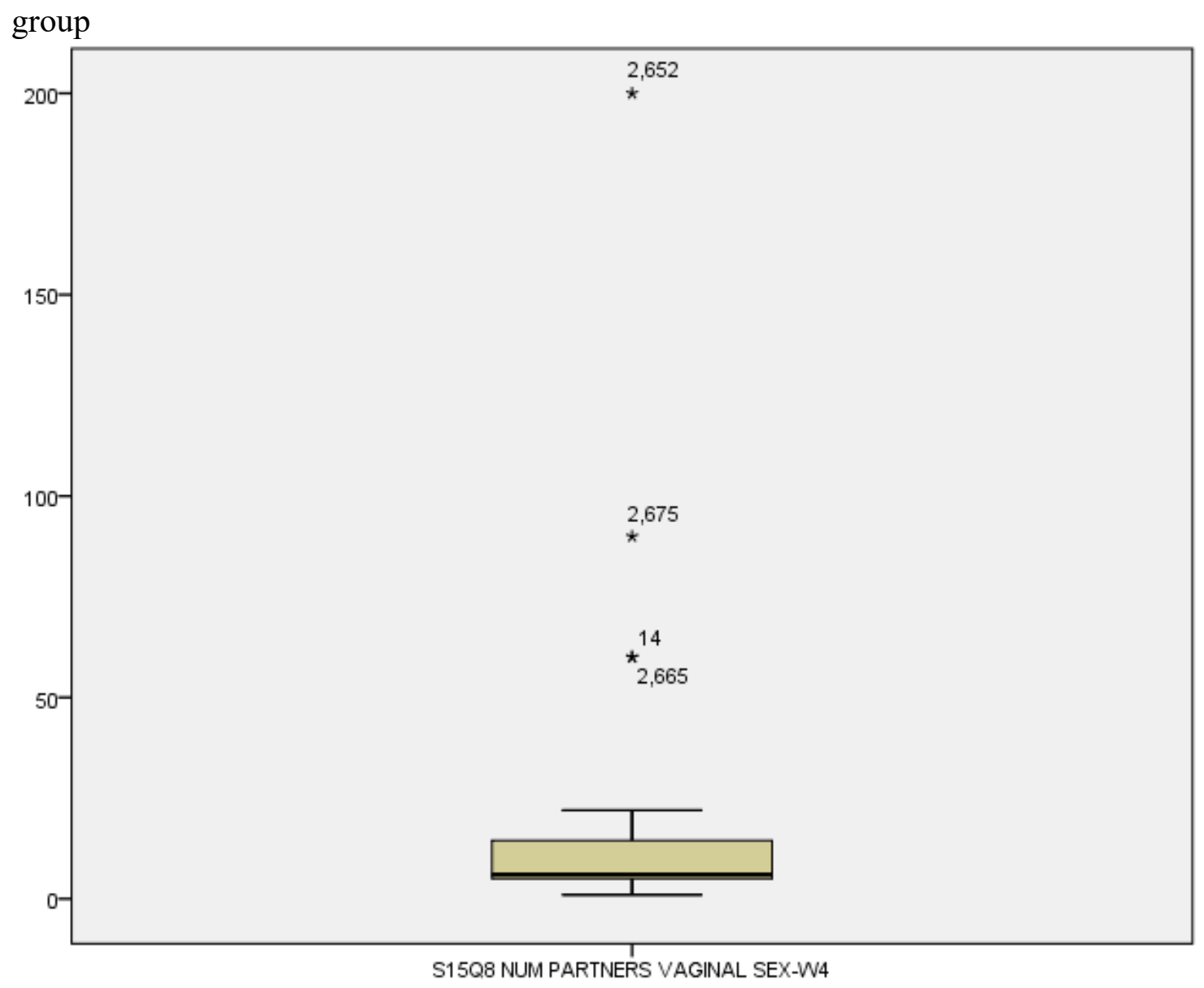




\section{REFERENCES}

Barrientos, J.E. \& Páez, D. (2006). Psychosocial variables of sexual satisfaction in Chile. Journal of Sex and Marital Therapy, 32(5), 351-368. DOI: $10.1080 / 00926230600834695$

Adler, N. E., Cutler, D. M., Jonathan, J. E., Galea, S., Glymour, M., Koh, H. K., \& Satcher, D. (2016). Addressing social determinants of health and health disparities. Discussion Paper, Vital Directions for Health and Health Care Series. National Academy of Medicine, Washington, DC. https://nam. edu/wpcontent/uploads/2016/09/addressing-social-determinantsof-health-and-healthdisparities. pdf.

Afable-Munsuz, A. \& Brindis, C.D. (2006). Acculturation and the sexual and reproductive health of Latino youth in the United States: a literature review. Perspectives on Sexual and Reproductive Health, 38(4), 208-219.

Alamilla, S.G., Kim, B.S.K., \& Lam, N.A. (2010). Acculturation, enculturation, perceived racism, minority status stressors, and psychological symptomology among Latinos/as. Hispanic Journal of Behavioral Sciences, 32, 55-76.

Alcoff, L. M. (2005). Latino vs. Hispanic: The politics of ethnic names. Philosophy \& Social Criticism, 31(4), 395-407.

Alvarez, C.P. \& Villarruel, A. (2013). Sexual communication among young adult heterosexual Latinos: a qualitative descriptive study. Hispanic Health Care International, 11(3), 101-110.

Alvarez, C. \& Villarruel, A. (2015). Association of gender norms, relationship and intrapersonal variables, and acculturation with sexual communication among young adult Latinos. Research in Nursing and Health, 38, 121-132.

Alzate, M.M. (2009). The role sexual and reproductive rights in social work practice. Affilia: Journal of Women and Social Work, 24(2), 108-119.

Anzaldúa, G. (1987). Borderlands, la frontera: the new mestiza. San Francisco: Aunt Lute Books.

Arevalo, S. \& Amaro, H. (2010). Sexual health of Latina/o populations in the United States. In Asencio, M. (Ed), Latina/o Sexualities (pp.75-89). New Brunswick, NJ: Rutgers University Press. 
Asencio, M. \& Acosta, K. (2010). Mapping Latina/o sexualities research and scholarship. In Asencio, M. (Ed), Latina/oSexualities (pp. 1-12). New Brunswick, NJ: Rutgers University Press.

Arrizón, A. (2008). Latina subjectivity, sexuality and sensuality. Women \& Performance: A Journal of Feminist Theory, 18(3), 189-198.

Bernal, D. D. (2002). Critical race theory, Latino critical theory, and critical racedgendered epistemologies: Recognizing students of color as holders and creators of knowledge. Qualitative inquiry, 8(1), 105-126.

Bauer, G.R. (2014). Incorporating intersectionality theory into population health research methodology: challenges and the potential to advance health equity. Social Science and Medicine, 110, 10-17.

Baquero, B., Goldman, S.N., Siman, F., Muqueeth, S., Eng, E., Rhodes, S.D. (2011). Mi cuerpo, nuestro responsabilidad: using photovoice to describe the assets and barriers to sexual and reproductive health among Latinos. Journal of Health Disparities Research and Practice, 7(1), 65-83.

Barrientos, J., \& Páez, D. (2006). Psychosocial Variables of Sexual Satisfaction in Chile. Journal of Sex \& Marital Therapy., 32(5), 351-368.

Bay-Cheng, L., Livingston, J.A., \& Fava, N.M. (2010). Adolescent girls' assessment and management of sexual risks: insights from focus group research. Youth and Society, 1-27. DOI: 10.1177/004418X10384475

Bay-Cheng, L.Y. (2013). Human sexuality. In T. Mizrahi \& L.E. Davis (Eds-in-chief), Encyclopedia of social work (online ed.). Washington, DC and New York:

NASW Press and Oxford University Press. doi: 10.1093/acrefore/9780199975839.013.191

Bowleg, L. (2008). When black + lesbian + woman $\neq$ black lesbian woman: the methodological challenges of qualitative and quantitative intersectionality research. Sex Roles, 59, 312-325.

Bowleg, L. (2012). The problem with the phrase women and minorities: intersectionalityan important theoretical framework for public health. Framing Health Matters, 102(7), 1267-1273.

Braveman, P., \& Gottlieb, L. (2014). The social determinants of health: it's time to consider the causes of the causes. Public Health Reports, 129(1_supp12), 19-31.

Brown, A. (2014). US Hispanic and Asian populations growing, but for different reasons. Pew Research Center, retrieved from http://www.pewresearch.org/fact- 


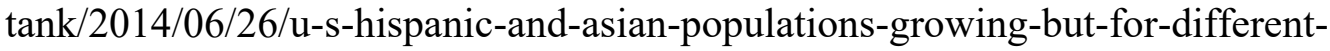
reasons/

Buhi, E.R., Marehefka, S.L. \& Hoban, M.T. (2010). The state of the union: sexual health disparities in a national sample of US college students. Journal of American College Health, 58(4), 337-346.

Buhi, E. R., Marhefka, S. L., Wheldon, C. W., Tilley, D. L., Klinkenberger, N., Lescano, C., \& Hoban, M. T. (2014). Sexual and reproductive health disparities in a national sample of Hispanic and non-Hispanic White US college students. Journal of Health Disparities Research and Practice, 7(1), 3.

Buikema, R., Griffin, G. \& Lykke, N. (2011). Theories and Methodologies in Postgraduate Feminist Research. New York: Routledge.

Carvajal, D.N., Gioia, D., Mudafort, E.R., Brown, P.B., \& Barnet, B. (2017). How can primary care physicians best support contraceptive decision making? A qualitative study exploring the perspectives of Baltimore Latinas. Women's Health Issues, 27(2), 158-166.

Cashman, R., Eng, E., Simán, F. \& Rhodes, S.D. (2011). Exploring the sexual health priorities and needs of immigrant Latinas in the southeastern United States: a community based participatory approach. AIDS Education and Prevention, 23(3), 236-248.

Castañeda, D.M. (2017). Sexting and sexuality in romantic relationships among Latina/o emerging adults. American Journal of Sexuality Education, 12(2), 120-135.

Castro-Salazar, R. \& Bagley, C. (2012). Navigating Borders. New York: Peter Lang Publishing.

Champeau, D. A., \& Shaw, S. M. (2003). Teaching about interlocking oppressions: The case of HIV and women. Feminist Teacher, 208-219.

Cheng, S., Hamilton, L., Missari, S. \& Ma, J. (2014). Sexual subjectivity among adolescent girls: social disadvantage and young adult outcomes. Social Forces, 93(2), 515-544. DOI: 10.1093/sf/sou084

Centers for Disease Control (CDC) (2016). Retrieved from https://www.cdc.gov/nchhstp/healthdisparities/hispanics.html

Centers for Disease Control (CDC) (2016) Retrieved from https://www.cdc.gov/nchhstp/newsroom/docs/factsheets/cdc-hiv-latinos-508.pdf 
Cole, E.R. (2009). Intersectionality and research in psychology. American Psychologist, 64(3), 170-180.

Collins, P.H. \& Bilge, S. (2016). Intersectionality. Malden, MA: Polity Press.

Collins, P.H. (1991). Black Feminist Thought: Knowledge, Consciousness, and the 20 Politics of Empowerment. New York, NY: Routeldge.

Cowan, B. A. (2017). How Machismo Got Its Spurs—in English: Social Science, Cold War Imperialism, and the Ethnicization of Hypermasculinity. Latin American Research Review, 52(4).

Crenshaw, K. (1993). Mapping the margins: Intersectionality identity politics, and violence against women of color. Stanford Law Review, 43, 1241-1299.

Crenshaw, K.W. (1995). The intersection of race and gender. In K.W.C, N.G., G.P., K.T. (Eds). Critical Race Theory: The Key Writings that Informed the Movement (pp.357-383). New York, NY: The New Press.

Cronk, B. (2016). How to use SPSS. Glendale, CA: Pyrczak Publishing.

Deardorff, J., Tschann, J.M., Flores, E., \& Ozer, E.J. (2010). Sexual values and risky behaviors among Latino youths. Perspectives on Sexual and Reproductive Health, $42,23-32$.

Delgado, R. \& Stefancic, J. (2000). Latino/a critical (“LatCrit”) legal studies: review essay. Aztlán: A Journal of Chicano Studies, 25(2), 161-189.

Delgado, R. \& Stefancic, J. (2001). Critical race theory: an introduction. New York, NY: New York University Press.

DeLamater, J., \& Hyde, J. (1998). Essentialism vs. Social Constructionism in the Study of Human Sexuality. The Journal of Sex Research, 35(1), 10-18. Retrieved from http://www.jstor.org/stable/3813161

D’Orazio, L.M., Taylor-Ford, M. \& Meyerowitz, B.E. (2014). Cervical cancer prevention among Latinas in a post-HPV vaccine world: considering the sociocultural context. Women \& Therapy, 37(3-4), 264-281.

Dodd, S.J. \& Tolman, D. (2017). Reviving a positive discourse on sexuality within social work. Social Work, 62(3), 227-234.

Downing, R.A., LaVeist, T.A., \& Bullock, H. (2007). Intersections of ethnicity and social class in provider advice regarding reproductive health. American Journal of Public Health, 97(10), 1803-1807. 
Elze, D.E. (2003). Gay, lesbian, and bisexual youths' perceptions of their high school environments and comfort in school. Children \& Schools, 25, 225-239.

Epstein, S., \& Mamo, L. (2017). The proliferation of sexual health: Diverse social problems and the legitimation of sexuality. Social Science \& Medicine, 188, 176190.

Erel, U., Haritaworn, J., Rodriguez, E.G., \& Klesse, C. (2011). On the depoliticization of intersectionality talk: conceptualizing multiple oppressions in critical sexuality studies. In Taylor, Y., Hines, S. \& Casey, M.E. (Eds.), Theorizing Intersectionality and Sexuality (pp.56-77). Great Britain: Palgrave Macmillan.

Fahs, B. \& Swank, E. (2011). Social identities as predictors of women's sexual satisfaction and sexual activity. Archives of Sexual Behavior, 40, 903-914.

Farmer, M.A. \& Meston, C.M. (2006). Predictors of condom use self-efficacy in an ethnically diverse university sample. Archives of Sexual Behavior, 35(3), 313326.

Faulkner, S. L. (2003). Good girl or flirt girl: Latinas' definitions of sex and sexual relationships. Hispanic Journal of Behavioral Sciences, 25(2), 174-200.

Field, A. (2013). Discovering Statistics Using SPSS, IBM SPSS Statistics $\left(4^{\text {th }}\right.$ Ed). Thousand Oaks, CA: Sage Publications.

Fine, M. \& McClelland, S.I. (2006). Sexuality education and desire: still missing after all these years. Harvard Educational Review, 76(3), 297-338.

Finch, B.K., Do, D.P., Frank, R.F., \& Seeman, T. (2009). Could "acculturation" effects be explained by latent health disadvantages among Mexican immigrants? The International Migration Review, (43)3, 471-495.

Ford, C.L. and Airhihenbuwa, C.O. (2010a). The public health critical race methodology: praxis for antiracism research. Social Science and Medicine, 71, 1390-1398.

Ford, C.L. and Airhihenbuwa, C.O. (2010b). Critical race theory, race equity, and public health: toward antiracism praxis. American Journal of Public Health, 1(100), 3035 .

Ford, J. V., Ivankovich, M. B., Douglas Jr, J. M., Hook III, E. W., Barclay, L., Elders, J., ... \& Coleman, E. (2017). The Need to Promote Sexual Health in America: A New Vision for Public Health Action. Sexually transmitted diseases, 44(10), 579-585. 
Gandara, P. \& The White House Initiative on Educational Excellence for Hispanics (WHIDEH) (2015). Retrieved from https://sites.ed.gov/hispanicinitiative/files/2015/09/Fulfilling-Americas-Future-Latinas-in-the-U.S.-2015Final-Report.pdf

Garcia, L. (2009). Heteronormativity, sexism, and racism in the sexual (mis)education of Latina youth. Gender \& Society, 23(4), 520-541.

Garcia, L. (2012). Respect yourself, protect yourself: Latina girls and sexual identity. New York, NY: New York University Press.

Garcés-Palacio, M., Altarac, M. \& Scarinci, I.C. (2008). Contraceptive knowledge and use among low-income Hispanic immigrant women and non-Hispanic women. Contraception, 77(4), 270-275.

Giami, A. (2003). Sexual health: the emergence, development, and diversity of a concept. Sexual Health, 1-35.

Gilliam, M.L., Warden, M., Goldstein, C. \& Tapia, B. (2004). Concerns about contraceptive side effects among young Latinas: a focus group approach. Contraception, 70(4), 299-305.

Giunta, N. \& Rowan, N. (2015). Lesbian, gay, bisexual, and transgender aging: the role of gerontological social work. London/New York: Routeledge.

Gloria, A. M., \& Castellanos, J. (2013). Realidades culturales y identidades dimensionadas: The complexities of Latinas' diversities. The Oxford handbook of feminist multicultural counseling psychology, 169-182.

González-López, G., \& Vidal-Ortiz, S. (2008). Latinas and Latinos, sexuality, and society: A critical sociological perspective. Latinas/os in the United States: Changing the face of America, 308-322.

Gonzalez, E. U., Sable, M. R., Campbell, J. D., \& Dannerbeck, A. (2010). The influence of patriarchal behavior on birth control access and use among recent Hispanic immigrants. Journal of immigrant and minority health, 12(4), 551-558.

Council on Social Work Education (CSWE) (2015). Educational policy and accreditation standards. Retrieved from https://www.cswe.org/getattachment/Accreditation/Accreditation-Process/2015EPAS/2015EPAS_Web_FINAL.pdf.aspx

Graham, L., Brown-Jeffy, S. Aronson, R. \& Stephens, C. (2011). Critical race theory as theoretical framework and analysis tool for population health research. Critical Public Health, 21(1), 81-93. 
Guarini, T. E., Marks, A. K., Patton, F., \& Coll, C. G. (2011). The immigrant paradox in sexual risk behavior among Latino adolescents: Impact of immigrant generation and gender. Applied Developmental Science, 15(4), 201-209.

Guerra-Reyes, L., Rush, B., Herbenick, D., Dodge, B., Reece, M., Schick, V., Sanders, S.A., \& Fortenberry, J.D. (2017). Sexual health and language dominance among Hispanic/Latino women and men: analysis of a nationally representative sample. Journal of Immigrant and Minority Health, 19, 1059-1072.

Gutierrez, R.A. (2010). A history of Latina/o sexualities. In Asencio, M. (Ed), Latina/o Sexualities (pp.13-37). New Brunswick, NJ: Rutgers University Press.

Gurr, B. (2011). Complex intersections: reproductive justice and Native American women. Sociology Compass, 5(8), 721-735.

Haavio-Mannila, E., \& Kontula, O. (1997). Correlates of increased sexual satisfaction. Archives of sexual behavior, 26(4), 399-419.

Hancock, A.M. (2007). When multiplication doesn't equal quick addition: examining intersectionality as a research paradigm. Perspectives on Politics, 5(1), 63-79.

Hancock, A.M. (2016). Intersectionality. New York, NY: Oxford University Press.

Hankivsky, O. \& Christoffersen, A. (2008). Intersectionality and the determinants of health: a Canadian perspective. Critical Public Health, 18(3), 271-283.

Harris, K.M. \& Udry, R.J. (2015). "National Longitudinal Study of Adolescent to Adult Health (Add Health) Wave IV 2008, UNC. DOI: 10.15139/S3/11920

Harvey, S.M., Henderson, J.T. \& Casillas, A. (2006). Factors associated with effective contraceptive use among a sample of Latina women. Women and Health, 43(2), $1-16$.

Heckert, C. (2017). Blaming machismo: How the social imaginary is failing men with HIV in Santa Cruz, Bolivia. Medical anthropology, 36(2), 157-169.

Henderson, A.W., Lehavot, K. \& Simoni, J.M.(2009). Ecological models of sexual satisfaction among lesbian/bisexual and heterosexual women. Archives of Sexual Behavior, 38, 50-65.

Hensel, D.J. \& Fortenberry, J.D. (2013). A multidimensional model of sexual health and sexual and prevention behavior among adolescent women. Journal pf Adolescent Health, 52(2), 219-227. 
Herbenick, D., Reece, M., Sanders, S., Dodge, B., Ghassemi, A., \& Fortenberry, J. D. (2009). Prevalence and characteristics of vibrator use by women in the United States: Results from a nationally representative study. The Journal of Sexual Medicine, 6(7), 1857-1866.

Hernandez, J. (2009). " Miss, You Look Like a Bratz Doll": On Chonga Girls and Sexual-Aesthetic Excess. NWSA Journal, 21(3), 63-90.

Higgins, J.A. (2014). Celebration meets caution: long acting reversible contraception (LARC)'s boons, potential busts, and the benefits of a reproductive justice approach. Contraception, 89(4), 237-241.

Higgins, J. A., \& Hirsch, J. S. (2007). The pleasure deficit: revisiting the "sexuality connection" in reproductive health. Perspectives on sexual and reproductive health, 39(4), 240-247.

Higgins, J., Hoffman, S., Graham, C., \& Sanders, S. (2008). Relationships between condoms, hormonal methods, and sexual pleasure and satisfaction: An exploratory analysis from the Women's Well-Being and Sexuality Study. Sexual Health, 5(4), 321.

Higgins, J. A., Mullinax, M., Trussell, J., Davidson Sr, J. K., \& Moore, N. B. (2011). Sexual satisfaction and sexual health among university students in the United States. American Journal of Public Health, 101(9), 1643-1654.

Hogarth, H., \& Ingham, R. (2009). Masturbation among young women and associations with sexual health: An exploratory study. Journal of Sex Research, 46(6), 558567.

hooks, B. (2009). Writing beyond race: living theory and practice. New York, NY: Routelegde.

Hulko, W. (2009). The time to context-contingent nature of intersectionality and interlocking oppressions. Affilia, 24, 44-57.

Hurtado, A. (1996). On a reflexive feminist theory of gender subordination. The Color of Privilege: Three Blasphemies on Race and Feminism (pp.123-160). Ann Arbor, MI: -University of Michigan Press

Hurtado, A. (2001). Sitios y lenguas: Chicanas theorize feminisms. Hypatia, 13, 134-161.

Hurtado, A. (2003). Voicing Chicana Feminisms. New York: New York University Press.

Hurtado, A. \& Sinha, M. (2016). Beyond Machísmo: Intersectional Latino Masculinities. Austin, TX: University of Texas Press. 
Jackson, A. V., Karasek, D., Dehlendorf, C., \& Foster, D. G. (2016). Racial and ethnic differences in women's preferences for features of contraceptive methods. Contraception, 93(5), 406-411.

Jackson, M. (2013). Center for American Progress, Fact Sheet: The State of Latinas in the United States retrieved from https://www.americanprogress.org/issues/race/reports/2013/11/07/79167/factsheet-the-state-of-latinas-in-the-united-states/

Jayaratne, T.E. \& Stewart, A.J. (2008). Quantitative and qualitative methods in the social sciences. In A.M. Jaggar (Ed.), Just methods: an interdisciplinary feminist reader (pp.44-57). Boulder, CO: Paradigm Publishers.

Kiehme, E. (2016). Latino critical perspective in social work. Social Work, 61(2), 119126.

Knofczynski, G.T. \& Mundfrom, D. (2008). Sample sizes when using multiple linear regression for prediction. Educational and Psychological Measurement, 68(3), 431-442.

Krumer-Nevo, M., Berkovitz-Romano, A., \& Komem, M. (2015). The study of girls in social work: Major discourses and feminist ideas. Journal of Social Work, 15(4), 425-446.

Koniak-Griffin, D., Brecht, M. L., Takayanagi, S., Villegas, J., Melendrez, M., \& Balcázar, H. (2015). A community health worker-led lifestyle behavior intervention for Latina (Hispanic) women: Feasibility and outcomes of a randomized controlled trial. International journal of nursing studies, 52(1), 75-87.

Krieger, N. \& Gruskin, S. (2001). Frameworks matter: ecosocial and health and human rights perspectives on disparities in women's health- the case of tuberculosis. Journal of the American Women's Association, 56(4), 137-142.

Krogstad, J.M. (2016). Key facts about how the Hispanic population is changing from the Pew Research Center retrieved 7/5/16 from http://www.pewresearch.org/facttank/2016/09/08/key-facts-about-how-the-u-s-hispanic-population-is-changing/

Laganà, L., \& Maciel, M. (2010). Sexual desire among Mexican-American older women: A qualitative study. Culture, Health \& Sexuality., 12(6), 705-719.

Leotti, S. M., \& Muthanna, J. S. (2015). Troubling the binary: A critical look at the dualistic construction of quantitative/qualitative methods in feminist social work research. Feminisms in Social Work Research, 170-186. 
Livingston, G. (2009). Hispanics, Health Insurance, and Health Care Access. Pew Research Center retrieved from http://www.pewhispanic.org/2009/09/25/hispanics-health-insurance-and-healthcare-access/

Macartney, S., Bishaw, A. \& Fontenot, K. (2013). Poverty Rates for Selected Detailed Race and Hispanic Groups by State and Place: 2007-2011 from the US Census Bureau. Retrieved July 22, 2017 from https://www.census.gov/prod/2013pubs/acsbr11-17.pdf

Mann, E.S. (2016). Latina girls, sexual agency, and the contradictions of neoliberalism. Sexuality Research and Social Policy, 1-11.

Martinez, O., Wu, E., Moya, E.M., Chavez-Baray, S., Dodge, B., Shultz, A.Z., \& LopezRios, J. (2014). Overcoming issues and challenges in serving the sexual health needs of Latino immigrant in the United States. The Health Education Monograph Series, 31(2), 43-51.

Masinter, L.M., Feinglass, J., \& Simon, M.A. (2013). Pregnancy intention and use of contraception among Hispanic women in the United States: data from the national survey of family growth, 2006-2010. Journal of Women's Health, 22(10), 862870.

McCave, E., Shepard, B. \& Winter, V.R. (2014). Human sexuality as a critical subfield in social work. Advances in Social Work, 15(2), 409-427.

McWilliams, C. (2011). “The Mexican problem.” In R.D. \& J.S. (Eds). The Latino Condition (pp. 149-150). New York: New York University Press.

Mehrotra, G. (2010). Toward a continuum of intersectionality theorizing for feminist social work scholarship. Affilia, 25(4), 471-430.

Morales-Aleman, M.M. \& Scarinci, I.C. (2016). Correlates and predictors of sexual health among adolescent Latinas in the United States: a systematic review of the literature, 2004-2005. Preventive Medicine, 87, 183-193.

Motel, S. \& Patten, C. (2013). Statistical Portrait of Hispanics in the United States, 2011. Retrieved from http://www.pewhispanic.org/2013/02/15/statistical-portrait-ofhispanics-in-the-united-states-2011/

Moreno, C.L. (2007). The relationship between culture, gender, structural factors, abuse, trauma, and HIV/AIDS for Latinas. Qualitative Health Research, 17, 340-352.

Neff, J. A. (2001). A confirmatory factor analysis of a measure of "machismo" among Anglo, African American, and Mexican American male drinkers. Hispanic Journal of Behavioral Sciences, 23(2), 171-188. 
Nylund, D. (2006). Critical multiculturalism, whiteness, and social work: Towards a more radical view of cultural competence. Journal of Progressive Human Services, 17(2), 27-42.

Oboler, S. (2011). Hispanics? That's what they call us. In R.D. \& J.S. (Eds). The Latino Condition (pp.8-10). New York: New York University Press.

Oriel, J. (2005). Sexual pleasure as a human right: Harmful or helpful to women in the context of HIV/AIDS?. In Women's Studies International Forum (Vol. 28, No. 5, pp. 392-404). Pergamon.

Park, Y. (2008). Facilitating Injustice: Tracing the Role of Social Workers in the World War II Internment of Japanese Americans. Social Service Review, 82(3), 447483.

Park, Y., \& Kemp, S. P. (2006). "Little alien colonies": Representations of immigrants and their neighborhoods in social work discourse, 1875-1924. Social Service Review, 80(4), 705-734.

Patten, E. (2016). Racial, gender wage gaps persist in US despite some progress. Pew Research Center retrieved from http://www.pewresearch.org/facttank/2016/07/01/racial-gender-wage-gaps-persist-in-u-s-despite-some-progress/

Pew Research Center (2017). Hispanic Trends retrieved from http://www.pewhispanic.org/

Pinkerton, S., Cecil, H., Bogart, L., \& Abramson, P. (2003). The pleasures of sex: An empirical investigation. Cognition \& Emotion, 17(2), 341-353.

Planned Parenthood (2017). Addressing Sexual and Reproductive Health Disparities among Latinos retrieved from https://www.plannedparenthood.org/files/2814/2773/6927/Latino_Disparities.pdf

Randolph, M.E., Pinkerton, S.D., Bogart, L.M., Cecil, H., \& Abramson, P.R. (2007). Sexual pleasure and condom use. Archives of Sex Behavior, 36, 844-848.

Resich, M. (2007). Social justice and muliticulturalism: persistent tensions in the history of US social welfare and social work. Studies in Social Justice, 1(1), 67-92.

Reisch, M. (2008). From melting pot to multiculturalism: The impact of racial and ethnic diversity on social work and social justice in the USA. British Journal of Social Work, 38(4), 788-804. 
Reisen, C.A., Brooks, K.D., Zea, M.C., Poppen, P.J., and Bianchi, F.T. (2013). Can additive measures add to an intersectional understanding? Experiences of gay and ethnic discrimination among HIV-positive Latino gay men. Cultural Diversity and Ethnic Minority Psychology, 19(2), 208-217.

Rivera, C.P., Méndez, C.B., Gueye, N., Bachman, G.A. (2007). Family planning attitudes of medically underserved Latinas. Journal of Women's Health, 16(6), 879-882.

Robinson, B.E., Bockting, W.O., Simon Rosser, B.R., Miner, M., Coleman, E. (2002). The sexual health model: application of a sexological approach to HIV prevention. Health Education Research, 17(1), 43-57.

Rodriguez, N. (2008). Theoretical and Methodological Issues of Latina/o Research. In H.D., R.S. \& C.M. (Eds). Latinas/os in the United States: Changing the Face of America (pp. 3-15). New York, NY: Springer.

Sandfort, T.G.M., Ehrhardt, A.A. (2004). Sexual health: a useful public health paradigm or a moral imperative? Archives of Sexual Behavior, 33(3), 181-187.

Sanchez, D., Whittaker, T. A., Hamilton, E., \& Zayas, L. H. (2016). Perceived discrimination and sexual precursor behaviors in Mexican American preadolescent girls: The role of psychological distress, sexual attitudes, and marianismo beliefs. Cultural Diversity and Ethnic Minority Psychology, 22(3), 395.

Schaab, K. (2017). Locating a "better" self: aging women of color and sexual desire in Philip Kan Gotanda's the wash and Luis Santiero's our lady of the tortilla. Women's Studies, 46 (6), 574-590.

Seal, P. S., Garcés-Palacio, I. C., Halanych, J. H., \& Scarinci, I. C. (2012). Sexual health knowledge of male and female Latino immigrants. Journal of immigrant and minority health, 14(4), 673-681.

Shoveller, J.A. \& Johnson, J.L. (2006). Risky groups, risky behavior, and risky persons: dominating discourses on youth sexual health. Critical Public Health, 16(1), 4760 .

Smith, A. (2005). Beyond pro-choice versus pro-life: women of color and reproductive justice. NWSA Journal, 17(1), 119-140.

Smith, S.J. (2015). Risky sexual behavior among young adult Latinas: are acculturation and religiosity protective? Journal of Sex Research, 52(1), 43-54. 
Solorzano, D.G. \& Bernal, D.D. (2001). Examining transformational resistance through a critical race and latcrit theory and framework: Chicana and Chicano students in an urban context. Urban Education, 36(3), 308-342.

Staunaes, D. \& Sondergaard, D.M. (2011). Intersectionality: A Theoretical Adjustment. In R.B., G.G. \& N.L. Theories and Methodologies in Postgraduate Feminist Research. New York: Routledge.

Stefanic, J. (1997). Latino and Latina critical theory: an annotated bibliography. California Law Review, 85(5), 423-498.

Stephenson, K.R. \& Meston, C.M. (2015). The conditional importance of sex: exploring the association between sexual well-being and life satisfaction. Journal of Sex and Marital Therapy, 4l(1), 25-38.

Stepler, R. \& Brown, A. (2016). Hispanic Trends. Retrieved from The Pew Research Center: http://www.pewhispanic.org/2016/04/19/statistical-portrait-of-hispanicsin-the-united-states-key-charts/

Sterling, S.P. \& Sadler, L.S. (2009). Contraceptive use among adolescent Latinas living in the United States: the impact of culture and acculturation. Journal of Pediatric Health Care, 23(1), 19-28.

Sutin, A.R., Stephan, Y., \& Terracciano, A. (2016). Personality and metabolic dysfunction in young adulthood: a cross-sectional study. Journal of Health Psychology, 1-7.

Tanner, A. E., \& Hensel, D. J. (2016). Individual and Partner Attributes of Young Women's Sexual \& Protective Behaviors. Journal of Adolescent Health, 58(2), S99.

Teruya, S. A., \& Bazargan-Hejazi, S. (2013). The immigrant and Hispanic paradoxes: A systematic review of their predictions and effects. Hispanic journal of behavioral sciences, 35(4), 486-509.

Tietjen, G.E., Karmarkar, M. \& Amialchuk, A.A. (2016). Emotional abuse history and migraine among young adults: a retrospective cross-sectional analysis of the add health dataset. Headache, 57, 45-59.

Tolman, D.L. (1994). Doing desire: adolescent girls' struggles for/with sexuality. Gender and Society, 8(3), 324-342.

Tolman, D. L., \& Szalacha, L. A. (1999). Dimensions of desire. Psychology of Women Quarterly, 23(1), 7-39. 
Torres, J., Solberg, V., \& Carlstrom, A. (2002). The myth of sameness among Latino men and their machismo. American Journal of Orthopsychiatry., 72(2), 163-181.

United States Census Bureau (2016). FFF: Hispanic Heritage Month retrieved from https://census.gov/newsroom/facts-for-features/2016/cb16-ff16.html

Ulibarri, M. D., Raj, A., \& Amaro, H. (2012). Love, sex, and power revisited. HIV Prevention with Latinos: Theory, Research, and Practice.

Vargas, D.R. (2010). Representations of Latina/o sexuality in popular culture. In Asencio, M. (Ed). Latina/o Sexualities. Brunswick, NJ: Rutgers University Press.

Veenstra, G. (2011). Race, gender, class, and sexual orientation: intersecting axes of inequality and self-rated health in Canada. International journal for equity in health, 10(1), 3 .

Viruell-Fuentes, E. A., Miranda, P. Y., \& Abdulrahim, S. (2012). More than culture: structural racism, intersectionality theory, and immigrant health. Social science \& Medicine, 75(12), 2099-2106.

Ward, B.W., Dahlhamer, J.M., Galinsky, A.M. \& Joestl, S.S. (2014). Sexual orientation and health among US adults: national health interview survey 2013, Center for Disease Control, retrieved from: https://www.cdc.gov/nchs/data/nhsr/nhsr077.pdf

Warner, L.R. (2008). A best practices guide to intersectional approaches in psychological research. Sex Roles, 59, 454-463.

Warner, D. F., \& Brown, T. H. (2011). Understanding how race/ethnicity and gender define age-trajectories of disability: An intersectionality approach. Social Science \& Medicine, 72(8), 1236-1248.

Weaver, H. N. (2015). Recognizing Our Past and Moving Toward Our Future: Decolonizing Attitudes About Skin Color and Native Americans. Journal of Indigenous Social Development, 4(1), 1-15.

Widman, L., Noar, S. M., Choukas-Bradley, S., \& Francis, D. B. (2014). Adolescent sexual health communication and condom use: a meta-analysis. Health Psychology, 33(10), 1113.

Williams, D., Prior, E., \& Wegner, J. (2013). Resolving Social Problems Associated with Sexuality: Can a "Sex-Positive" Approach Help? Social Work., 58(3), 273-276.

Winter, V.R., O’Neill, E., Begun, S., Kattari, S.K., \& McKay, K. (2016). MSW student perceptions of sexual health as relevant to the profession: do social work educational experiences matter? Social Work in Health Care, 55(8), 614-634. 
World Health Organization (WHO) (1975). Education and treatment in human sexuality: the training of health professionals. Geneva, World Health Organization, 1975 (WHO Technical Report Series No. 572). Retrieved from http://www.who.int/reproductivehealth/topics/gender_rights/defining_sexual heal

World Health Organization (WHO) (2016). Gender and Human Rights, Defining Sexual Health retrieved from http://www.who.int/reproductivehealth/topics/gender rights/defining sexual heal th/en/

Wright, R. L., Bird, M., \& Frost, C. J. (2015). Reproductive health in the United States: A review of the recent social work literature. Social work, 60(4), 295-304.

Young, M., Denny, G., Luquis, R., \& Young, T. (1998). Correlates of sexual satisfaction in marriage. The Canadian Journal of Human Sexuality, 7(2), 115.

Young, M., Denny, G., Young, T., \& Luquis, R. (2000). Sexual satisfaction among married women. American Journal of Health Studies, 16(2), 73.

Zavela, P. \& Castenada, X. (2005). Sexuality and risks: gendered discourses about virginity and disease among young women of Mexican origin. Latino Studies, 3, 226-245. 